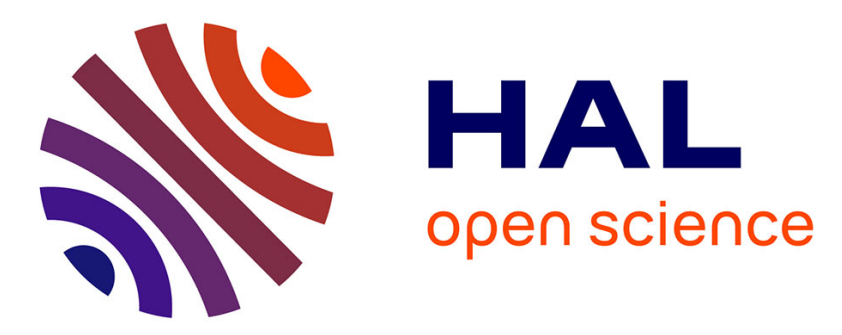

\title{
A Cavity-Shaped Diphosphane Displaying "Oschelating" Behavior
}

Rafael Gramage-Doria, Dominique Armspach, Dominique Matt, Loic Toupet

\section{To cite this version:}

Rafael Gramage-Doria, Dominique Armspach, Dominique Matt, Loic Toupet. A Cavity-Shaped Diphosphane Displaying "Oschelating" Behavior. Angewandte Chemie International Edition, 2011, 50 (7), pp.1554-1559. 10.1002/anie.201005169 . hal-01472929

HAL Id: hal-01472929

https://hal-univ-rennes1.archives-ouvertes.fr/hal-01472929

Submitted on 30 Nov 2020

HAL is a multi-disciplinary open access archive for the deposit and dissemination of scientific research documents, whether they are published or not. The documents may come from teaching and research institutions in France or abroad, or from public or private research centers.
L'archive ouverte pluridisciplinaire HAL, est destinée au dépôt et à la diffusion de documents scientifiques de niveau recherche, publiés ou non, émanant des établissements d'enseignement et de recherche français ou étrangers, des laboratoires publics ou privés. 


\section{A cavity-shaped diphosphane displaying os-chelating behaviour}

Rafael Gramage-Doria, Dominique Armspach, * Dominique Matt, * and Loic Toupet

[*] R. Gramage-Doria, Prof. D. Armspach, Dr. D. Matt Laboratoire de Chimie Inorganique Moléculaire et Catalyse, Institut de Chimie UMR 7177 CNRS

Université de Strasbourg 67008 Strasbourg Cedex (France)

Dr. L. Toupet Groupe Matière Condensée et Matériaux, UMR 6626 CNRS Université de Rennes 1 35042 Rennes Cedex (France)

[**] This work was supported by the CNRS and the Région Alsace (grant to RGD) 
Molecules that combine the properties of a transition metal with those of an appended cavity have attracted a great deal of attention. ${ }^{[1-3]}$ So far, research in this area has focused on four main objectives: a) the design and synthesis of systems exploiting the binding properties of a receptor unit linked to a transition metal centre, with the aim of producing catalysts that mimic an enzyme ${ }^{[4-8]}$ b) the study of metal-centered reactions taking place in a confined environment thereby favouring highly regio-, stereo- and shape-selective reactions, ${ }^{[9-13]} \mathrm{c}$ ) the metal-assisted entrapment and recognition of ionic species; ${ }^{[14-16]} d$ ) the construction of sensors capitalising on a electro- or photoactive metal-unit covalently attached to a close, cavityshaped receptor. ${ }^{[17,18]}$

While the coordination chemistry of many multitopic ligands giving rise to 3-D architectures such as capsules, cages, bowls and boxes has been thoroughly investigated, ${ }^{[19-25]}$ the use of such ligands for generating oscillatory motion about a metal metal ion has not been considered yet, although examples of transition metals moving around the periphery of such an object are known. ${ }^{[26]}$ Herein we show how a cavity bearing two introverted donor atoms may behave as a balance wheel swinging about a central metal unit. Our approach is based on the use of a rigid, cyclodextrin-based diphosphane characterised by a long P...P separation that results in highly unsymmetrical chelation.

Diphosphine 6 was obtained in $20 \%$ overall yield according to Scheme 1 . Its synthesis began with a regioselective double capping of native $\beta$-cyclodextrin $(\beta$-CD) using the bulky dialkylating reagent $\mathbf{1}^{[27]}$ The non-alkylated hydroxyl groups were subsequently methylated with $\mathrm{NaH} / \mathrm{MeI}$, resulting in the $A B D E$-functionalised intermediate 2 (yield $50 \%$ !). Deprotection with $\mathrm{HBF}_{4}$, leading to tetrol $\mathbf{3}$, followed by reaction with mesyl chloride in pyridine afforded tetramesylate 4 . Reaction of the latter with $\mathrm{Li}_{2} \mathrm{PPh}$ in THF gave diphosphine 6 in $c a$. 70\% yield beside two other, unidentified products. Work-up required the preparation of the diborane adduct 5, which was separated chromatographically. Finally, $\mathbf{5}$ was treated with $\mathrm{HNEt}_{2}$ to afford quantitatively 6. As expected, the ${ }^{31} \mathrm{P}$ NMR spectrum of 6 in $\mathrm{C}_{6} \mathrm{D}_{6}$ shows two very near singlets, seen at $\delta=-15.0$ and $-15.2 \mathrm{ppm}$, respectively (in $\mathrm{CDCl}_{3}$ the spectrum showed but a unique peak). Prolonged standing in air of a solution of 6 in $\mathrm{MeOH}$ produced the di(phosphine oxide) 7, the structure of which was determined by an X-ray diffraction study (Fig. 1). In the solid state, the two $\mathrm{P}=\mathrm{O}$ vectors of 7 point towards the interior of the cavity, the P...P separation being $6.91 \AA$. Two non-bridged glucose units are tipped towards the $\mathrm{CD}$ axis, reflecting some strain within the CD.

Despite the long separation between the two phosphorus atoms, diphosphine 6 turned out to be suitable for chelation. Thus, for example, reaction with $[\mathrm{Au}(\mathrm{tht})(\mathrm{thf})] \mathrm{PF}_{6}($ tht $=$ 
tetrahydrothiophene; THF = tetrahydrofuran) led quantitatively to complex $\mathbf{8}$. The mass spectrum of 8 showed an intense peak at $m / z=1717.62$ having exactly the isotopic profile expected for $[\mathrm{Au} \bullet 6]^{+}$. Furthermore, the ${ }^{31} \mathrm{P}$ NMR $\left(\mathrm{CD}_{2} \mathrm{Cl}_{2},{ }^{2} 5^{\circ} \mathrm{C}\right)$ spectrum displayed an $\mathrm{AB}$ pattern with a $J(\mathrm{PP})$ coupling constant of $326 \mathrm{~Hz}$, a value which is in accord with a very large bite angle. Molecular models indicate that the bite angle is close to $160^{\circ}$. The good chelating properties of 6 were further

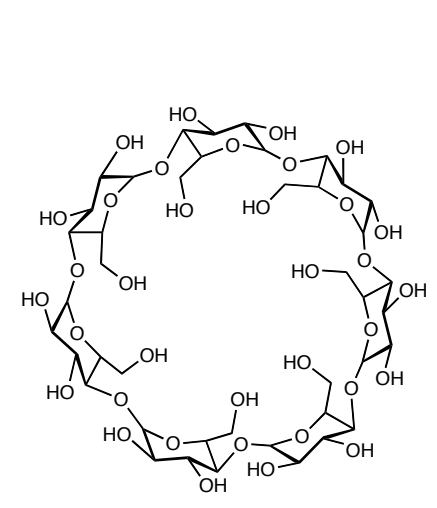

$\beta-\mathrm{CD}$

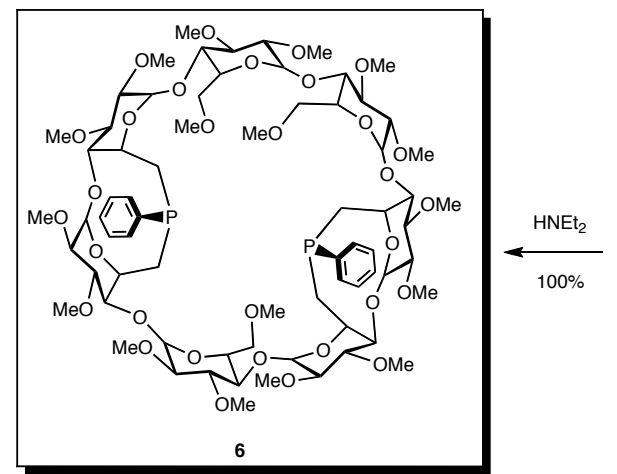

Scheme 1. Stepwise buildup of 6

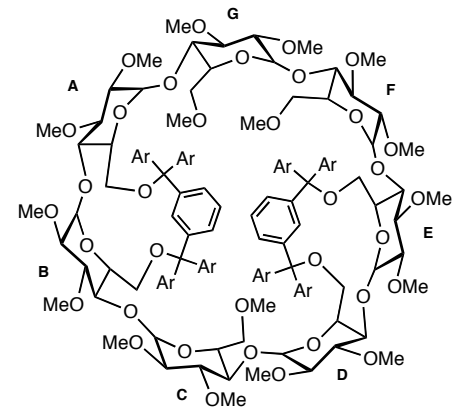

$2 \mathrm{Ar}=p-\mathrm{BBu}^{-} \mathrm{C}_{6} \mathrm{H}_{4}$

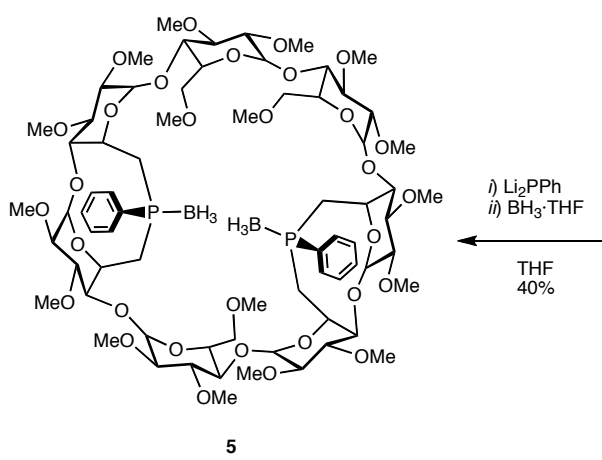

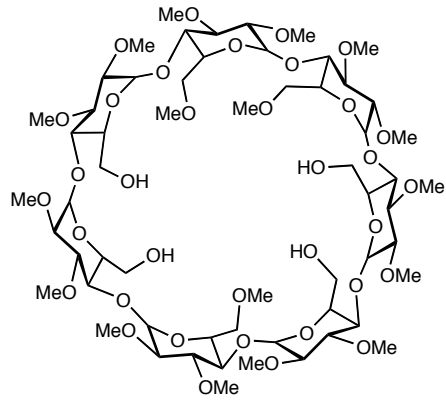

3
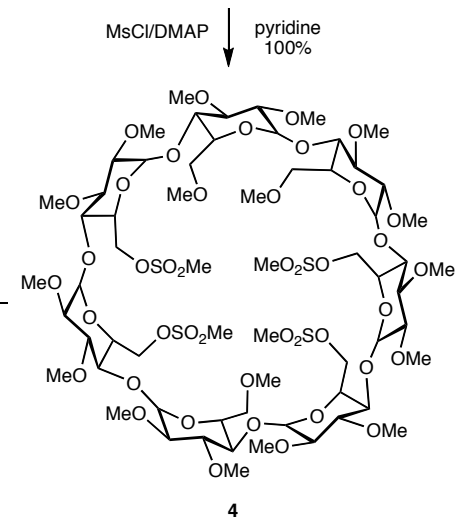


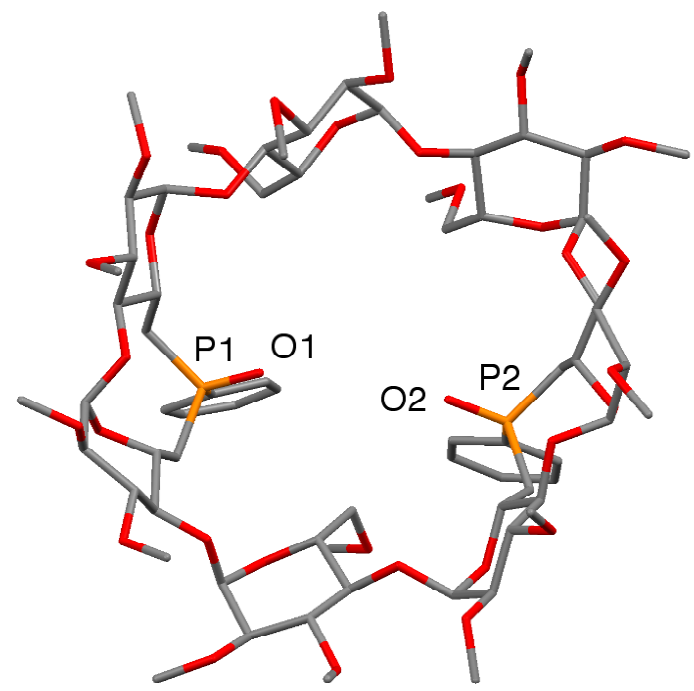

Figure 1. X-ray structure of the di(phosphine oxide) 7 (view from the secondary face). Solvent molecules have been omitted. Important distances $(\AA ̊)$ : P1-P2 6.91; O1-O2 4.32.

confirmed by its reaction with $\left[\mathrm{PdCl}_{2}(\mathrm{PhCN})_{2}\right],\left[\mathrm{PtCl}_{2}(\mathrm{PhCN})_{2}\right]$, and $\left[\mathrm{RhCl}(\mathrm{CO})_{2}\right]_{2}$ leading to the chelate complexes 9-11, respectively, in 100\% yield (Scheme 2 and SI). As in 8, the corresponding large $J\left(\mathrm{PP}^{\prime}\right)$ coupling constants (see $\mathrm{SI}$ ) reflect the large bite angle of the ligand. ${ }^{[28,29]}$ 


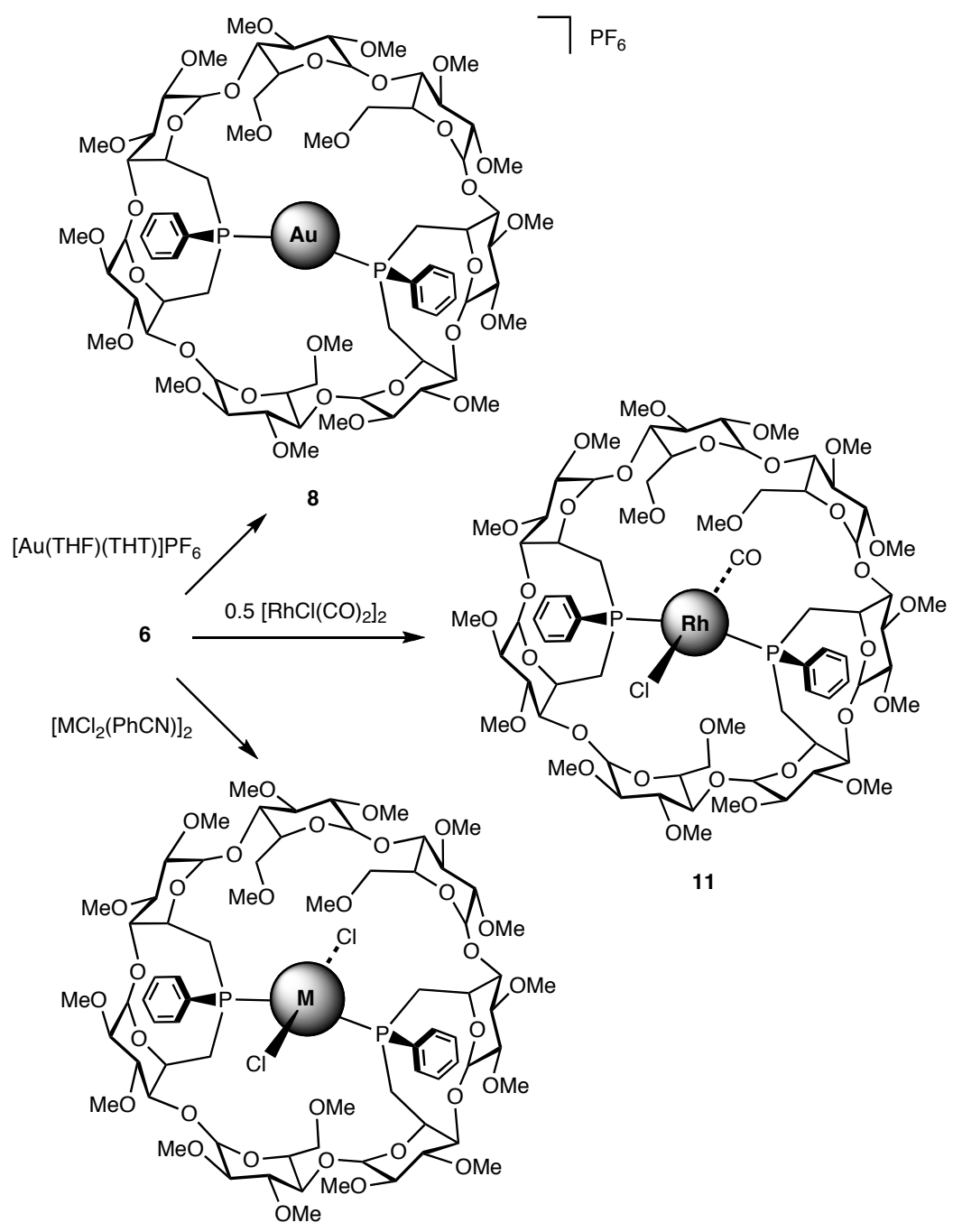

$\begin{aligned} 9 M & =P d \\ 10 M & =P t\end{aligned}$

Scheme 2. Synthesis of complexes 8-11.

As shown by a variable temperature NMR study, complex 10 displays fluxional behaviour in solution. The ${ }^{31} \mathrm{P}$ NMR $\left(\mathrm{CD}_{2} \mathrm{Cl}_{2}\right)$ spectrum of 10 , measured at $-80^{\circ} \mathrm{C}$, revealed the presence of two species (10a and 10b) present in a 1:1 ratio, each caracterised by an $\mathrm{ABX}$ pattern $\left({ }^{2} J(\mathrm{AB})\right.$ $=492$ and $476 \mathrm{~Hz}$, respectively; ${ }^{1} J(\mathrm{PPt})$ coupling poorly resolved) (Fig. 2, bottom). Upon raising the temperature, the signals first broadened, then coalesced near $-15^{\circ} \mathrm{C}$, and finally merged into a single $\mathrm{ABX}$ spectrum $\left({ }^{2} J(\mathrm{AB})=496 \mathrm{~Hz},{ }^{1} J(\mathrm{PPt})=2510 \mathrm{~Hz}\right)$ (Fig. 2, top). The observed data are consistent with exchange between two complexes both of which contain a close to linear P-Pt-P unit. A variable temperature ${ }^{1} \mathrm{H}$ NMR study was also carried out which confirmed the 1:1 stoichiometry of the equilibrating species. Both series of experiments led to a free energy of activation $\Delta \mathrm{G}^{\neq}=11.3 \pm 0.2 \mathrm{kcal} \mathrm{mol}^{-1}$. Interestingly, the low temperature ${ }^{31} \mathrm{P}$ 
NMR spectra revealed two AB patterns with a large separation between the A and B parts ( $c a$. $12 \mathrm{ppm}$ ), indicating that the two $\mathrm{P}$ atoms of each complex are coordinated to the platinum atom with unequal strength. Note that one of the phosphorus signals appears near the midpoint between the signal of the free ligand and that of the other signal. As shown by an off-resonance ${ }^{31} \mathrm{P}\left\{{ }^{1} \mathrm{H}\right\}-{ }^{31} \mathrm{P}\left\{{ }^{1} \mathrm{H}\right\}$ ROESY NMR experiment (SI), the "strongly" coordinated phosphorus atom of each species is in exchange with the "weakly" coordinated phosphorus atom of the other isomer.

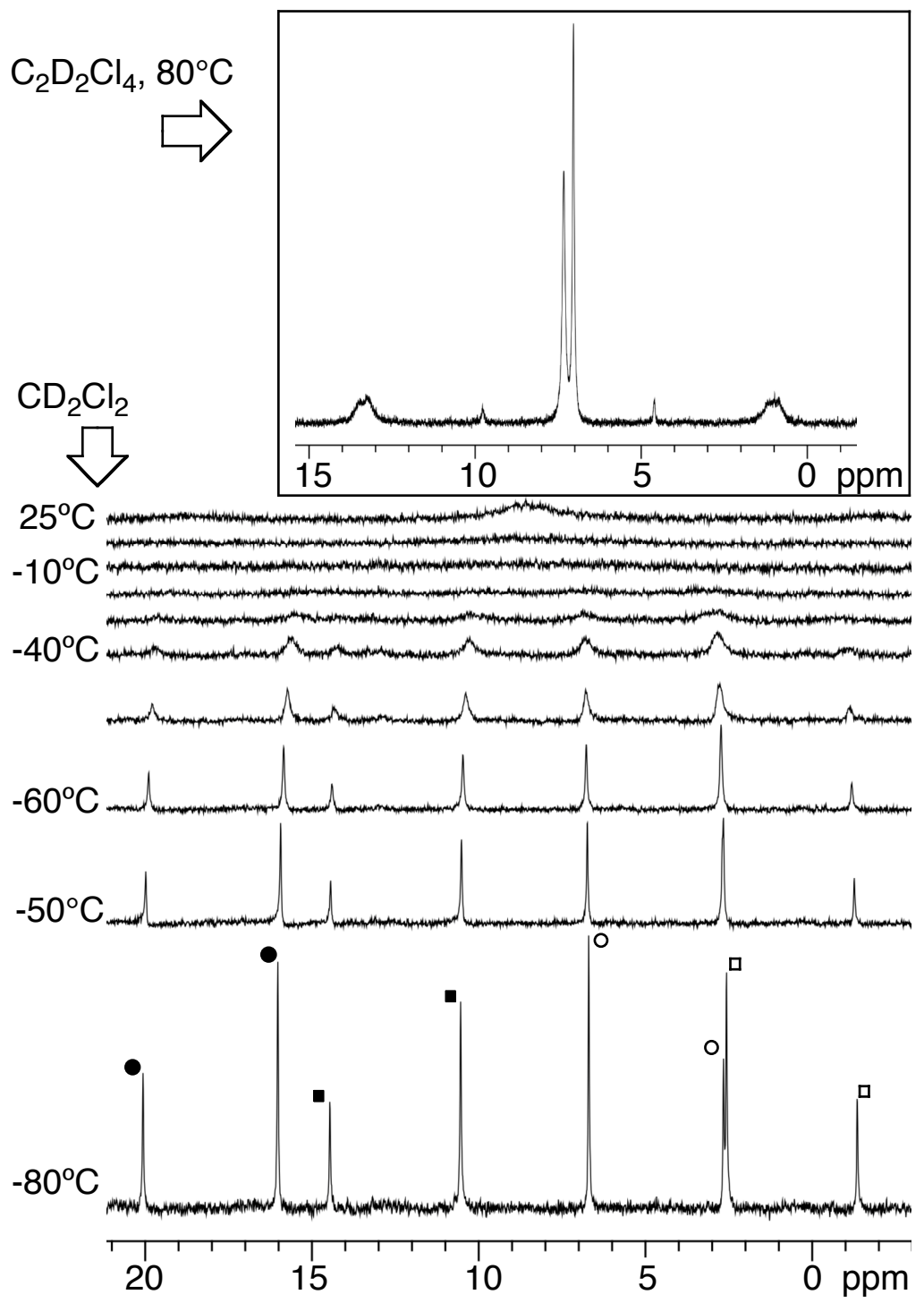

Figure 2. ${ }^{31} \mathrm{P}\left\{{ }^{1} \mathrm{H}\right\}$-NMR variable temperature study of the platinum complex 10. The $\mathrm{AB}$ patterns of the two equilibrating species are represented by dots and squares. Filled symbols are for the A parts, open symbols for the B parts. 

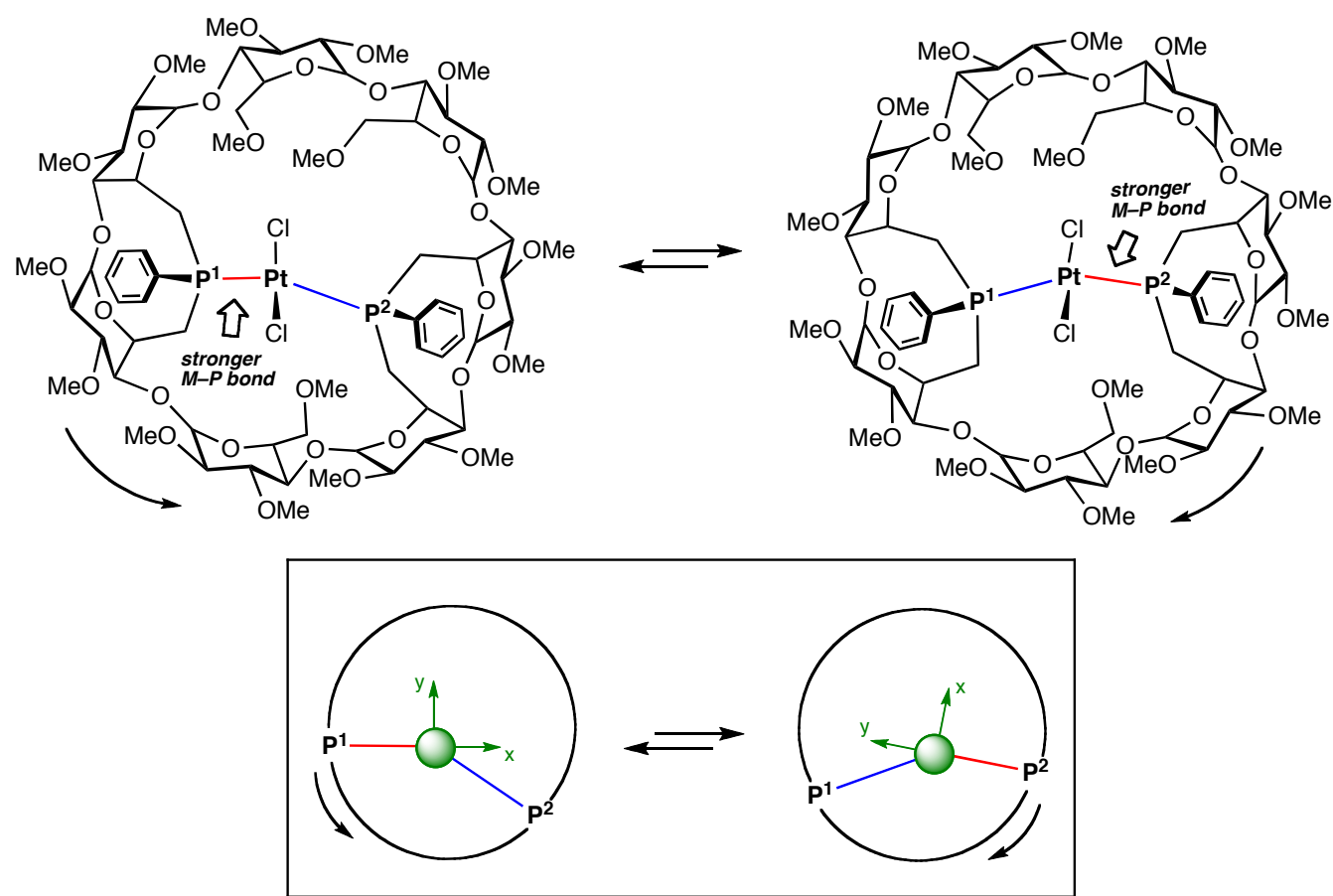

Scheme 3. Balance wheel movement of ligand 6 in complex 10 (view along the CD axis). The green arrows in the lower part indicate the orientations of the $d_{x 2-y 2}$ orbital involved in formation of the two MP bonds.

Overall, the best way to describe these findings is to consider that the $d_{x 2-y 2}$ orbital involved in formation of the M-P bonds changes its orientation in a pendular fashion so as to adopt in alternation different overlaps with each of the two convergent, but non-aligned phosphorus lone pairs. Consequently, metal binding to the two phosphorus donors is inequivalent in a given species (Scheme 3). The observed isomerisation is probably also accompanied by a slight displacement of the metal centre. Thus, being unable to form an authentic transcomplex (i.e. with a $\mathrm{P}-\mathrm{M}-\mathrm{P}$ angle of $180^{\circ}$ ), diphosphane 6 may be regarded as a frustrated chelator, which compensates the metal electron deficiency generated at one coordination site by oscillating about the complexed metal ion. We propose to term this type of bidentate ligand an os-chelating (contraction of "oscillating" and "chelating") species. It is worth mentioning here that the observed fluxionality is different from that found in complexes containing hemilabile ligands, the latter leading to intermediates in which one end is totally dissociated. ${ }^{[30-33]}$ It should be emphasized that similar dynamics were observed for all three complexes 8, 9 and 11. Furthermore, for all four complexes investigated the $J\left(\mathrm{PP}^{\prime}\right)$ coupling constants persisted over the temperature range $-80^{\circ} \mathrm{C} /+80^{\circ} \mathrm{C}$. In other words, the observed 
dynamic behaviour occurs without dissociation of the $\mathrm{M}-\mathrm{P}$ bonds. This phenomenon may be regarded as a variant of bond-stretch isomerism. ${ }^{[34,35]}$

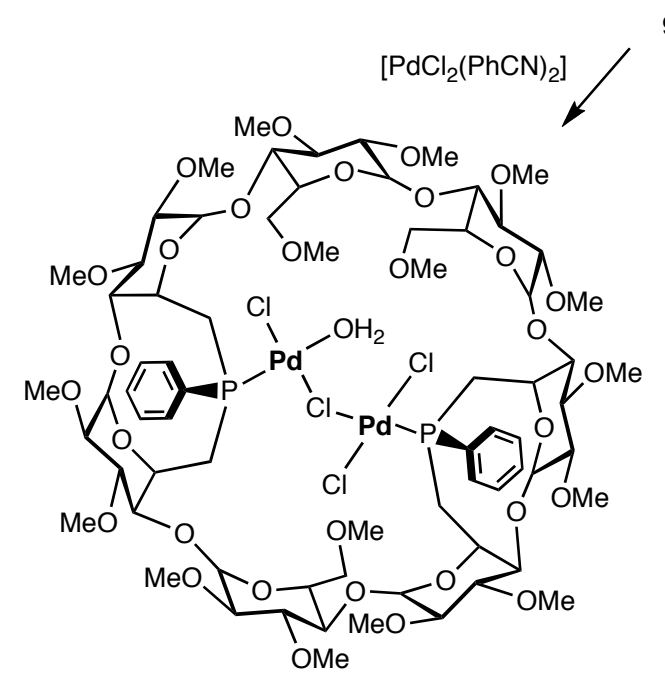

12

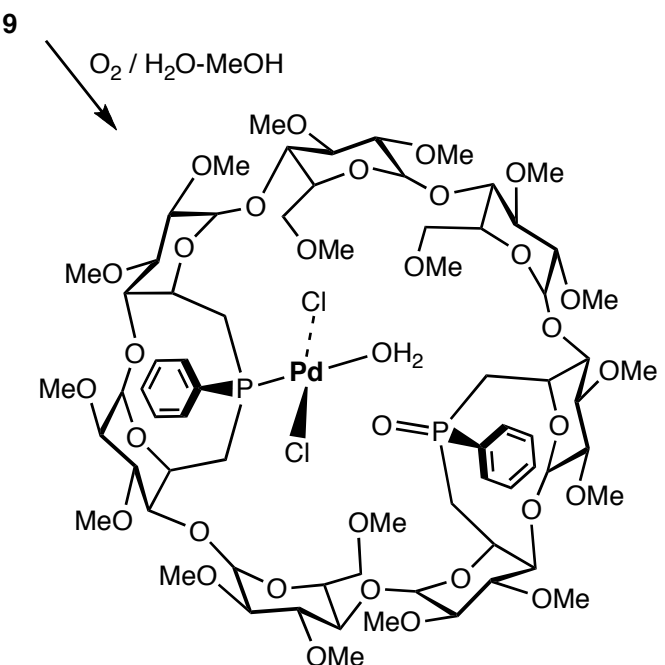

13

Scheme 4. Exploiting the os-chelating property of 6

We anticipated that the dynamic nature of $\mathbf{6}$ in the chelate complexes would weaken the $\mathrm{P}-\mathrm{M}$ bonds. In fact, treatment of 9 with $\left[\mathrm{PdCl}_{2}(\mathrm{PhCN})_{2}\right]$ led formally to insertion of a $\mathrm{PdCl}_{2}$ fragment into one of the $\mathrm{Pd}-\mathrm{P}$ bonds of 9 . The resulting complex, 12, crystallised with a water molecule coordinated to one of the palladium atoms (Scheme 4, Fig. 3). It is likely that owing to its rigidity, diphosphine 6 cannot adapt to a $\mathrm{Pd}_{2} \mathrm{Cl}_{2}(\mu-\mathrm{Cl})_{2}$ fragment having the usual flat or roof structure and therefore prefers to cap a $\mathrm{Pd}_{2} \mathrm{Cl}_{4}$ unit having only a single chlorido bridge. Another reaction directly related to the os-chelating behaviour of 6 is the reaction between 9 and $\mathrm{O}_{2} / \mathrm{H}_{2} \mathrm{O}$ in methanol, giving 13 , seemingly formed by cleavage of one of the Pd-P bonds and its substitution by a bond to $\mathrm{H}_{2} \mathrm{O}$, followed by oxidation of the dissociated P atom. ${ }^{[36]}$ The P...P' separation in 13 (6.6 ̊) 

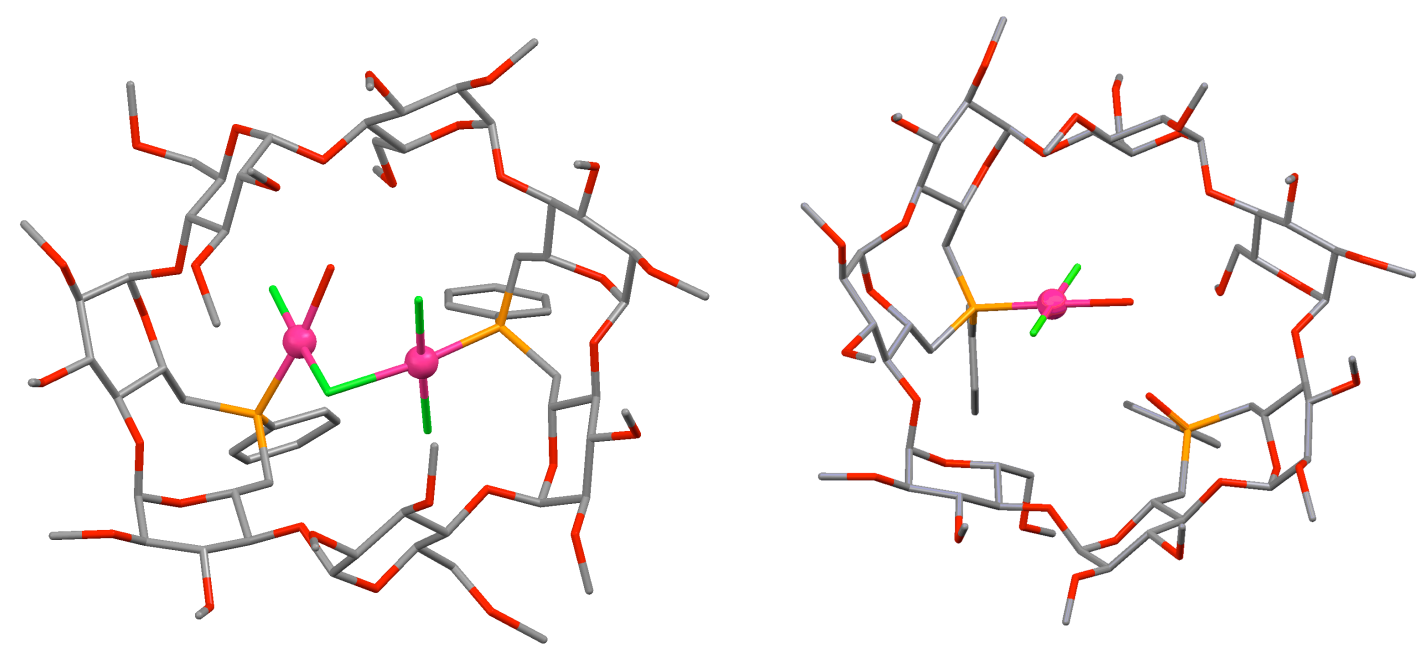

Figure 3. X-ray structures of complex 12 (left) and the monophosphine-Pd complex 13 (right)

is close to the one found in 7 . We note that both complexes, 12 and 13 , contain a "(phosphine) $\mathrm{PdX}_{2}\left(\mathrm{H}_{2} \mathrm{O}\right)$ " unit, thus constituting rare examples of [LL'PdX $\mathrm{L}_{2}$ complexes containing a single phosphine ligand. Overall, the coordinative properties of $\mathbf{6}$ are markedly different from those of the previously reported $\alpha$-CD analogue (TRANSDIP). ${ }^{[37]}$

In conclusion, using a capping methodology that relies on the use of the bulky dialkylating agent $\mathbf{1}$, we have synthesised the first large bite angle diphosphine (6) based on a $\beta$-CD backbone. Owing to the rigidity of this ligand as well as the large separation between the phosphorus atoms, 6 behaves towards transition metal ions in an unsymmetrical chelator, inducing rapid oscillation of the chelate about the coordinated metal centre. This phenomenon occurs without dissociation of the phosphorus atoms. The unprecedented chelating behaviour of 6 enables the formation of monophosphine complexes located inside a CD, thereby opening a way to the further study of organometallic catalysts operating in a confined environment.

\section{Experimental Section}

Full experimental details including X-ray structural data are given in the Supporting Information. CCDC-756653, 758028 and 770728 contain the supplementary crystallographic data for 7, 12 and, 13, respectively. These data can be obtained free of charge from the Cambridge Crystallographic Data Centre via www.ccdc.cam.ac.uk/data_request/cif. 
6: ${ }^{1} \mathrm{H}$ NMR $\left(300.1 \mathrm{MHz}, \mathrm{CDCl}_{3}, 25^{\circ} \mathrm{C}\right): \delta$ (assignment by COSY) $=1.73(\mathrm{~m}, 2 \mathrm{H}, \mathrm{H}-$ $\left.6 \mathrm{a}^{\mathrm{A}, \mathrm{D} \text { or B,E}}\right), 1.87\left(\mathrm{~m}, 2 \mathrm{H}, \mathrm{H}-6 \mathrm{a}^{\mathrm{B}, \mathrm{E}}\right.$ or A,D $), 2.81\left(\mathrm{td}, 2 \mathrm{H},{ }^{2} J_{\mathrm{H}-6 \mathrm{~b}, \mathrm{P}}={ }^{2} J_{\mathrm{H}-6 \mathrm{~b}, \mathrm{H}-6 \mathrm{a}}=13.5 \mathrm{~Hz},{ }^{3} J_{\mathrm{H}-6 \mathrm{~b}, \mathrm{H}-5}\right.$ $\left.=3.9 \mathrm{~Hz}, \mathrm{H}-6 \mathrm{~b}^{\mathrm{A}, \mathrm{D} \text { or B,E}}\right), 3.05-3.29\left(12 \mathrm{H}, \mathrm{H}-2, \mathrm{H}-6 \mathrm{a}^{\mathrm{C}, \mathrm{F}, \mathrm{G}}, \mathrm{H}-6 \mathrm{~b}^{\mathrm{B}, \mathrm{E}}\right.$ or A,D $), 3.11(\mathrm{~s}, 3 \mathrm{H}, \mathrm{OMe})$, 3.20 (s, 3 H, OMe), 3.27 (s, 3 H, OMe), 3.30-3.72 (14 H, H-3, H-4), 3.47 (s, 3 H, OMe), 3.48 (s, $3 \mathrm{H}, \mathrm{OMe}), 3.49$ (s, $3 \mathrm{H}, \mathrm{OMe}), 3.50(\mathrm{~s}, 6 \mathrm{H}, \mathrm{OMe}), 3.55$ (s, $6 \mathrm{H}, \mathrm{OMe}), 3.60$ (s, $3 \mathrm{H}$, OMe), 3.62 (s, $3 \mathrm{H}, \mathrm{OMe}), 3.65$ (s, $9 \mathrm{H}, \mathrm{OMe}), 3.66$ (s, $6 \mathrm{H}, \mathrm{OMe}), 3.81-3.42\left(8 \mathrm{H}, \mathrm{H}-5^{\mathrm{A}, \mathrm{D} \text { or }}\right.$ $\left.{ }^{\mathrm{B}, \mathrm{E}}, \mathrm{H}-5^{\mathrm{C}, \mathrm{F}, \mathrm{G}}, \mathrm{H}-6 \mathrm{~b}^{\mathrm{C}, \mathrm{F}, \mathrm{G}}\right), 4.30\left(\mathrm{~m}, 2 \mathrm{H}, \mathrm{H}-5^{\mathrm{B}, \mathrm{E} \text { or A,D }}\right), 4.95-5.05(6 \mathrm{H}, \mathrm{H}-1), 5.22\left(\mathrm{~d}, 1 \mathrm{H},{ }^{3} J_{\mathrm{H}-1, \mathrm{H}-}\right.$ $\left.{ }_{2}=3.7 \mathrm{~Hz}, \mathrm{H}-1\right), 7.20-7.28(6 \mathrm{H}, m-\mathrm{H}, p-\mathrm{H}), 7.41-7.51(4 \mathrm{H}, o-\mathrm{H}) \mathrm{ppm} ;{ }^{13} \mathrm{C}\left\{{ }^{1} \mathrm{H}\right\} \mathrm{NMR}$ $\left(125.8 \mathrm{MHz} \mathrm{C}_{6} \mathrm{D}_{6}, 25^{\circ} \mathrm{C}\right): \delta$ (assignment by HMQC) $=28.66\left(\mathrm{~d},{ }^{1} J_{\mathrm{C}, \mathrm{P}}=15.4 \mathrm{~Hz}\right), 28.69(\mathrm{~d}$, $\left.{ }^{1} J_{\mathrm{C}, \mathrm{P}}=16.2 \mathrm{~Hz}\right)\left(\mathrm{C}-6^{\mathrm{A}, \mathrm{D} \text { or B,E}}\right), 34.99\left(\mathrm{~d},{ }^{1} J_{\mathrm{C}, \mathrm{P}}=19.1 \mathrm{~Hz}\right), 35.06\left(\mathrm{~d},{ }^{1} J_{\mathrm{C}, \mathrm{P}}=19.7 \mathrm{~Hz}\right)\left(\mathrm{C}-66^{\mathrm{B}, \mathrm{E} \text { or }}\right.$ A,D), $58.14[\times 2], 58.20,58.32,58.71,59.10,59.14,59.44,59.68,59.74,61.77,61.79,61.90$, $62.00,62.05,62.25[\times 2](\mathrm{OMe}), 67.54\left(\mathrm{~d},{ }^{2} J_{\mathrm{C}, \mathrm{P}}=11.7 \mathrm{~Hz}, \mathrm{C}-5^{\mathrm{A} \text { or D}}\right), 67.57\left(\mathrm{~d},{ }^{2} J_{\mathrm{C}, \mathrm{P}}=11.7 \mathrm{~Hz}\right.$, $\left.\mathrm{C}-5^{\mathrm{D} \text { or A}}\right), 71.53[\times 2], 71.80\left(\mathrm{C}-5^{\mathrm{C}, \mathrm{F}, \mathrm{G}}\right), 72.13\left(\mathrm{~d},{ }^{\mathrm{TS}} J_{\mathrm{C}, \mathrm{P}}=2.6 \mathrm{~Hz}, \mathrm{C}-6^{\mathrm{C}}\right), 72.39[\times 2]\left(\mathrm{C}-6^{\mathrm{F}, \mathrm{G}}\right)$, $73.95\left(\mathrm{~d},{ }^{2} J_{\mathrm{C}, \mathrm{P}}=14.3 \mathrm{~Hz}, \mathrm{C}-5^{\mathrm{B} \text { or E}}\right), 74.26\left(\mathrm{~d},{ }^{2} J_{\mathrm{C}, \mathrm{P}}=13.6 \mathrm{~Hz}, \mathrm{C}-5^{\mathrm{E}}\right.$ or B $), 79.75,82.22,82.31$, $82.37,82.42,82.97,83.15,83.23,83.36$ [×3], 83.42, 83.46, 83.58, 84.07, 84.55, 84.70 (C-2, $\left.\mathrm{C}-3, \mathrm{C}-4^{\mathrm{C}, \mathrm{F}, \mathrm{G}}\right), 87.15\left(\mathrm{~d},{ }^{3} J_{\mathrm{C}, \mathrm{P}}=8.0 \mathrm{~Hz}, \mathrm{C}-4^{\mathrm{B} \text { or E}}\right), 87.32\left(\mathrm{~d},{ }^{3} J_{\mathrm{C}, \mathrm{P}}=8.0 \mathrm{~Hz}, \mathrm{C}-4^{\mathrm{E}}\right.$ or B$), 90.23(\mathrm{~d}$, $\left.{ }^{3} J_{\mathrm{C}, \mathrm{P}}=2.7 \mathrm{~Hz}, \mathrm{C}-4^{\mathrm{A} \text { or D }}\right), 90.38\left(\mathrm{~d},{ }^{3} J_{\mathrm{C}, \mathrm{P}}=2.6 \mathrm{~Hz}, \mathrm{C}-4^{\mathrm{D} \text { or A }}\right), 99.27,99.32,99.69,99.90,99.96$ [×2], $101.19(\mathrm{C}-1), 128.96,129.03(p-\mathrm{C}), 129.15$ [×2] (overlapping d, $\left.{ }^{3} J_{\mathrm{C}, \mathrm{P}}=4.8 \mathrm{~Hz}, m-\mathrm{C}\right)$, $132.47\left(\mathrm{~d},{ }^{2} J_{\mathrm{C}, \mathrm{P}}=18.7 \mathrm{~Hz}, o-\mathrm{C}\right), 132.51\left(\mathrm{~d},{ }^{2} J_{\mathrm{C}, \mathrm{P}}=18.7 \mathrm{~Hz}, o-\mathrm{C}\right), 142.33[\times 2]\left(\mathrm{d},{ }^{1} J_{\mathrm{C}, \mathrm{P}}=11.7\right.$ $\mathrm{Hz}$, ipso-C) ppm; ${ }^{31} \mathrm{P}\left\{{ }^{1} \mathrm{H}\right\} \mathrm{NMR}\left(202.5 \mathrm{MHz} \mathrm{C}_{6} \mathrm{D}_{6}, 25^{\circ} \mathrm{C}\right): \delta=-15.0$ (s), -15.2 (s) ppm; elemental analysis (\%) calcd for $\mathrm{C}_{71} \mathrm{H}_{110} \mathrm{O}_{31} \mathrm{P}_{2} \cdot 0.5 \mathrm{CH}_{2} \mathrm{Cl}_{2}(1521.56+42.47)$ : $\mathrm{C} 54.91, \mathrm{H} 7.15$, found: C 54.82, H 7.35; MS (ESI-TOF): $m / z(\%): 1521.57(100)[M+\mathrm{H}]^{+}$.

8: ${ }^{31} \mathrm{P}\left\{{ }^{1} \mathrm{H}\right\}$ NMR $\left(121.5 \mathrm{MHz}, \mathrm{CD}_{2} \mathrm{Cl}_{2},-80^{\circ} \mathrm{C}\right): \delta=40.6$ and 31.6 (AB system, ${ }^{2} J_{\mathrm{P} 1, \mathrm{P} 2}=$ $326 \mathrm{~Hz}$ ), 38.0 and 33.8 (AB system, ${ }^{2} J_{\mathrm{P} 1, \mathrm{P} 2}=326 \mathrm{~Hz}$ ), -144.3 (hept, ${ }^{1} J_{\mathrm{P}, \mathrm{F}}=716 \mathrm{~Hz}$ ) ppm.

9: ${ }^{31} \mathrm{P}\left\{{ }^{1} \mathrm{H}\right\}$ NMR $\left(121.5 \mathrm{MHz}, \mathrm{CD}_{2} \mathrm{Cl}_{2},-80^{\circ} \mathrm{C}\right)=21.7$ and $11.4(2 \mathrm{~d}, \mathrm{AB}$ system, $\left.{ }^{2} J_{\mathrm{P} 1, \mathrm{P} 2}=564 \mathrm{~Hz}\right), 17.7$ and $5.6\left(2 \mathrm{~d}, \mathrm{AB}\right.$ system, $\left.{ }^{2} J_{\mathrm{P} 1, \mathrm{P} 2}=549 \mathrm{~Hz}\right) \mathrm{ppm}$.

10: ${ }^{31} \mathrm{P}\left\{{ }^{1} \mathrm{H}\right\}$ NMR $\left(121.5 \mathrm{MHz}, \mathrm{CDCl}_{3}, 25^{\circ} \mathrm{C}\right): \delta=8.9$ (br s with br Pt satellites, ${ }^{1} J_{\mathrm{P}, \mathrm{Pt}} \approx$ $2500 \mathrm{~Hz}) \mathrm{ppm} ;{ }^{31} \mathrm{P}\left\{{ }^{1} \mathrm{H}\right\} \mathrm{NMR}\left(121.5 \mathrm{MHz}, \mathrm{CD}_{2} \mathrm{Cl}_{2},-80^{\circ} \mathrm{C}\right)=0.97$ and $12.20(2 \mathrm{~d}, \mathrm{AB}$ system, $\left.{ }^{2} J_{\mathrm{P} 1, \mathrm{P} 2}=476 \mathrm{~Hz}\right), 5.02$ and $17.76\left(2 \mathrm{~d}, \mathrm{AB}\right.$ system, $\left.{ }^{2} J_{\mathrm{P} 1, \mathrm{P} 2}=492 \mathrm{~Hz}\right) \mathrm{ppm}\left({ }^{1} J_{\mathrm{Pt}, \mathrm{P}}\right.$ poorly resolved); ${ }^{31} \mathrm{P}\left\{{ }^{1} \mathrm{H}\right\}$ NMR $\left(202.5 \mathrm{MHz}, \mathrm{C}_{2} \mathrm{D}_{2} \mathrm{Cl}_{4},+80^{\circ} \mathrm{C}\right)=7.04$ and $7.35(2 \mathrm{~d}$ with $\mathrm{Pt}$ satellites, ABX system, $\left.{ }^{1} J_{\mathrm{Pt}, \mathrm{P} 1}=2507 \mathrm{~Hz},{ }^{1} J_{\mathrm{Pt}, \mathrm{P} 2}=2481 \mathrm{~Hz},{ }^{2} J_{\mathrm{P} 1, \mathrm{P} 2}=496 \mathrm{~Hz}\right) \mathrm{ppm}$. 
11: ${ }^{31} \mathrm{P}\left\{{ }^{1} \mathrm{H}\right\}$ NMR $\left(162.0 \mathrm{MHz}, \mathrm{CD}_{2} \mathrm{Cl}_{2},-80^{\circ} \mathrm{C}\right)=28.0$ and $9.4(2 \mathrm{dd}, \mathrm{ABX}$ system, ${ }^{2} J_{\mathrm{P} 1, \mathrm{P} 2}=354 \mathrm{~Hz},{ }^{1} J_{\mathrm{P}, \mathrm{Rh}}=118$ and 118$), 16.4$ and $9.4\left(2 \mathrm{dd}, \mathrm{ABX}\right.$ system, ${ }^{2} J_{\mathrm{P} 1, \mathrm{P} 2}=354 \mathrm{~Hz}$, ${ }^{1} J_{\mathrm{P}, \mathrm{Rh}}=118$ and 118$) \mathrm{ppm}$

Keywords: metallocavitands - cyclodextrins $\cdot$ trans chelating diphosphane $\cdot$ molecular dynamics $\bullet$ transition metal chemistry $\bullet$

[1] B. Kersting, U. Lehmann, 2009, Chemistry of Metalated Container Molecules, in Advances in Inorganic Chemistry (Eds. R. van Eldik and C. D. Hubbard), Vol 61, The Netherlands: Elsevier, 2009, pp. 407-470.

[2] C. Jeunesse, D. Armspach, D. Matt, Chem. Commun. 2005, 5603-5614.

[3] P. Ballester, A. Vidal-Ferran, in Supramolecular Catalysis (Ed. P. W. N. M. van Leeuwen), Wiley-VCH, Weinheim, 2008, pp 1-27.

[4] R. Breslow, S. D. Dong, Chem. Rev. 1998, 98, 1997-2011.

[5] M. T. Reetz, S. R. Waldvogel, Angew. Chem., Int. Ed. Engl. 1997, 36, 865-867.

[6] F. Hapiot, S. Tilloy, E. Monflier, Chem. Rev. 2006, 106, 767-781.

[7] N. Le Poul, B. Douziech, J. Zeitouny, G. Thiabaud, H. Colas, F. Conan, N. Cosquer, Y. Jabin, C. Lagrost, P. Hapiot, O. Reinaud, Y. Le Mest*, J. Am. Chem. Soc. 2009, 131, 17800-17807.

[8] L. Monnereau, D. Sémeril, D. Matt, L. Toupet, Chem. Eur. J. 2010, 16, 9237-9247.

[9] J. A. A. W. Elemans, E. J. A. Bijsterveld, A. E. Rowan, R. J. M. Nolte, Chem. Comm. 2000, 2443-2444.

[10] G. Steinfeld, V. Lozan, B. Kersting, Angew. Chem. Int. Ed. Engl. 2003, 42, 22612263.

[11] D. Sémeril, C. Jeunesse, D. Matt, L. Toupet, Angew. Chem. Int. Ed. Engl. 2006, 58105814.

[12] A. B. C. Deutman, C. Monnereau, J. A. A. W. Elemans, G. Ercolani, R. J. M. Nolte, A. E. Rowan, Science 2008, 322, 1668-1671.

[13] T. S. Koblenz, J. Wassenaar, J. N. H. Reek, Chem. Soc. Rev. 2008, 37, 247-262.

[14] M. Staffilani, K. S. B. Hancock, J. W. Steed, K. T. Holman, J. L. Atwood, R. K. Juneja, R. S. Burkhalter, J. Am. Chem. Soc. 1997, 119, 6324-6335.

[15] F. Fochi, P. Jacopozzi, E. Wegelius, K. Rissanen, P. Cozzini, E. Marastoni, E. Fisicaro, P. Manini, R. Fokkens, E. Dalcanale, J. Am. Chem. Soc. 2001, 123, 75397552.

[16] L. Poorters, D. Armspach, D. Matt, L. Toupet, P. G. Jones, Angew. Chem. Int. Ed. Engl. 2007, 46, 2663-2665.

[17] P. D. Beer, V. Timoshenko, M. Maestri, P. Passaniti, V. Balzani, Chem. Comm. 1999, 1755-1756.

[18] A. J. Evans, S. E. Matthews, A. R. Cowley, P. D. Beer, Dalton 2003, 4644-4650.

[19] S. Leininger, B. Olenyuk, P. J. Stang, Chem. Rev. 2000, 100, 853-908. 
[20] Y. Yamanoi, Y. Sakamoto, T. Kusukawa, M. Fujita, S. Sakamoto, K. Yamaguchi, J. Am. Chem. Soc. 2001, 123, 980-981.

[21] D. Zuccaccia, L. Pirondini, R. Pinalli, E. Dalcanale, A. Macchioni, J. Am. Chem. Soc. 2005, 127, 7025-7032.

[22] V. Maurizot, M. Yoshizawa, M. Kawano, M. Fujita, Dalton 2006, 2750-2756.

[23] R. J. Puddephatt, Can. J. Chem. 2006, 84, 1505-1514.

[24] M. D. Pluth, R. G. Bergman, K. Raymond, in Supramolecular Catalysis (Ed. P. W. N. M. van Leeuwen), Wiley-VCH, Weinheim, 2008, pp 165-197.

[25] S. Sameni, C. Jeunesse, D. Matt, L. Toupet, Chem. Eur. J. 2009, 15, 10446-10456.

[26] C. B. Dieleman, D. Matt, I. Neda, R. Schmutzler, H. Thönnessen, P. G. Jones, A. Harriman, Dalton 1998, 2115-2121.

[27] D. Armspach, L. Poorters, D. Matt, B. Benmerad, F. Balegroune, L. Toupet, Org. Biomol. Chem. 2005, 3, 2588-2592.

[28] M. R. Eberhard, K. M. Heslop, A. G. Orpen, P. G. Pringle, Organometallics 2005, 24, 335-337.

[29] O. Grossman, C. Azerraf, D. Gelman, Organometallics 2006, 25, 375-381.

[30] P. Braunstein, D. Matt, F. Mathey, D. Thavard, J. Chem. Res. (S) 1978, 232-233.

[31] J. C. Jeffrey, T. B. Rauchfuss, Inorg. Chem. 1979, 18, 2658-2666.

[32] A. Bader, E. Lindner, Coord. Chem. Rev. 1991, 108, 27-110.

[33] T. Hara, T. Yamagata, K. Mashima, Organometallics 2007, 26, 110-118.

[34] G. Parkin, Acc. Chem. Res. 1992, 25, 455-460.

[35] P. Comba, A. hauser, M. Kerscher, H. Pritzkow, Angew. Chem. Int. Ed. Engl. 2003, $42,4356-4540$.

[36] As expected, the isomeric phosphine-phosphine oxide, $i$. e. the one in which the phosphoryl group bridges the A and B glucose units, also forms. This ligand was isolated as a chelate complex (see SI).

[37] L. Poorters, D. Armspach, D. Matt, L. Toupet, S. Choua, P. Turek, Chem. Eur. J. 2007, 13, 9448-9461. 


\section{Graphical Abstract}

\section{A cavity-shaped diphosphane displaying os-chelating behaviour}

Rafael Gramage-Doria, Dominique Armspach, Dominique Matt, Loic Toupet

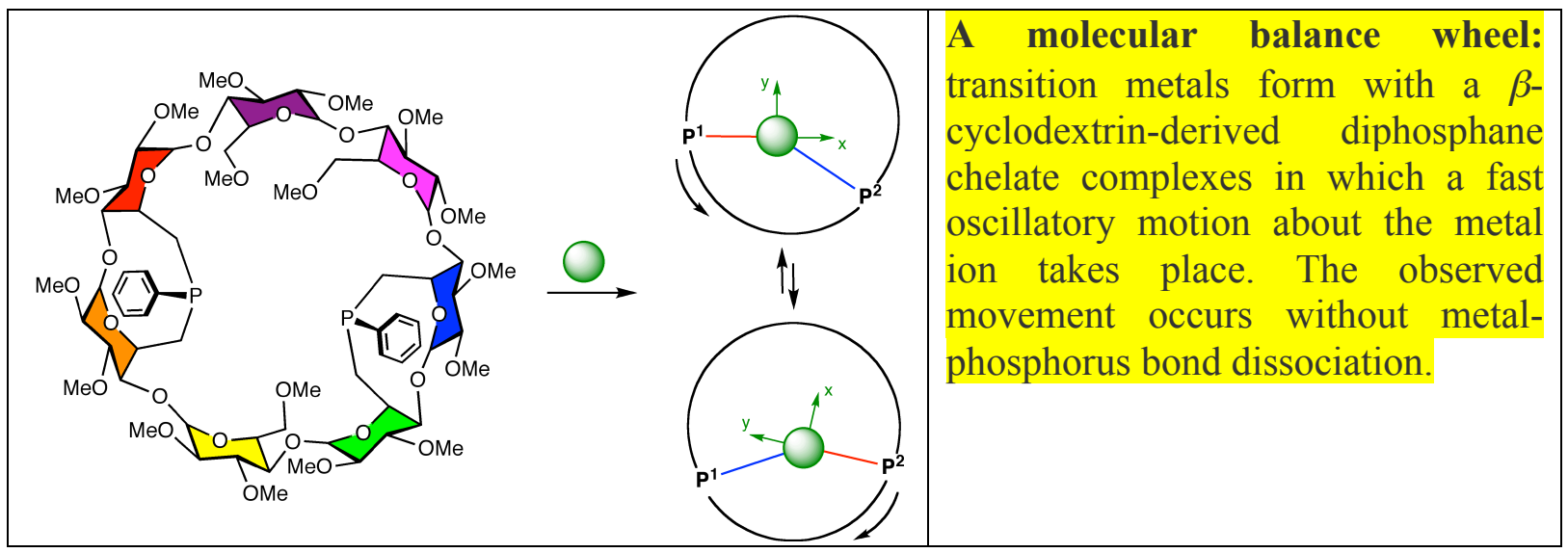




\title{
Supporting Information
}

\author{
Angewandte Chemie
}

\section{A cavity-shaped diphosphane displaying os-chelating behaviour}

Rafael Gramage-Doria, Dominique Armspach, Dominique Matt, and Loic Toupet

\section{Content}

- General methods

- Synthesis and characterisation of 2-13

- Crystal structure analyses of $\mathbf{7} \bullet 0.5 \mathrm{CH}_{2} \mathrm{Cl}_{2} \bullet 0.5 \mathrm{C}_{5} \mathrm{H}_{12}, \mathbf{1 2} \cdot 0.5 \mathrm{C}_{5} \mathrm{H}_{12}$, and $13 \cdot \mathrm{C}_{5} \mathrm{H}_{12}$.

- Figure SI-1. ${ }^{31} \mathrm{P}\left\{{ }^{1} \mathrm{H}\right\}$ NMR spectrum and ${ }^{31} \mathrm{P}\left\{{ }^{1} \mathrm{H}\right\}-{ }^{31} \mathrm{P}\left\{{ }^{1} \mathrm{H}\right\}$ COSY $2 \mathrm{D}$ NMR spectrum of complex 10 at $-80^{\circ} \mathrm{C}$ recorded in $\mathrm{CD}_{2} \mathrm{Cl}_{2}$ at $202.5 \mathrm{MHz}$

- Figure SI-2. ${ }^{31} \mathrm{P}\left\{{ }^{1} \mathrm{H}\right\}$ NMR spectrum and off resonance ${ }^{31} \mathrm{P}\left\{{ }^{1} \mathrm{H}\right\}-{ }^{31} \mathrm{P}\left\{{ }^{1} \mathrm{H}\right\}$ ROESY NMR spectrum of complex 10 at $-60^{\circ} \mathrm{C}$ recorded in $\mathrm{CD}_{2} \mathrm{Cl}_{2}$ at $202.5 \mathrm{MHz}$.

- Figure SI-3. ${ }^{1} \mathrm{H}$ NMR spectrum recorded in $\mathrm{CDCl}_{3}$ at $25^{\circ} \mathrm{C}$ at $300.1 \mathrm{MHz}$ (left) and ${ }^{1} \mathrm{H}$ NMR spectra showing the anomeric protons zone in the range $-80 / 25^{\circ} \mathrm{C}$ recorded in $\mathrm{CD}_{2} \mathrm{Cl}_{2}$ at $500.1 \mathrm{MHz}$ (left) of complex $\mathbf{1 0}$.

- Figure SI-4. ${ }^{31} \mathrm{P}\left\{{ }^{1} \mathrm{H}\right\}$ NMR spectra in the range $25^{\circ} \mathrm{C} / 70^{\circ} \mathrm{C}$ at $121.5 \mathrm{MHz}$ (left) and ${ }^{31} \mathrm{P}\left\{{ }^{1} \mathrm{H}\right\} \mathrm{NMR}$ spectrum at $80^{\circ} \mathrm{C}$ at $202.5 \mathrm{MHz}$ (right) recorded in $\mathrm{C}_{2} \mathrm{D}_{2} \mathrm{Cl}_{4}$ of complex 10.

\section{General Methods}

All commercial reagents were used as supplied. Solvents were dried by conventional methods and distilled immediately prior to use. $\mathrm{CDCl}_{3}$ was passed down a $5 \mathrm{~cm}$-thick alumina column and stored under nitrogen over molecular sieves $(4 \AA)$. Routine ${ }^{1} \mathrm{H}$, and ${ }^{13} \mathrm{C}\left\{{ }^{1} \mathrm{H}\right\}$ NMR spectra were recorded on FT Bruker AVANCE 300, AVANCE 400, AVANCE 500 and AVANCE 600 instruments. ${ }^{1} \mathrm{H}$ NMR spectral data were referenced to residual protiated solvents $[7.26$ ppm for $\mathrm{CDCl}_{3}, 7.16$ ppm for $\mathrm{C}_{6} \mathrm{D}_{6}$ and 5.32 for $\left.\mathrm{CD}_{2} \mathrm{Cl}_{2}\right],{ }^{13} \mathrm{C}$ chemical shifts are reported relative to deuterated solvents $\left[77.0 \mathrm{ppm}\right.$ for $\mathrm{CDCl}_{3}, 128.06 \mathrm{ppm}$ for $\mathrm{C}_{6} \mathrm{D}_{6}$ and for 54.00 $\mathrm{CD}_{2} \mathrm{Cl}_{2}$ ] and the ${ }^{31} \mathrm{P}$ NMR data are given relative to external $\mathrm{H}_{3} \mathrm{PO}_{4}$. Mass spectra were 
recorded either on a Maldi TOF spectrometer (MALDI-TOF) using $\alpha$-cyano-4hydroxycinnamic acid as matrix, or on a Bruker MicroTOF spectrometer (ESI-TOF) using $\mathrm{CH}_{2} \mathrm{Cl}_{2}, \mathrm{MeCN}$ or $\mathrm{MeOH}$ as the solvent. Elemental analyses were performed by the Service de Microanalyse, Institut de Chimie (UMR 7177 CNRS), Strasbourg. Melting points were determined with a Büchi 535 capillary melting-point apparatus. The dialkylating reagent $\mathbf{1}{ }^{1}$ $\mathrm{PtCl}_{2}(\mathrm{PhCN})_{2},{ }^{2}$ and $\mathrm{PdCl}_{2}(\mathrm{PhCN})_{2}{ }^{2}$ were prepared according to described procedures. Assignment of the stereochemistry of the $\mathrm{P}$ atoms was made by giving arbitrarily priority to glucose units $A$ and $D$ over glucose units $B$ and $E$, respectively. The numbering of the atoms within a glucose unit is as shown below.
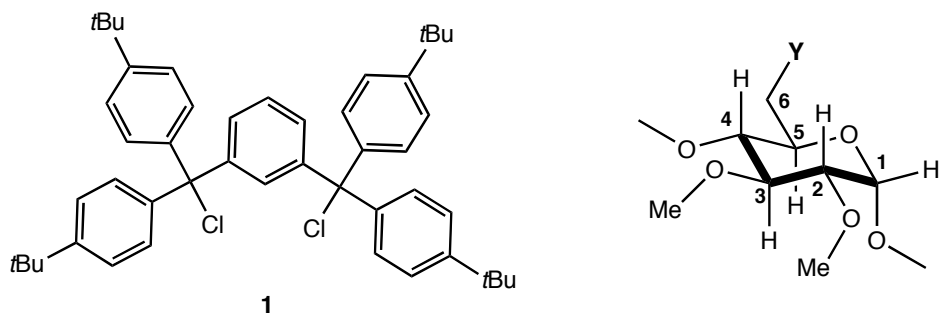

$6^{\mathrm{A}}, 6^{\mathrm{B}}, 6^{\mathrm{D}}, 6^{\mathrm{E}}$-Tetra-O-6 $6^{\mathrm{A}}, 6^{\mathrm{B}}: 6^{\mathrm{D}}, 6^{\mathrm{E}}$-bis $\{$ benzene-1,3-bis[bis(4-tert-butylphenyl)methyl]\}$2^{\mathrm{A}}, 2^{\mathrm{B}}, 2^{\mathrm{C}}, 2^{\mathrm{D}}, 2^{\mathrm{E}}, 2^{\mathrm{F}}, 2^{\mathrm{G}}, 3^{\mathrm{A}}, 3^{\mathrm{B}}, 3^{\mathrm{C}}, 3^{\mathrm{D}}, 3^{\mathrm{E}}, 3^{\mathrm{F}}, 3^{\mathrm{G}}, 6^{\mathrm{C}}, 6^{\mathrm{F}}, 6^{\mathrm{G}}$-heptadeca- $O$-methyl- $\beta$-cyclodextrin (2)

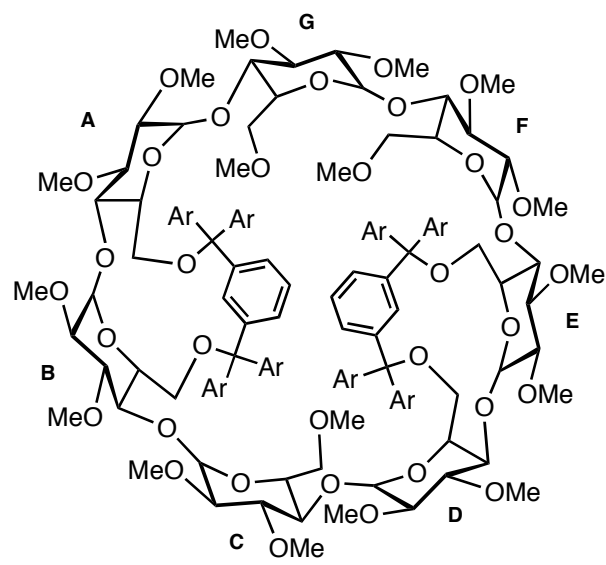

1,3-bis[bis(4-tert-butylphenyl)chloromethyl]benzene 1 (5.42 $\mathrm{g}, 7.7 \mathrm{mmol})$ was added to a solution of $\beta$-cyclodextrin $(3.97 \mathrm{~g}, 3.5 \mathrm{mmol})$ and DMAP $(0.51 \mathrm{~g}, 4.2 \mathrm{mmol})$ in pyridine (90 $\mathrm{mL}$ ). The reaction mixture was stirred at $70^{\circ} \mathrm{C}$ for $12 \mathrm{~h}$ before being cooled down to room temperature. Pyridine was then removed in vacuo. Addition of water $(500 \mathrm{~mL})$ to the residue produced a suspension that was filtrated. The cake was dried in vacuo at $50^{\circ} \mathrm{C}$ for $12 \mathrm{~h}$. The 
colourless solid was dissolved in DMF $(150 \mathrm{~mL})$ and $\mathrm{NaH}(5.88 \mathrm{~g}, 147 \mathrm{mmol})$ was added carefully followed by the addition of catalytic amounts of imidazole $(0.010 \mathrm{~g}, 0.15 \mathrm{mmol})$. The reaction mixture was stirred at room temperature for $1 \mathrm{~h}$ before being cooled at $0^{\circ} \mathrm{C}$ whereupon MeI (17.88 g, $7.8 \mathrm{~mL}, 126 \mathrm{mmol}$ ) was added dropwise at $0^{\circ} \mathrm{C}$. The yellow suspension was stirred for $12 \mathrm{~h}$ at room temperature. $\mathrm{MeOH}(50 \mathrm{~mL})$ was then added slowly to quench excess $\mathrm{NaH}$. The reaction mixture was poured into water $(500 \mathrm{~mL})$ under stirring before being extracted with $\mathrm{Et}_{2} \mathrm{O}(3 \times 300 \mathrm{~mL})$. The organic exctract was dried $\left(\mathrm{MgSO}_{4}\right)$ and evaporated to dryness to afford a brown residue. The crude material was purified by column chromatography $\left(\mathrm{SiO}_{2}\right.$, petroleum ether/AcOEt, 80:20 to $\left.65: 35, v / v\right)$ to give the desired product $2(4.63 \mathrm{~g}, 50 \%)$ as a colourless solid. $R_{\mathrm{f}}\left(\mathrm{SiO}_{2}\right.$, petroleum ether/AcOEt, 60:40, v/v) $=$ 0.60; m.p. dec.; ${ }^{1} \mathrm{H}$ NMR $\left(300.1 \mathrm{MHz}, \mathrm{CDCl}_{3}, 25^{\circ} \mathrm{C}\right): \delta$ (assignment by COSY) $=1.17$ (s, 6 $\mathrm{H}, t \mathrm{Bu}), 1.18(\mathrm{~s}, 6 \mathrm{H}, t \mathrm{Bu}), 1.20(\mathrm{~s}, 6 \mathrm{H}, t \mathrm{Bu}), 1.24(\mathrm{~s}, 6 \mathrm{H}, t \mathrm{Bu}), 1.27-1.29(48 \mathrm{H}, t \mathrm{Bu}), 2.69$ $\left(\mathrm{d}, 1 \mathrm{H},{ }^{3} J_{\mathrm{H}-2, \mathrm{H}-1}=4.0 \mathrm{~Hz}, \mathrm{H}-2\right), 2.72(\mathrm{~s}, 3 \mathrm{H}, \mathrm{OMe}), 2.75\left(\mathrm{~d}, 1 \mathrm{H},{ }^{3} J_{\mathrm{H}-2, \mathrm{H}-1}=3.7 \mathrm{~Hz}, \mathrm{H}-2\right)$, 3.06 (s, $3 \mathrm{H}, \mathrm{OMe}), 3.26$ (s, $3 \mathrm{H}, \mathrm{OMe}), 3.33$ (s, $3 \mathrm{H}, \mathrm{OMe}), 3.36$ (s, $3 \mathrm{H}, \mathrm{OMe}), 3.52$ (s, $3 \mathrm{H}$, OMe), 3.56 (s, 3 H, OMe), 3.59 (s, 3 H, OMe), 3.61 (s, 3 H, OMe), 3.62 (s, 3 H, OMe), 3.63 (s, $9 \mathrm{H}, \mathrm{OMe}), 3.64$ (s, $3 \mathrm{H}, \mathrm{OMe}), 3.65$ (s, $3 \mathrm{H}, \mathrm{OMe}), 3.66(\mathrm{~s}, 3 \mathrm{H}, \mathrm{OMe}), 3.67$ (s, $3 \mathrm{H}$, OMe), 3.20-4.05 (38 H, H-2, H-3, H-4, H-5, H-6a $\left.{ }^{\text {A,D }}, \mathrm{H}-6^{\mathrm{B}, \mathrm{C}, \mathrm{E}, \mathrm{F}, \mathrm{G}}\right), 4.22-4.28(3 \mathrm{H}, \mathrm{H}-1, \mathrm{H}-$ $\left.6 b^{\mathrm{A}, \mathrm{D}}\right), 4.50\left(\mathrm{~d}, 1 \mathrm{H},{ }^{3} J_{\mathrm{H}-1, \mathrm{H}-2}=3.5 \mathrm{~Hz}, \mathrm{H}-1\right), 5.15\left(\mathrm{~d}, 1 \mathrm{H},{ }^{3} J_{\mathrm{H}-1, \mathrm{H}-2}=3.4 \mathrm{~Hz}, \mathrm{H}-1\right), 5.37-5.39$ (2 H, H-1), 5.49-5.51 (2 H, H-1), 6.99 (t, $2 \mathrm{H},{ }^{3} J_{\mathrm{H} 5-\mathrm{H} 4}=6.5 \mathrm{~Hz}, \mathrm{H}-5$ of both bridging $\left.\mathrm{C}_{6} \mathrm{H}_{4}\right)$, $7.05-7.53(36 \mathrm{H}$, aromatic $\mathrm{H}), 7.62\left(\mathrm{~s}, 1 \mathrm{H}, \mathrm{H}-2\right.$ of bridging $\left.\mathrm{C}_{6} \mathrm{H}_{4}\right), 7.73(\mathrm{~s}, 1 \mathrm{H}, \mathrm{H}-2$ of bridging $\left.\mathrm{C}_{6} \mathrm{H}_{4}\right)$ ppm; ${ }^{13} \mathrm{C}\left\{{ }^{1} \mathrm{H}\right\}$ NMR $\left(75.5 \mathrm{MHz} \mathrm{CDCl}_{3}, 25^{\circ} \mathrm{C}\right): \delta$ (assignment by HMQC) $=$ $31.43[\times 24](\mathrm{Me}$ of $t \mathrm{Bu}), 34.3$ [×8] (C of $t \mathrm{Bu}), 57.80,57.88,58.06,58.14,58.25,58.42,58.80$, 59.03, 59.51, 59.70, 60.60, 61.03, 61.52, 61.70, 61.75, 61.84, 61.98 (OMe), 61.68, 61.70, 62.73, $63.18\left(\mathrm{C}-6^{\mathrm{A}, \mathrm{B}, \mathrm{D}, \mathrm{E})}, 71.09,71.75,72.54\left(\mathrm{C}-6^{\mathrm{C}, \mathrm{F}, \mathrm{G}}\right), 70.46,70.49,70.61,70.76,71.20\right.$, 71.57, 71.65 (C-5), 77.21, 78.74, 80.19, 80.54, 80.98, 81.04, 81.19, 81.27, 81.64, 81.77 [×4], $82.13,82.23,82.26,82.59$ [×2], 82.74 [×2], 82.86 (C-2, C-3, C-4), 85.74, 86.11, 87.51, 87.85 [OC $\left.(\mathrm{Ar})_{3}\right], 97.74,98.24,98.51,98.63,98.77$ [×3] (C-1), 124.11 [×5], 124.33, 124.43, 124.48 [×2], 124.59, 126.29, 126.44, 126.50, 126.83, 127.28, 127.46, 128.11, 128.14, 130.52, 131.00, 131.53, 131.88, 134.37, 134.95 ( $o-\mathrm{C}$ and $m-\mathrm{C}), 140.09,140.20,140.41,140.59,140.94$, $141.59,141.92,142.37,142.83,144.58,144.96,145.24,148.46,148.52,148.54,148.56$, $148.60,148.66[\times 2], 148.92$ (ipso-C) ppm; elemental analysis (\%) calcd for $\mathrm{C}_{155} \mathrm{H}_{212} \mathrm{O}_{35} \cdot \mathrm{CH}_{2} \mathrm{Cl}_{2}(2635.32+83.95): \mathrm{C}$ 68.88, $\mathrm{H}$ 7.93, found: $\mathrm{C}$ 68.77, $\mathrm{H}$ 8.09; MS (ESITOF): $m / z$ (\%): $2657.38(100)[M+\mathrm{Na}]^{+}$. 


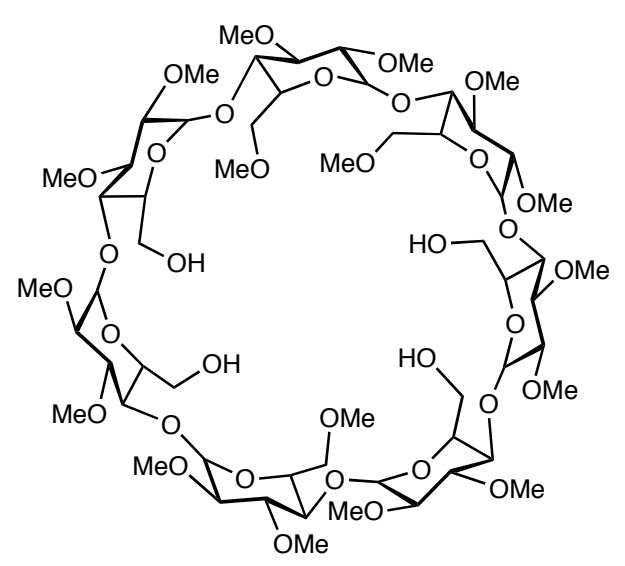

$\mathrm{HBF}_{4}(34 \mathrm{wt} \%$ aq, $7.5 \mathrm{~g}, 7.5 \mathrm{~mL}, 29 \mathrm{mmol}$ ) was added dropwise to a stirred solution of capped cyclodextrin $2(2.55 \mathrm{~g}, 0.97 \mathrm{mmol})$ in $\mathrm{MeCN}(30 \mathrm{~mL})$ at room temperature. After 30 min, $\mathrm{NEt}_{3}(1.47 \mathrm{~g}, 2.0 \mathrm{~mL})$ was added dropwise under stirring. Addition of water $(100 \mathrm{~mL})$ to the reaction mixture caused the carbinol to precipitate. The resulting suspension was filtrated and the filtrate extracted with $\mathrm{CHCl}_{3}(3 \times 50 \mathrm{~mL})$. The combined organic extracts were washed with a saturated aqueous $\mathrm{NaHCO}_{3}$ solution $(100 \mathrm{~mL})$ before being dried $\left(\mathrm{MgSO}_{4}\right)$. Removal of the solvent in vacuo gave tetrol $3(1.32 \mathrm{~g}, 99 \%)$ as a colourless solid. $R_{\mathrm{f}}\left(\mathrm{SiO}_{2}\right.$, $\left.\mathrm{CH}_{2} \mathrm{Cl}_{2} / \mathrm{MeOH}, 92: 8, v / v\right)=0.18$; m.p. $153^{\circ} \mathrm{C} ;{ }^{1} \mathrm{H}$ NMR $\left(300.1 \mathrm{MHz}, \mathrm{CDCl}_{3}, 25^{\circ} \mathrm{C}\right): \delta$ (assignment by COSY) $=2.61\left(\mathrm{t}, 1 \mathrm{H},{ }^{3} J_{\mathrm{OH}-\mathrm{H}-6}=6.2 \mathrm{~Hz}, \mathrm{OH}\right), 2.68\left(\mathrm{t}, 1 \mathrm{H},{ }^{3} J_{\mathrm{OH}-\mathrm{H}-6}=5.9 \mathrm{~Hz}\right.$, $\mathrm{OH}), 2.78\left(\mathrm{t}, 1 \mathrm{H},{ }^{3} J_{\mathrm{OH}-\mathrm{H}-6}=6.1 \mathrm{~Hz}, \mathrm{OH}\right), 2.96\left(\mathrm{t}, 1 \mathrm{H},{ }^{3} J_{\mathrm{OH}-\mathrm{H}-6}=5.8 \mathrm{~Hz}, \mathrm{OH}\right), 3.17-3.22(7 \mathrm{H}$, H-2), 3.30-4.00 (35 H, H-3, H-4, H-5, H-6) 3.37 (s, 9 H, OMe-6), 3.49 (s, 9 H, OMe), 3.51 (s, $6 \mathrm{H}, \mathrm{OMe}$ ), 3.52 (s, $3 \mathrm{H}, \mathrm{OMe}$ ), 3.53 (s, $3 \mathrm{H}, \mathrm{OMe}$ ), 3.62 (s, $9 \mathrm{H}, \mathrm{OMe}), 3.63$ (s, $3 \mathrm{H}, \mathrm{OMe}$ ), $3.64(\mathrm{~s}, 6 \mathrm{H}, \mathrm{OMe}), 3.65(\mathrm{~s}, 3 \mathrm{H}, \mathrm{OMe}), 5.01-5.03(2 \mathrm{H}, \mathrm{H}-1), 5.09\left(\mathrm{~d}, 1 \mathrm{H},{ }^{2} J_{\mathrm{H}-1, \mathrm{H}-2}=3.8 \mathrm{~Hz}\right.$, $\mathrm{H}-1), 5.10\left(\mathrm{~d}, 1 \mathrm{H},{ }^{2} J_{\mathrm{H}-1, \mathrm{H}-2}=3.8 \mathrm{~Hz}, \mathrm{H}-1\right), 5.17$ (d, $\left.2 \mathrm{H},{ }^{2} J_{\mathrm{H}-1, \mathrm{H}-2}=3.5 \mathrm{~Hz}, \mathrm{H}-1\right), 5.23(\mathrm{~d}, 1 \mathrm{H}$, $\left.{ }^{2} J_{\mathrm{H}-1, \mathrm{H}-2}=3.8 \mathrm{~Hz}, \mathrm{H}-1\right) \mathrm{ppm} ;{ }^{13} \mathrm{C}\left\{{ }^{1} \mathrm{H}\right\} \mathrm{NMR}\left(75.5 \mathrm{MHz} \mathrm{CDCl}_{3}, 25{ }^{\circ} \mathrm{C}\right.$ ): $\delta$ (assignment by $\mathrm{HMQC})=58.25[\times 3], 58.68,58.77,58.95,59.00,59.06,59.19[\times 2], 61.05,61.09,61.25$, $61.28,61.53,61.63,61.78(\mathrm{OMe}), 61.78,61.97[\times 2], 62.05\left(\mathrm{C}-6^{\mathrm{A}, \mathrm{B}, \mathrm{D}, \mathrm{E}}\right), 71.18,71.32,71.47$ $\left(\mathrm{C}-5^{\mathrm{C}, \mathrm{F}, \mathrm{G}}\right), 71.39,71.54,71.69\left(\mathrm{C}-6^{\mathrm{C}, \mathrm{F}, \mathrm{G}}\right), 71.83,72.03,72.33,72.53\left(\mathrm{C}-5^{\mathrm{A}, \mathrm{B}, \mathrm{D}, \mathrm{E}}\right), 78.29,79.11$ [×2] 79.96, 80.44, 80.69, 81.09, 81.24, 81.41,81.45,81.60 [×2], 81.73,81.77 [×2], 81.98 [×4], 82.07, 82.14 (C-2, C-3, C-4), 98.37, 98.60, 98.70 [×2], 98.85 [×2], 98.92 (C-1) ppm; elemental analysis (\%) calcd for $\mathrm{C}_{59} \mathrm{H}_{104} \mathrm{O}_{35}$ (1373.44): C 51.60 , H 7.63 , found: $\mathrm{C} 51.68, \mathrm{H}$ 7.57; MS (ESI-TOF): $m / z(\%): 1395.63(100)[M+\mathrm{Na}]^{+}$. 
$6^{\mathrm{A}}, \mathbf{6}^{\mathrm{B}}, \mathbf{6}^{\mathrm{D}}, \mathbf{6}^{\mathrm{E}}-\boldsymbol{O}$-Tetramethylsulfonyl-2 $2^{\mathrm{A}}, 2^{\mathrm{B}}, 2^{\mathrm{C}}, 2^{\mathrm{D}}, 2^{\mathrm{E}}, 2^{\mathrm{F}}, 2^{\mathrm{G}}, 3^{\mathrm{A}}, 3^{\mathrm{B}}, 3^{\mathrm{C}}, 3^{\mathrm{D}}, \mathbf{3}^{\mathrm{E}}, 3^{\mathrm{F}}, 3^{\mathrm{G}}, \mathbf{6}^{\mathrm{C}}, \mathbf{6}^{\mathrm{F}}, \mathbf{6}^{\mathrm{G}}-$ heptadeca- $O$-methyl- $\beta$-cyclodextrin (4)

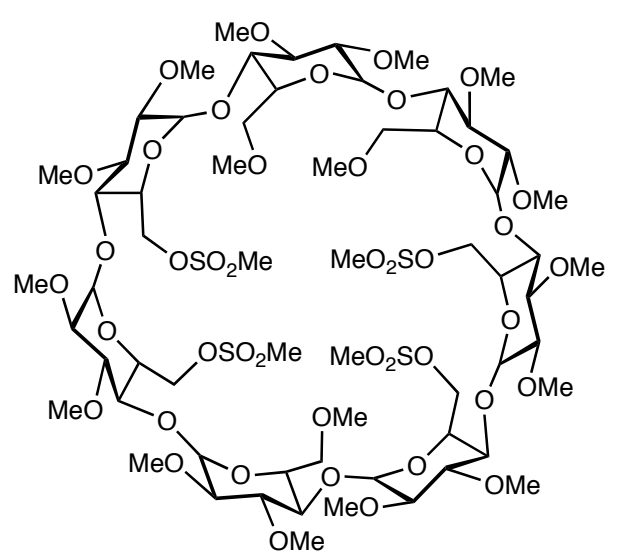

Methanesulfonyl chloride $(0.34 \mathrm{~g}, 0.23 \mathrm{~mL}, 2.94 \mathrm{mmol})$ was added to a solution of tetrol 3 $(0.96 \mathrm{~g}, 0.70 \mathrm{mmol})$ and DMAP $(0.35 \mathrm{~g}, 2.87 \mathrm{mmol})$ in dry pyridine $(30 \mathrm{~mL})$ at $0^{\circ} \mathrm{C}$. The reaction mixture was stirred at room temperature for $12 \mathrm{~h}$ before adding water $(100 \mathrm{~mL})$. The solution was extracted with AcOEt $(3 \times 50 \mathrm{~mL})$. The combined organic extracts were washed sequentially with $\mathrm{HCl} 2 \mathrm{M}(2 \times 50 \mathrm{~mL}), \mathrm{NaOH} 2 \mathrm{M}(2 \times 50 \mathrm{~mL})$ and water $(50 \mathrm{~mL})$ before being dried $\left(\mathrm{MgSO}_{4}\right)$. Removal of the solvent in vacuo gave pure tetramesylate $4(1.14 \mathrm{~g}$, $97 \%)$ as a colourless solid. $R_{\mathrm{f}}\left(\mathrm{SiO}_{2}, \mathrm{CH}_{2} \mathrm{Cl}_{2} / \mathrm{MeOH}, 92: 8, v / v\right)=0.40$; m.p. $201^{\circ} \mathrm{C} ;{ }^{1} \mathrm{H} \mathrm{NMR}$ $\left(300.1 \mathrm{MHz}, \mathrm{CDCl}_{3}, 25^{\circ} \mathrm{C}\right): \delta$ (assignment by $\left.\mathrm{COSY}\right)=3.06\left(\mathrm{~s}, 6 \mathrm{H}, \mathrm{OSO}_{2} \mathrm{Me}\right), 3.07(\mathrm{~s}, 3 \mathrm{H}$, $\mathrm{OSO}_{2} \mathrm{Me}$ ), 3.07 (s, $3 \mathrm{H}, \mathrm{OSO}_{2} \mathrm{Me}$ ), 3.14-3.21 (7 H, H-2), 3.37 (s, $\left.3 \mathrm{H}, \mathrm{OMe}\right), 3.38$ (s, $3 \mathrm{H}$, OMe), 3.39 (s, 3 H, OMe), 3.49 (s, 9 H, OMe), 3.50 (s, 6 H, OMe), 3.53 (s, $6 \mathrm{H}, \mathrm{OMe}$ ), 3.60 (s, $3 \mathrm{H}, \mathrm{OMe}), 3.61$ (s, $3 \mathrm{H}, \mathrm{OMe}), 3.62$ (s, $6 \mathrm{H}, \mathrm{OMe}), 3.64$ (s, $3 \mathrm{H}, \mathrm{OMe}), 3.66$ (s, $3 \mathrm{H}$, OMe), 3.68 (s, 3 H, OMe), 3.40-4.05 (27 H, H-3, H-4, H-5, H-6 $\left.{ }^{\mathrm{C}, \mathrm{F}, \mathrm{G}}\right), 4.32-4.34$ (2 H, H$\left.6 \mathrm{a}^{\mathrm{A}, \mathrm{D} \text { or B,E }}\right), 4.53-4.70\left(6 \mathrm{H}, \mathrm{H}-6 \mathrm{a}^{\mathrm{B}, \mathrm{E} \text { or A,D }}, \mathrm{H}-6 \mathrm{~b}^{\mathrm{A}, \mathrm{B}, \mathrm{D}, \mathrm{E}}\right), 5.08-5.11(4 \mathrm{H}, \mathrm{H}-1), 5.14\left(\mathrm{~d}, 1 \mathrm{H},{ }^{3} J_{\mathrm{H}-}\right.$ $1, \mathrm{H}-2=3.6 \mathrm{~Hz}, \mathrm{H}-1), 5.20\left(\mathrm{~d}, 1 \mathrm{H},{ }^{3} J_{\mathrm{H}-1, \mathrm{H}-2}=3.9 \mathrm{~Hz}, \mathrm{H}-1\right), 5.22\left(\mathrm{~d}, 1 \mathrm{H},{ }^{3} J_{\mathrm{H}-1, \mathrm{H}-2}=3.9 \mathrm{~Hz}, \mathrm{H}-1\right)$ ppm; ${ }^{13} \mathrm{C}\left\{{ }^{1} \mathrm{H}\right\}$ NMR $\left(75.5 \mathrm{MHz} \mathrm{CDCl}_{3}, 25^{\circ} \mathrm{C}\right): \delta$ (assignment by $\left.\mathrm{HMQC}\right)=37.17,37.30$ $[\times 2], 37.50\left(\mathrm{OSO}_{2} \mathrm{Me}\right), 58.26,58.39[\times 2], 58.46,58.74,59.10,59.15[\times 2], 59.36,59.44$, $61.11,61.21,61.62[\times 3], 61.76,61.80(\mathrm{OMe}), 69.32[\times 2], 69.88[\times 2]\left(\mathrm{C}-6^{\mathrm{A}, \mathrm{B}, \mathrm{D}, \mathrm{E}}\right), 69.56[\times 2]$, $69.67[\times 2]\left(\mathrm{C}-5^{\mathrm{A}, \mathrm{B}, \mathrm{D}, \mathrm{E}}\right), 70.87,70.96[\times 2]\left(\mathrm{C}-6^{\mathrm{C}, \mathrm{F}, \mathrm{G}}\right), 71.16[\times 2], 71.32\left(\mathrm{C}-5^{\mathrm{C}, \mathrm{F}, \mathrm{G}}\right), 78.10,78.35$, $80.13,80.20,80.63,80.67$ [×2], 81.01, 81.47, 81.61 [×5], 81.69, 81.75, 81.85 [×3], 81.95, 82.04 (C-2, C-3, C-4), 97.66, 98.41, 98.94 [×2], 99.12 [×2], 99.17 (C-1) ppm; elemental 
analysis (\%) calcd for $\mathrm{C}_{63} \mathrm{H}_{112} \mathrm{O}_{43} \mathrm{~S}_{4}$ (1685.80): C 44.89, $\mathrm{H}$ 6.70, found: $\mathrm{C} 44.88$, H 6.65; MS (ESI-TOF): $m / z(\%): 1707.54(100)[M+\mathrm{Na}]^{+}$.

$P, P^{\prime}-\left\{6^{\mathrm{A}}, 6^{\mathrm{B}}, 6^{\mathrm{D}}, 6^{\mathrm{E}}-\right.$ Tetradeoxy- $6^{\mathrm{A}}, 6^{\mathrm{B}}: 6^{\mathrm{D}}, 6^{\mathrm{E}}-$ bis $[(R)$-phenylphosphinidene $]-2^{\mathrm{A}}, 2^{\mathrm{B}}, 2^{\mathrm{C}}, 2^{\mathrm{D}}, 2^{\mathrm{E}}$, $2^{\mathrm{F}}, 2^{\mathrm{G}}, 3^{\mathrm{A}}, 3^{\mathrm{B}}, 3^{\mathrm{C}}, 3^{\mathrm{D}}, 3^{\mathrm{E}}, 3^{\mathrm{F}}, 3^{\mathrm{G}}, 6^{\mathrm{C}}, 6^{\mathrm{F}}, 6^{\mathrm{G}}$-heptadeca- $O$-methyl- $\beta$-cyclodextrin $\}$ diborane (5)

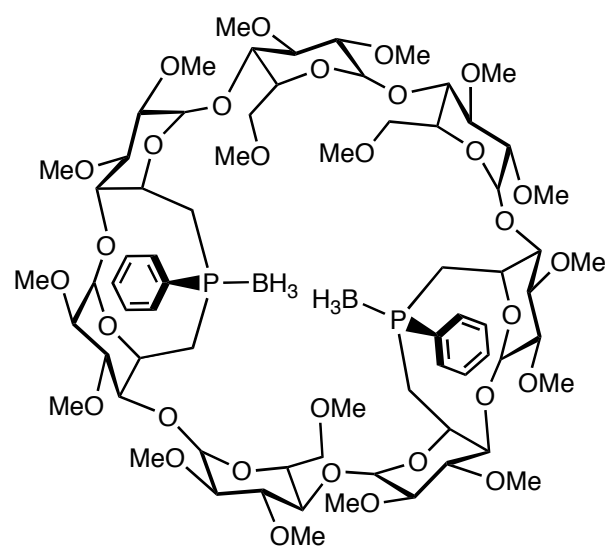

A solution of ${ }^{n} \mathrm{BuLi}$ in hexane $(1.60 \mathrm{M}, 1.22 \mathrm{~mL}, 1.96 \mathrm{mmol})$ was added dropwise to a stirred solution of $\mathrm{H}_{2} \mathrm{PPh}(0.098 \mathrm{~g}, 0.89 \mathrm{mmol}, 0.098 \mathrm{~mL})$ in THF $(16.5 \mathrm{~mL})$ at $-78{ }^{\circ} \mathrm{C}$. The yellow solution was allowed to rise to room temperature over $1 \mathrm{~h}$ whereupon the phosphide dianion precipitated. The resulting yellow suspension was cannulated slowly, within $1 \mathrm{~h}$, into a stirred solution of tetramesylate $4(0.300 \mathrm{~g}, 0.18 \mathrm{mmol})$ in THF $(25 \mathrm{~mL})$. The reaction mixture was stirred for $12 \mathrm{~h}$ at room temperature. The solvent was then removed in vacuo and excess of $\mathrm{Li}_{2} \mathrm{PPh}$ was protonated with $\mathrm{MeOH}(15 \mathrm{~mL})$. After removal of the solvent in vacuo, toluene $(100 \mathrm{~mL})$ was added and the resulting suspension filtered over celite. Evaporation of the solvent gave a colourless residue which was dissolved in THF $(10 \mathrm{~mL})$ before adding a solution of $\mathrm{BH}_{3} \cdot \mathrm{THF}$ in THF $(1.00 \mathrm{M}, 0.9 \mathrm{~mL}, 0.9 \mathrm{mmol})$ dropwise at $0^{\circ} \mathrm{C}$. After stirring for $12 \mathrm{~h}$ at room temperature, the solvent was removed in vacuo and the resulting colourless residue subjected to column chromatography (dried $\mathrm{SiO}_{2}, \mathrm{CH}_{2} \mathrm{Cl}_{2} / \mathrm{MeOH}, 97: 3, v / v$ ) to afford pure $5(0.120 \mathrm{~g}, 40 \%)$ as a colourless solid. $R_{\mathrm{f}}\left(\mathrm{SiO}_{2}, \mathrm{CH}_{2} \mathrm{Cl}_{2} / \mathrm{MeOH}, 92: 8, v / v\right)=0.35$; m.p. $185^{\circ} \mathrm{C} ;{ }^{1} \mathrm{H}$ NMR $\left(300.1 \mathrm{MHz}, \mathrm{CDCl}_{3}, 25^{\circ} \mathrm{C}\right): \delta$ (assignment by COSY) $=0.89(\mathrm{br} \mathrm{s}, 6 \mathrm{H}, \mathrm{P}-$ $\left.\mathrm{BH}_{3}\right), 1.62-1.64\left(4 \mathrm{H}, \mathrm{H}-6 \mathrm{a}^{\mathrm{A}, \mathrm{B}, \mathrm{D}, \mathrm{E}}\right), 2.89-2.91\left(2 \mathrm{H}, \mathrm{H}-6 \mathrm{~b}^{\mathrm{A}, \mathrm{D} \text { or B,E}}\right), 3.17$ (s, $\left.3 \mathrm{H}, \mathrm{OMe}\right), 3.22$ (s, $3 \mathrm{H}, \mathrm{OMe}), 3.27$ (s, $3 \mathrm{H}, \mathrm{OMe}), 3.44$ (s, $3 \mathrm{H}, \mathrm{OMe}), 3.45$ (s, $6 \mathrm{H}, \mathrm{OMe}), 3.45$ (s, $3 \mathrm{H}$, OMe), 3.46 (s, $3 \mathrm{H}, \mathrm{OMe}$ ), 3.53, (s, $6 \mathrm{H}, \mathrm{OMe}$ ), 3.57 (s, $3 \mathrm{H}, \mathrm{OMe}$ ), 3.59 (s, $3 \mathrm{H}, \mathrm{OMe}$ ), 3.62 (s, $3 \mathrm{H}, \mathrm{OMe}), 3.64$ (s, $6 \mathrm{H}, \mathrm{OMe}), 3.67$ (s, $3 \mathrm{H}, \mathrm{OMe}$ ), 3.67 (s, $3 \mathrm{H}, \mathrm{OMe}$ ), 3.10-4.27 (34 H, H-2, H-3, H-4, H-5 $\left.5^{\text {B,E or A,D }}, \mathrm{H}^{\mathrm{C}, \mathrm{F}, \mathrm{G}}, \mathrm{H}-6 \mathrm{~b}^{\mathrm{B}, \mathrm{E} \text { or A,D }}, \mathrm{H}-6^{\mathrm{C}, \mathrm{F}, \mathrm{G}}\right), 4.60-4.62\left(2 \mathrm{H}, \mathrm{H}-5^{\mathrm{A}, \mathrm{D} \text { or B,E }}\right)$, 
$4.91\left(\mathrm{~d}, 1 \mathrm{H},{ }^{3} J_{\mathrm{H}-1, \mathrm{H}-2}=3.8 \mathrm{~Hz}, \mathrm{H}-1\right), 4.94\left(\mathrm{~d}, 2 \mathrm{H},{ }^{3} J_{\mathrm{H}-1, \mathrm{H}-2}=3.3 \mathrm{~Hz}, \mathrm{H}-1\right), 4.97-5.00(3 \mathrm{H}, \mathrm{H}-$ 1), $5.11\left(\mathrm{~d}, 1 \mathrm{H},{ }^{3} J_{\mathrm{H}-1, \mathrm{H}-2}=3.6 \mathrm{~Hz}, \mathrm{H}-1\right), 7.46-7.47(6 \mathrm{H}, m-\mathrm{H}, p-\mathrm{H}), 7.77-7.83(4 \mathrm{H}, o-\mathrm{H})$ ppm; ${ }^{13} \mathrm{C}\left\{{ }^{1} \mathrm{H}\right\} \operatorname{NMR}\left(75.5 \mathrm{MHz} \mathrm{CDCl}_{3}, 25^{\circ} \mathrm{C}\right): \delta$ (assignment by HMQC) $=27.21\left(\mathrm{~d},{ }^{1} J_{\mathrm{C}, \mathrm{P}}=\right.$ $35.3 \mathrm{~Hz}), 27.91\left(\mathrm{~d},{ }^{1} J_{\mathrm{C}, \mathrm{P}}=34.4 \mathrm{~Hz}\right)\left(\mathrm{C}-6^{\mathrm{A}, \mathrm{D} \text { or B,E}}\right), 34.25\left(\mathrm{~d},{ }^{1} J_{\mathrm{C}, \mathrm{P}}=30.0 \mathrm{~Hz}\right), 35.15\left(\mathrm{~d},{ }^{1} J_{\mathrm{C}, \mathrm{P}}=\right.$ 30. Hz) $\left(\mathrm{C}-6^{\mathrm{B}, \mathrm{E} \text { or A,D}}\right), 57.91[\times 3], 58.00,58.21,58.45,58.49,58.66,58.82,58.90,61.45$, $61.57[\times 2], 61.82,61.91,62.20,62.22(\mathrm{OMe}), 64.52,64.74\left(\mathrm{C}-5^{\mathrm{A}, \mathrm{D}}\right.$ or B,E $), 68.67\left(\mathrm{~d},{ }^{2} J_{\mathrm{C}, \mathrm{P}}=6.4\right.$ $\mathrm{Hz}), 68.99\left(\mathrm{~d},{ }^{2} J_{\mathrm{C}, \mathrm{P}}=5.5 \mathrm{~Hz}\right)\left(\mathrm{C}-5^{\mathrm{B}, \mathrm{E}}\right.$ or A,D $), 70.86,71.05,71.44\left(\mathrm{C}-5^{\mathrm{C}, \mathrm{F}, \mathrm{G}}\right), 70.57,71.15,71.42$ $\left(\mathrm{C}-6^{\mathrm{C}, \mathrm{F}, \mathrm{G}}\right), 80.06,80.44,80.48,81.16,81.28,81.54,81.80,82.03,82.26$ [×5], 82.38, 82.46, $83.14,83.30\left(\mathrm{C}-2, \mathrm{C}-3, \mathrm{C}-4^{\mathrm{C}, \mathrm{F}, \mathrm{G}}\right), 86.73\left(\mathrm{~d},{ }^{3} J_{\mathrm{C}, \mathrm{P}}=10.1 \mathrm{~Hz}\right), 86.98\left(\mathrm{~d},{ }^{3} J_{\mathrm{C}, \mathrm{P}}=10.1 \mathrm{~Hz}\right)\left(\mathrm{C}-4^{\mathrm{A}, \mathrm{D}}\right.$ or B,E $), 89.09,89.46\left(\mathrm{C}-4^{\mathrm{B}, \mathrm{E} \text { or A,D }}\right), 98.46,98.54,99.55,100.28,100.45,100.78,101.18(\mathrm{C}-1)$, 128.73, $128.85(m-\mathrm{C}), 131.12,131.19(p-\mathrm{C}), 131.55\left(\mathrm{~d},{ }^{2} J_{\mathrm{C}-\mathrm{P}}=3.7 \mathrm{~Hz}\right), 131.67\left(\mathrm{~d},{ }^{2} J_{\mathrm{C}-\mathrm{P}}=3.7\right.$ $\mathrm{Hz})(o-\mathrm{C}), 131.34\left(\mathrm{~d},{ }^{1} J_{\mathrm{C}-\mathrm{P}}=11.4 \mathrm{~Hz}\right), 132.17\left(\mathrm{~d},{ }^{1} J_{\mathrm{C}-\mathrm{P}}=11.4 \mathrm{~Hz}\right)\left(\right.$ ipso-C) $\mathrm{ppm} ;{ }^{31} \mathrm{P}\left\{{ }^{1} \mathrm{H}\right\}$ NMR (121.5 MHz $\mathrm{CDCl}_{3}, 25^{\circ} \mathrm{C}$ ): $\delta=17.8$ (s), 18.4 (s) ppm; elemental analysis (\%) calcd for $\mathrm{C}_{71} \mathrm{H}_{116} \mathrm{~B}_{2} \mathrm{O}_{31} \mathrm{P}_{2} \cdot \mathrm{MeOH}(1549.23$ + 32.04): C 54.69, H 7.65, found: C 54.56, H 7.69; MS (ESI-TOF): $m / z(\%): 1571.71(100)[M+\mathrm{Na}]^{+}$.

$6^{\mathrm{A}}, 6^{\mathrm{B}}, 6^{\mathrm{D}}, 6^{\mathrm{E}}-$ Tetradeoxy- $6^{\mathrm{A}}, 6^{\mathrm{B}}: 6^{\mathrm{D}}, 6^{\mathrm{E}}-$ bis $[(R)$-phenylphosphinidene $]-2^{\mathrm{A}}, 2^{\mathrm{B}}, 2^{\mathrm{C}}, 2^{\mathrm{D}}, 2^{\mathrm{E}}, 2^{\mathrm{F}}, 2^{\mathrm{G}}$, $3^{\mathrm{A}}, 3^{\mathrm{B}}, 3^{\mathrm{C}}, 3^{\mathrm{D}}, 3^{\mathrm{E}}, 3^{\mathrm{F}}, 3^{\mathrm{G}}, 6^{\mathrm{C}}, 6^{\mathrm{F}}, 6^{\mathrm{G}}$-heptadeca- $O$-methyl- $\beta$-cyclodextrin (6)

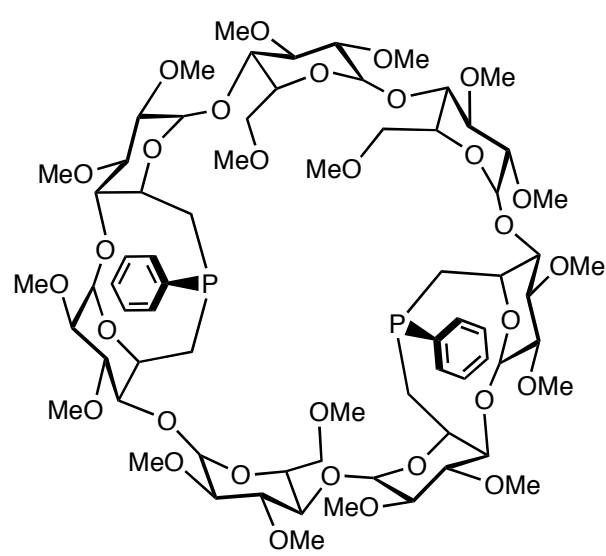

A solution of $5(80 \mathrm{mg}, 0.52 \mathrm{mmol})$ in $\mathrm{HNEt}_{2}(8 \mathrm{~mL})$ was refluxed for $12 \mathrm{~h}$. After cooling down to room temperature, the suspension was fitered over celite and the filtrate evaporated to dryness in vacuo to afford analytically pure $6(0.80 \mathrm{mg}, 99 \%) . R_{\mathrm{f}}\left(\mathrm{SiO}_{2}, \mathrm{CH}_{2} \mathrm{Cl}_{2} / \mathrm{MeOH}\right.$, 92:8, v/v) $=0.40$; m.p. $195^{\circ} \mathrm{C} ;{ }^{1} \mathrm{H}$ NMR $\left(300.1 \mathrm{MHz}, \mathrm{CDCl}_{3}, 25^{\circ} \mathrm{C}\right): \delta$ (assignment by COSY) $=1.73\left(\mathrm{~m}, 2 \mathrm{H}, \mathrm{H}-6 \mathrm{a}^{\mathrm{A}, \mathrm{D} \text { or B,E}}\right), 1.87\left(\mathrm{~m}, 2 \mathrm{H}, \mathrm{H}-6 \mathrm{a}^{\mathrm{B}, \mathrm{E} \text { or A,D }}\right), 2.81\left(\mathrm{td}, 2 \mathrm{H},{ }^{2} J_{\mathrm{H}-6 \mathrm{~b}, \mathrm{P}}={ }^{2} J_{\mathrm{H}-6 \mathrm{~b}, \mathrm{H}-6 \mathrm{a}}=\right.$ 
$\left.13.5 \mathrm{~Hz},{ }^{3} J_{\mathrm{H}-6 \mathrm{~b}, \mathrm{H}-5}=3.9 \mathrm{~Hz}, \mathrm{H}-6 \mathrm{~b}^{\mathrm{A}, \mathrm{D} \text { or B,E }}\right), 3.05-3.29\left(12 \mathrm{H}, \mathrm{H}-2, \mathrm{H}-6 \mathrm{a}^{\mathrm{C}, \mathrm{F}, \mathrm{G}}, \mathrm{H}-6 \mathrm{~b}^{\mathrm{B}, \mathrm{E}}\right.$ or A,D$)$, 3.11 (s, 3 H, OMe), 3.20 (s, 3 H, OMe), 3.27 (s, 3 H, OMe), 3.30-3.72 (14 H, H-3, H-4), 3.47 (s, $3 \mathrm{H}, \mathrm{OMe}), 3.48$ (s, $3 \mathrm{H}, \mathrm{OMe}), 3.49$ (s, $3 \mathrm{H}, \mathrm{OMe}), 3.50$ (s, $6 \mathrm{H}, \mathrm{OMe}), 3.55$ (s, $6 \mathrm{H}$, OMe), 3.60 (s, 3 H, OMe), 3.62 (s, 3 H, OMe), 3.65 (s, 9 H, OMe), 3.66 (s, 6 H, OMe), 3.81$3.42\left(8 \mathrm{H}, \mathrm{H}-5^{\mathrm{A}, \mathrm{D} \text { or B,E }}, \mathrm{H}-5^{\mathrm{C}, \mathrm{F}, \mathrm{G}}, \mathrm{H}-6 \mathrm{~b}^{\mathrm{C}, \mathrm{F}, \mathrm{G}}\right), 4.30$ (m, $\left.2 \mathrm{H}, \mathrm{H}-5^{\mathrm{B}, \mathrm{E} \text { or A,D }}\right), 4.95-5.05$ (6 H, H-1), $5.22\left(\mathrm{~d}, 1 \mathrm{H},{ }^{3} J_{\mathrm{H}-1, \mathrm{H}-2}=3.7 \mathrm{~Hz}, \mathrm{H}-1\right), 7.20-7.28(6 \mathrm{H}, m-\mathrm{H}, p-\mathrm{H}), 7.41-7.51(4 \mathrm{H}, o-\mathrm{H}) \mathrm{ppm}$; ${ }^{13} \mathrm{C}\left\{{ }^{1} \mathrm{H}\right\}$ NMR $\left(125.8 \mathrm{MHz} \mathrm{C}_{6} \mathrm{D}_{6}, 25^{\circ} \mathrm{C}\right): \delta$ (assignment by HMQC) $=28.66\left(\mathrm{~d},{ }^{1} J_{\mathrm{C}, \mathrm{P}}=15.4\right.$ $\mathrm{Hz}), 28.69\left(\mathrm{~d},{ }^{1} J_{\mathrm{C}, \mathrm{P}}=16.2 \mathrm{~Hz}\right)\left(\mathrm{C}-6^{\mathrm{A}, \mathrm{D}}\right.$ or B,E $), 34.99\left(\mathrm{~d},{ }^{1} J_{\mathrm{C}, \mathrm{P}}=19.1 \mathrm{~Hz}\right), 35.06\left(\mathrm{~d},{ }^{1} J_{\mathrm{C}, \mathrm{P}}=19.7\right.$ $\mathrm{Hz})\left(\mathrm{C}-6^{\mathrm{B}, \mathrm{E} \text { or A,D }}\right), 58.14[\times 2], 58.20,58.32,58.71,59.10,59.14,59.44,59.68,59.74,61.77$, $61.79,61.90,62.00,62.05,62.25[\times 2](\mathrm{OMe}), 67.54\left(\mathrm{~d},{ }^{2} J_{\mathrm{C}, \mathrm{P}}=11.7 \mathrm{~Hz}, \mathrm{C}-5^{\mathrm{A} \text { or D }}\right), 67.57(\mathrm{~d}$, $\left.{ }^{2} J_{\mathrm{C}, \mathrm{P}}=11.7 \mathrm{~Hz}, \mathrm{C}-5^{\mathrm{D} \text { or A}}\right), 71.53[\times 2], 71.80\left(\mathrm{C}-5^{\mathrm{C}, \mathrm{F}, \mathrm{G}}\right), 72.13\left(\mathrm{~d},{ }^{\mathrm{TS}} J_{\mathrm{C}, \mathrm{P}}=2.6 \mathrm{~Hz}, \mathrm{C}-6^{\mathrm{C}}\right), 72.39$ [×2] $\left(\mathrm{C}-6^{\mathrm{F}, \mathrm{G}}\right), 73.95\left(\mathrm{~d},{ }^{2} J_{\mathrm{C}, \mathrm{P}}=14.3 \mathrm{~Hz}, \mathrm{C}-5^{\mathrm{B} \text { or E}}\right), 74.26\left(\mathrm{~d},{ }^{2} J_{\mathrm{C}, \mathrm{P}}=13.6 \mathrm{~Hz}, \mathrm{C}-5^{\mathrm{E} \text { or B}}\right), 79.75$, $82.22,82.31,82.37,82.42,82.97,83.15,83.23,83.36$ [×3], 83.42, 83.46, 83.58, 84.07, 84.55, $84.70\left(\mathrm{C}-2, \mathrm{C}-3, \mathrm{C}-4^{\mathrm{C}, \mathrm{F}, \mathrm{G}}\right), 87.15\left(\mathrm{~d},{ }^{3} J_{\mathrm{C}, \mathrm{P}}=8.0 \mathrm{~Hz}, \mathrm{C}-4^{\mathrm{B} \text { or E}}\right), 87.32\left(\mathrm{~d},{ }^{3} J_{\mathrm{C}, \mathrm{P}}=8.0 \mathrm{~Hz}, \mathrm{C}-4^{\mathrm{E} \text { or }}\right.$ B), $90.23\left(\mathrm{~d},{ }^{3} J_{\mathrm{C}, \mathrm{P}}=2.7 \mathrm{~Hz}, \mathrm{C}-4^{\mathrm{A} \text { or D }}\right), 90.38\left(\mathrm{~d},{ }^{3} J_{\mathrm{C}, \mathrm{P}}=2.6 \mathrm{~Hz}, \mathrm{C}-4^{\mathrm{D}}\right.$ or A $), 99.27,99.32,99.69$, 99.90, 99.96 [×2], $101.19(\mathrm{C}-1), 128.96,129.03(p-\mathrm{C}), 129.15$ [×2] (overlapping d, ${ }^{3} J_{\mathrm{C}, \mathrm{P}}=4.8$ $\mathrm{Hz}, m-\mathrm{C}), 132.47\left(\mathrm{~d},{ }^{2} J_{\mathrm{C}, \mathrm{P}}=18.7 \mathrm{~Hz}, o-\mathrm{C}\right), 132.51\left(\mathrm{~d},{ }^{2} J_{\mathrm{C}, \mathrm{P}}=18.7 \mathrm{~Hz}, o-\mathrm{C}\right), 142.33[\times 2](\mathrm{d}$, ${ }^{1} J_{\mathrm{C}, \mathrm{P}}=11.7 \mathrm{~Hz}$, ipso-C) ppm; ${ }^{31} \mathrm{P}\left\{{ }^{1} \mathrm{H}\right\}$ NMR $\left(202.5 \mathrm{MHz} \mathrm{C}_{6} \mathrm{D}_{6}, 25{ }^{\circ} \mathrm{C}\right): \delta=-15.0(\mathrm{~s}),-15.2$ (s) ppm; elemental analysis (\%) calcd for $\mathrm{C}_{71} \mathrm{H}_{110} \mathrm{O}_{31} \mathrm{P}_{2} \cdot 0.5 \mathrm{CH}_{2} \mathrm{Cl}_{2}(1521.56+42.47)$ : $\mathrm{C}$ 54.91, H 7.15, found: C 54.82, H 7.35; MS (ESI-TOF): $m / z$ (\%): 1521.57 (100) $[M+\mathrm{H}]^{+}$.

$6^{\mathrm{A}}, 6^{\mathrm{B}}, 6^{\mathrm{D}}, 6^{\mathrm{E}}-$ Tetradeoxy- $6^{\mathrm{A}}, 6^{\mathrm{B}}: 6^{\mathrm{D}}, 6^{\mathrm{E}}-$ bis $[(S)$-phenyloxophosphinidene $]-2^{\mathrm{A}}, 2^{\mathrm{B}}, 2^{\mathrm{C}}, 2^{\mathrm{D}}, 2^{\mathrm{E}}, 2^{\mathrm{F}}$, $2^{\mathrm{G}}, 3^{\mathrm{A}}, 3^{\mathrm{B}}, 3^{\mathrm{C}}, 3^{\mathrm{D}}, 3^{\mathrm{E}}, 3^{\mathrm{F}}, 3^{\mathrm{G}}, 6^{\mathrm{C}}, 6^{\mathrm{F}}, 6^{\mathrm{G}}$-heptadeca- $O$-methyl- $\beta$-cyclodextrin (7)

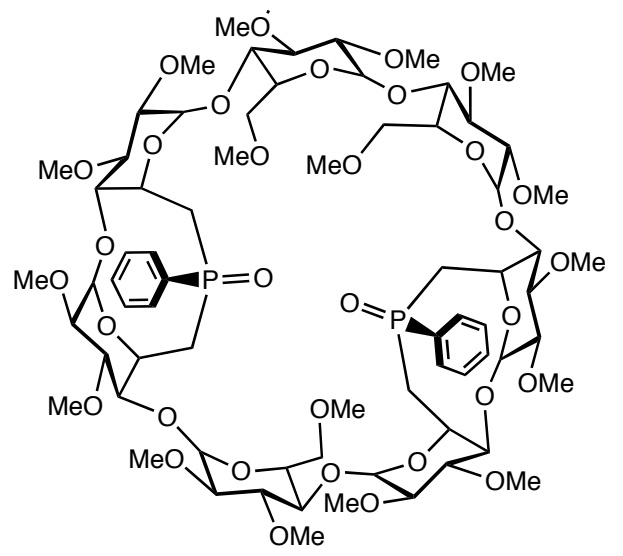


The bis(phosphane oxyde) 7 was quantitavely obtained by bubbling air through a solution of 6 in $\mathrm{MeOH}$ for $3 \mathrm{~h}$ at room temperature. Removal of the solvent in vacuo gave 7 as a white analytically pure product. $R_{\mathrm{f}}\left(\mathrm{SiO}_{2}, \mathrm{CH}_{2} \mathrm{Cl}_{2} / \mathrm{MeOH}, 92: 8, v / v\right)=0.30 ;$ m.p. $213^{\circ} \mathrm{C} ;{ }^{1} \mathrm{H} \mathrm{NMR}$ $\left(300.1 \mathrm{MHz}, \mathrm{CDCl}_{3}, 25^{\circ} \mathrm{C}\right): \delta$ (assignment by COSY $)=1.91-1.93\left(2 \mathrm{H}, \mathrm{H}-6 \mathrm{a}^{\mathrm{A}, \mathrm{D} \text { or B,E }}\right), 2.21-$ $2.23\left(2 \mathrm{H}, 6 \mathrm{a}^{\mathrm{B}, \mathrm{E}}\right.$ or A,D $), 2.91-3.74\left(28 \mathrm{H}, \mathrm{H}-2, \mathrm{H}-3, \mathrm{H}-4, \mathrm{H}-6 \mathrm{~b}^{\mathrm{A}, \mathrm{B}, \mathrm{D}, \mathrm{E}}, \mathrm{H}-6 \mathrm{a}^{\mathrm{C}, \mathrm{F}, \mathrm{G}}\right), 3.06(\mathrm{~s}, 3 \mathrm{H}$, OMe), 3.08 (s, 3 H, OMe), 3.22 (s, 3 H, OMe), 3.43 (s, 3 H, OMe), 3.44 (s, 3 H, OMe), 3.45 (s, $3 \mathrm{H}, \mathrm{OMe}), 3.47$ (s, $3 \mathrm{H}, \mathrm{OMe}), 3.48$ (s, $3 \mathrm{H}, \mathrm{OMe}), 3.56$ (s, $9 \mathrm{H}, \mathrm{OMe}), 3.60$ (s, $6 \mathrm{H}$, OMe), 3.67 (s, $6 \mathrm{H}, \mathrm{OMe}$ ), 3.68 (s, $3 \mathrm{H}, \mathrm{OMe}), 3.71$ (s, $3 \mathrm{H}, \mathrm{OMe}), 3.86-4.08$ (6 H, H-5 $5^{\mathrm{C}, \mathrm{F}, \mathrm{G}}$, $\left.\mathrm{H}-6 \mathrm{~b}^{\mathrm{C}, \mathrm{F}, \mathrm{G}}\right), 4.40-4.42\left(2 \mathrm{H}, \mathrm{H}-5^{\mathrm{A}, \mathrm{D} \text { or B,E }}\right), 4.62-4.64\left(2 \mathrm{H}, \mathrm{H}-5^{\mathrm{B}, \mathrm{E}}\right.$ or A,D$), 4.86\left(\mathrm{~d}, 1 \mathrm{H},{ }^{3} J_{\mathrm{H}-1, \mathrm{H}-2}=\right.$ $3.3 \mathrm{~Hz}, \mathrm{H}-1), 4.93\left(\mathrm{~d}, 2 \mathrm{H},{ }^{3} J_{\mathrm{H}-1, \mathrm{H}-2}=3.3 \mathrm{~Hz}, \mathrm{H}-1\right), 4.97$ (d, $\left.1 \mathrm{H},{ }^{3} J_{\mathrm{H}-1, \mathrm{H}-2}=3.3 \mathrm{~Hz}, \mathrm{H}-1\right), 5.02$ $\left(\mathrm{d}, 2 \mathrm{H},{ }^{3} J_{\mathrm{H}-1, \mathrm{H}-2}=3.3 \mathrm{~Hz}, \mathrm{H}-1\right), 5.08\left(\mathrm{~d}, 1 \mathrm{H},{ }^{3} J_{\mathrm{H}-1, \mathrm{H}-2}=3.3 \mathrm{~Hz}, \mathrm{H}-1\right), 7.45-7.54(6 \mathrm{H}, m-\mathrm{H}, p-$ $\mathrm{H}), 7.72-7.80(4 \mathrm{H}, o-\mathrm{H}) \mathrm{ppm} ;{ }^{13} \mathrm{C}\left\{{ }^{1} \mathrm{H}\right\} \mathrm{NMR}\left(75.5 \mathrm{MHz} \mathrm{CDCl}_{3}, 25^{\circ} \mathrm{C}\right): \delta$ (assignment by $\mathrm{HMQC})=33.41\left(\mathrm{~d},{ }^{2} J_{\mathrm{C}, \mathrm{P}}=68.1 \mathrm{~Hz}\right), 33.50\left(\mathrm{~d},{ }^{2} J_{\mathrm{C}, \mathrm{P}}=68.1 \mathrm{~Hz}\right)\left(\mathrm{C}-6^{\mathrm{A}, \mathrm{D} \text { or B,E}}\right), 37.82\left(\mathrm{~d},{ }^{2} J_{\mathrm{C}, \mathrm{P}}=\right.$ $65.5 \mathrm{~Hz}), 37.87\left(\mathrm{~d},{ }^{2} J_{\mathrm{C}, \mathrm{P}}=65.5 \mathrm{~Hz}\right)\left(\mathrm{C}-6^{\mathrm{B}, \mathrm{E} \text { or A,D}}\right), 57.89,58.14,58.17[\times 2], 58.21,58.25[\times 2]$, $58.66,58.73,58.81,61.48,61.50,61.62[\times 2], 61.93,62.10,62.22(\mathrm{OMe}), 63.33[\times 2]\left(\mathrm{C}-5^{\mathrm{A}, \mathrm{D}}\right.$ or $\mathrm{B}, \mathrm{E}), 66.22,66.83\left(\mathrm{C}-5^{\mathrm{B}, \mathrm{E}}\right.$ or A,D $), 70.30,70.74,70.83\left(\mathrm{C}-5^{\mathrm{C}, \mathrm{F}, \mathrm{G}}\right), 70.89,70.96,71.04(\mathrm{C}-$ $\left.6^{\mathrm{C}, \mathrm{F}, \mathrm{G}}\right), 80.31,80.57,81.25,81.50[\times 2], 81.67,81.85[\times 2], 82.04,82.41[\times 4], 82.65[\times 2]$, $83.37,83.57\left(\mathrm{C}-2, \mathrm{C}-3, \mathrm{C}-4^{\mathrm{C}, \mathrm{F}, \mathrm{G}}\right), 86.25\left(\mathrm{~d},{ }^{3} J_{\mathrm{C}, \mathrm{P}}=11.8 \mathrm{~Hz}\right), 87.21\left(\mathrm{~d},{ }^{3} J_{\mathrm{C}, \mathrm{P}}=11.8 \mathrm{~Hz}\right)\left(\mathrm{C}-4^{\mathrm{A}, \mathrm{D}}\right.$ or $\mathrm{B}, \mathrm{E}), 89.29[\times 2]\left(\mathrm{C}-4^{\mathrm{B}, \mathrm{E}}\right.$ or $\left.\mathrm{A}, \mathrm{D}\right), 98.81,98.94,99.73,100.35[\times 2], 100.65,101.15(\mathrm{C}-1)$, $128.71[\times 2]\left(\mathrm{d},{ }^{2} J_{\mathrm{C}, \mathrm{P}}=11.8 \mathrm{~Hz}, o-\mathrm{C}\right), 129.44[\times 2]\left(\mathrm{d},{ }^{3} J_{\mathrm{C}, \mathrm{P}}=9.3 \mathrm{~Hz}, m-\mathrm{C}\right), 131.69[\times 2](p-\mathrm{C})$, $135.16\left(\mathrm{~d},{ }^{1} J_{\mathrm{C}, \mathrm{P}}=98.1 \mathrm{~Hz}\right.$, ipso-C), $135.36\left(\mathrm{~d},{ }^{1} J_{\mathrm{C}, \mathrm{P}}=98.1 \mathrm{~Hz}\right.$, ipso-C) ppm; ${ }^{31} \mathrm{P}\left\{{ }^{1} \mathrm{H}\right\} \mathrm{NMR}$ (121.5 $\left.\mathrm{MHz} \mathrm{CDCl}_{3}, 25^{\circ} \mathrm{C}\right): \delta=35.7$ (s), 35.9 (s) ppm; elemental analysis (\%) calcd for $\mathrm{C}_{71} \mathrm{H}_{110} \mathrm{O}_{33} \mathrm{P}_{2}$ (1553.56): C 54.89, H 7.14, found: C 54.82, H 7.53; MS (ESI-TOF): $\mathrm{m} / z$ (\%): $1575.56(100)[M+\mathrm{Na}]^{+}$.

$P, P^{\prime}-\left\{6^{\mathrm{A}}, 6^{\mathrm{B}}, 6^{\mathrm{D}}, 6^{\mathrm{E}}-\right.$ Tetradeoxy-6 ${ }^{\mathrm{A}}, 6^{\mathrm{B}}: 6^{\mathrm{D}}, 6^{\mathrm{E}}$-bis $[(R)$-phenylphosphinidene $]-2^{\mathrm{A}}, 2^{\mathrm{B}}, 2^{\mathrm{C}}, 2^{\mathrm{D}}, 2^{\mathrm{E}}$, $2^{\mathrm{F}}, 2^{\mathrm{G}}, 3^{\mathrm{A}}, 3^{\mathrm{B}}, 3^{\mathrm{C}}, 3^{\mathrm{D}}, 3^{\mathrm{E}}, 3^{\mathrm{F}}, 3^{\mathrm{G}}, 6^{\mathrm{C}}, 6^{\mathrm{F}}, 6^{\mathrm{G}}$-heptadeca- $O$-methyl- $\beta$-cyclodextrin $\}$ gold(I) hexafluorophosphate (8) 


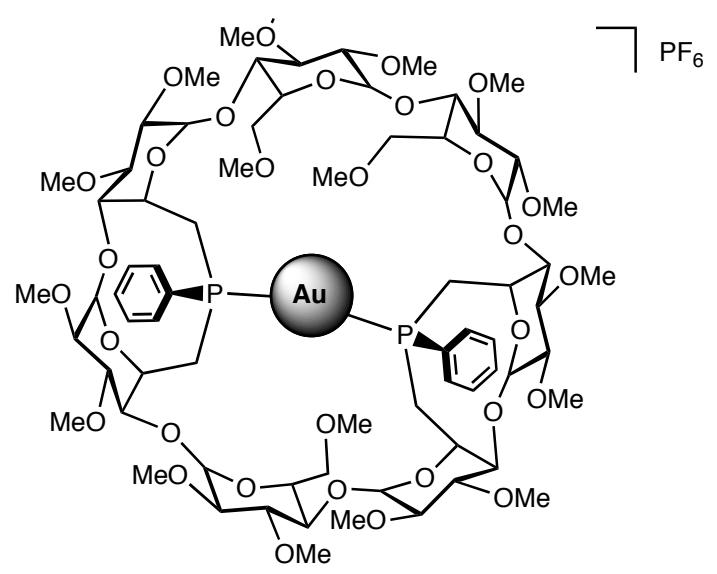

A solution of thallium hexafluorophosphate $(0.020 \mathrm{~g}, 0.058 \mathrm{mmol})$ in THF $(2 \mathrm{~mL})$ was added to a solution of $[\mathrm{AuCl}(\mathrm{THT})]\left(\mathrm{THT}=\right.$ tetrathydrothiophene) $(0.016 \mathrm{~g}, 0.053 \mathrm{mmol})$ in $\mathrm{CH}_{2} \mathrm{Cl}_{2}$ $(10 \mathrm{~mL})$. After stirring for $30 \mathrm{~min}$, the solution was filtered through celite to eliminate thallium chloride, then added to a solution of $6(0.080 \mathrm{~g}, 0.053 \mathrm{mmol})$ in $\mathrm{CH}_{2} \mathrm{Cl}_{2}(5 \mathrm{~mL})$. After $30 \mathrm{~min}$, the solution was filtered through celite and the filtered solution was concentrated to $c a .2 \mathrm{~mL}$. Addition of pentane afforded complex $8(0.099 \mathrm{~g}, 99 \%)$ as a pale brown precipitate. $R_{\mathrm{f}}\left(\mathrm{SiO}_{2}, \mathrm{CH}_{2} \mathrm{Cl}_{2} / \mathrm{MeOH}, 92: 8, v / v\right)=0.33$; m.p. dec.; ${ }^{1} \mathrm{H}$ NMR $(300.1$ $\left.\mathrm{MHz}, \mathrm{CDCl}_{3}, 25^{\circ} \mathrm{C}\right): \delta$ (assignment by COSY) $=2.06\left(\mathrm{dt}, 1 \mathrm{H},{ }^{2} J_{\mathrm{H}-6 \mathrm{a}, \mathrm{H}-6 \mathrm{~b}}=15.4 \mathrm{~Hz},{ }^{2} J_{\mathrm{H}-6 \mathrm{a}, \mathrm{P}}=\right.$ ${ }^{3} J_{\mathrm{H}-6 \mathrm{a}, \mathrm{H}-65}=5.7 \mathrm{~Hz}, \mathrm{H}-6 \mathrm{a}^{\mathrm{A}}$ or D $), 2.52\left(\mathrm{~m}, 1 \mathrm{H}, \mathrm{H}-6 \mathrm{a}^{\mathrm{D}}\right.$ or A $), 2.65\left(2 \mathrm{H}, \mathrm{H}-6 \mathrm{a}^{\mathrm{B}, \mathrm{E}}\right), 2.84(\mathrm{~s}, 3 \mathrm{H}$, OMe), 2.95-3.70 (30 H, H-2, H-3, H-4 ${ }^{\mathrm{A}, \mathrm{B}, \mathrm{D}, \mathrm{E} \text { and C,F or F,G or C,G }}, \mathrm{H}-5^{\mathrm{C} \text { or F or G }}, \mathrm{H}-6^{\mathrm{C}, \mathrm{F}}$ or F,G or C,G, $\mathrm{H}-$ $\left.6 \mathrm{a}^{\mathrm{G} \text { or } \mathrm{C} \text { or } \mathrm{F}}, \mathrm{H}-6 \mathrm{~b}^{\mathrm{A}, \mathrm{B}, \mathrm{D}, \mathrm{E}}\right), 2.96(\mathrm{~s}, 3 \mathrm{H}, \mathrm{OMe}), 3.30$ (s, $\left.3 \mathrm{H}, \mathrm{OMe}\right), 3.47$ (s, $\left.6 \mathrm{H}, \mathrm{OMe}\right), 3.50$ (s, 6 H, OMe), 3.52 (s, $3 \mathrm{H}, \mathrm{OMe}), 3.54$ (s, $3 \mathrm{H}, \mathrm{OMe}), 3.55$ (s, $3 \mathrm{H}, \mathrm{OMe}), 3.56$ (s, $3 \mathrm{H}, \mathrm{OMe})$, 3.60 (s, $3 \mathrm{H}, \mathrm{OMe}$ ), 3.61 (s, $6 \mathrm{H}, \mathrm{OMe}), 3.64$ (s, $6 \mathrm{H}, \mathrm{OMe}), 3.67$ (s, $3 \mathrm{H}, \mathrm{OMe}), 3.80-3.90$ (2 $\left.\mathrm{H}, \mathrm{H}-4^{\mathrm{G} \text { or } \mathrm{C} \text { or F}}, \mathrm{H}-6 \mathrm{~b}^{\mathrm{G} \text { or } \mathrm{C} \text { or F }}\right), 4.21-4.43\left(6 \mathrm{H}, \mathrm{H}-5^{\mathrm{A}, \mathrm{B}, \mathrm{D}, \mathrm{E} \text { and } \mathrm{F}, \mathrm{G} \text { or C,F or C,G }}\right), 4.83\left(\mathrm{~d}, 1 \mathrm{H},{ }^{3} J_{\mathrm{H}-1, \mathrm{H}-}\right.$ $\left.{ }_{2}=3.1 \mathrm{~Hz}, \mathrm{H}-1\right), 4.99\left(\mathrm{~d}, 1 \mathrm{H},{ }^{3} J_{\mathrm{H}-1, \mathrm{H}-2}=2.0 \mathrm{~Hz}, \mathrm{H}-1\right), 5.06\left(\mathrm{~d}, 1 \mathrm{H},{ }^{3} J_{\mathrm{H}-1, \mathrm{H}-2}=3.1 \mathrm{~Hz}, \mathrm{H}-1\right)$, $5.07\left(\mathrm{~d}, 1 \mathrm{H},{ }^{3} J_{\mathrm{H}-1, \mathrm{H}-2}=3.3 \mathrm{~Hz}, \mathrm{H}-1\right), 5.10\left(\mathrm{~d}, 1 \mathrm{H},{ }^{3} J_{\mathrm{H}-1, \mathrm{H}-2}=4.5 \mathrm{~Hz}, \mathrm{H}-1\right), 5.15\left(\mathrm{~d}, 1 \mathrm{H},{ }^{3} J_{\mathrm{H}-}\right.$ $1, \mathrm{H}-2=3.3 \mathrm{~Hz}, \mathrm{H}-1), 5.18\left(\mathrm{~d}, 1 \mathrm{H},{ }^{3} J_{\mathrm{H}-1, \mathrm{H}-2}=3.9 \mathrm{~Hz}, \mathrm{H}-1\right), 7.42-7.47(2 \mathrm{H}, m-\mathrm{H}), 7.55-7.67$ (6 $\mathrm{H}, m-\mathrm{H}, p-\mathrm{H}, o-\mathrm{H}), 7.73-7.79(2 \mathrm{H}, o-\mathrm{H}) \mathrm{ppm} ;{ }^{13} \mathrm{C}\left\{{ }^{1} \mathrm{H}\right\} \mathrm{NMR}\left(75.5 \mathrm{MHz}, \mathrm{CDCl}_{3}, 25^{\circ} \mathrm{C}\right): \delta$ $($ assignment by HMQC $)=28.27,28.96\left(\mathrm{C}-6^{\mathrm{B}, \mathrm{E}}\right.$ or A,D $), 36.23,38.66\left(\mathrm{C}-6^{\mathrm{A}, \mathrm{D}}\right.$ or B,E $), 57.77[\times 2]$, 58.14 [×2], 58.22, 58.27, 58.50, 58.80, 59.09, 59.29, 59.51, 59.59, 60.04, 60.96, 60.97 [×2], $61.40(\mathrm{OMe}), 64.40,65.05\left(\mathrm{C}-5^{\mathrm{A}, \mathrm{D} \text { or B,E}}\right), 69.52,70.43[\times 2]\left(\mathrm{C}-5^{\mathrm{B}, \mathrm{E}}\right.$ or A,D and $\mathrm{C}$ or F or $\left.\mathrm{G}\right), 72.64$, $74.03\left(\mathrm{C}-5^{\mathrm{F}, \mathrm{G} \text { or } \mathrm{C}, \mathrm{F} \text { or C,G}}\right), 71.26,71.95,72.77\left(\mathrm{C}-6^{\mathrm{C}, \mathrm{F}, \mathrm{G}}\right), 76.90,77.07,78.07\left(\mathrm{C}-4^{\mathrm{C}, \mathrm{F}, \mathrm{G}}\right), 80.29$, $80.67,81.23,81.28$ [×2], 81.70, 81.79 [×3], 81.84, 82.36 [×2], 82.78, 83.91 (C-2, C-3), 88.61 $[\times 2]\left(\mathrm{C}-4^{\mathrm{A}, \mathrm{B} \text { or } \mathrm{D}, \mathrm{E}}\right), 86.21[\times 2]\left(\mathrm{C}-4^{\mathrm{D}, \mathrm{E}}\right.$ or A,B $), 95.20,97.01,97.44,97.61,98.73,99.42,99.54$ (C-1), 129.59, $129.76(m-\mathrm{C}), 132.48$ [×2] (p-C), 132.30, 132.69 (o-C), 137.75 [×2] (ipso-C) 
ppm; ${ }^{31} \mathrm{P}\left\{{ }^{1} \mathrm{H}\right\}$ NMR $\left(121.5 \mathrm{MHz}, \mathrm{CDCl}_{3}, 25^{\circ} \mathrm{C}\right.$ ): $\delta=34.2$ (br s), -144.3 (hept, ${ }^{1} J_{\mathrm{P}, \mathrm{F}}=716 \mathrm{~Hz}$ ) ppm; ${ }^{31} \mathrm{P}\left\{{ }^{1} \mathrm{H}\right\}$ NMR $\left(121.5 \mathrm{MHz}, \mathrm{CD}_{2} \mathrm{Cl}_{2}, 25^{\circ} \mathrm{C}\right): \delta=37.6$ and 34.6 (AB system, ${ }^{2} J_{\mathrm{P} 1, \mathrm{P} 2}=326$ $\mathrm{Hz}),-144.3$ (hept, ${ }^{1} J_{\mathrm{P}, \mathrm{F}}=716 \mathrm{~Hz}$ ) ppm; ${ }^{31} \mathrm{P}\left\{{ }^{1} \mathrm{H}\right\} \mathrm{NMR}\left(121.5 \mathrm{MHz}, \mathrm{CD}_{2} \mathrm{Cl}_{2},-80^{\circ} \mathrm{C}\right): \delta=40.6$ and $31.6\left(\mathrm{AB}\right.$ system, $\left.{ }^{2} J_{\mathrm{P} 1, \mathrm{P} 2}=326 \mathrm{~Hz}\right), 38.0$ and $33.8\left(\mathrm{AB}\right.$ system, $\left.{ }^{2} J_{\mathrm{P} 1, \mathrm{P} 2}=326 \mathrm{~Hz}\right),-144.3$ (hept, ${ }^{1} J_{\mathrm{P}, \mathrm{F}}=716 \mathrm{~Hz}$ ) ppm; elemental analysis (\%) calcd for $\mathrm{C}_{71} \mathrm{H}_{110} \mathrm{AuF}_{6} \mathrm{O}_{31} \mathrm{P}_{3}$ (1863.49): C 45.76, H 5.95, found: C 45.76, H 5.95; MS (ESI-TOF): $m / z(\%): 1717.62(100)\left[M-\mathrm{PF}_{6}\right]^{+}$.

trans- $P, P$ '-Dichlorido- $\left\{6^{\mathrm{A}}, 6^{\mathrm{B}}, 6^{\mathrm{D}}, 6^{\mathrm{E}}\right.$-tetradeoxy- $6^{\mathrm{A}}, 6^{\mathrm{B}}: 6^{\mathrm{D}}, 6^{\mathrm{E}}$-bis $[(R)$-phenylphosphinidene $]-$ $2^{\mathrm{A}}, 2^{\mathrm{B}}, 2^{\mathrm{C}}, 2^{\mathrm{D}}, 2^{\mathrm{E}}, 2^{\mathrm{F}}, 2^{\mathrm{G}}, 3^{\mathrm{A}}, 3^{\mathrm{B}}, 3^{\mathrm{C}}, 3^{\mathrm{D}}, 3^{\mathrm{E}}, 3^{\mathrm{F}}, 3^{\mathrm{G}}, 6^{\mathrm{C}}, 6^{\mathrm{F}}, 6^{\mathrm{G}}$-heptadeca- $O$-methyl- $\beta$ cyclodextrin $\}$ palladium(II) (9)

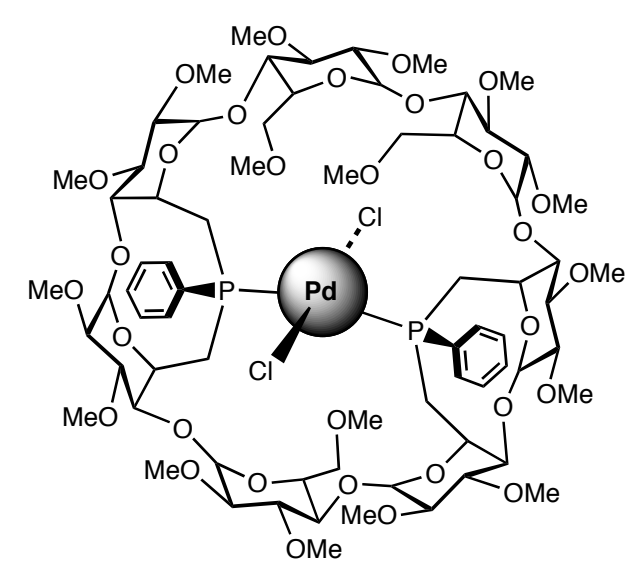

A solution of $\left[\mathrm{PdCl}_{2}(\mathrm{PhCN})_{2}\right](0.020 \mathrm{~g}, 0.053 \mathrm{mmol})$ in $\mathrm{CH}_{2} \mathrm{Cl}_{2}(5 \mathrm{~mL})$ was added dropwise to a solution of diphosphine $6(0.080 \mathrm{~g}, 0.053 \mathrm{mmol})$ in $\mathrm{CH}_{2} \mathrm{Cl}_{2}(10 \mathrm{~mL})$ within $30 \mathrm{~min}$ at room temperature. After $30 \mathrm{~min}$, the reaction mixture was evaporated to dryness and the residue subjected to column chromatography $\left(\mathrm{SiO}_{2}, \mathrm{CH}_{2} \mathrm{Cl}_{2} / \mathrm{MeOH}, 96: 4, v / v\right)$ to give pure 9 $(0.081 \mathrm{~g}, 90 \%)$ as a pale yellow powder. $R_{\mathrm{f}}\left(\mathrm{SiO}_{2}, \mathrm{CH}_{2} \mathrm{Cl}_{2} / \mathrm{MeOH}, 92: 8, v / v\right)=0.40$; m.p. dec.; ${ }^{1} \mathrm{H}$ NMR $\left(300.1 \mathrm{MHz}, \mathrm{CD}_{2} \mathrm{Cl}_{2}, 25^{\circ} \mathrm{C}\right): \delta$ (assignment by COSY) $=2.0\left(\mathrm{~m}, 1 \mathrm{H}, \mathrm{H}-6 \mathrm{a}^{\mathrm{A}}\right.$ or D), $2.29\left(\mathrm{~m}, 1 \mathrm{H}, \mathrm{H}-6 \mathrm{a}^{\mathrm{D} \text { or A }}\right), 2.55\left(\mathrm{~m}, 1 \mathrm{H}, \mathrm{H}-6 \mathrm{a}^{\mathrm{B} \text { or E}}\right), 2.70\left(\mathrm{~m}, 1 \mathrm{H}, \mathrm{H}-6 \mathrm{a}^{\mathrm{E}}\right.$ or B$), 2.78(\mathrm{dd}, 1 \mathrm{H}$, $\left.{ }^{3} J_{\mathrm{H}-2, \mathrm{H}-3}=9.6 \mathrm{~Hz},{ }^{3} J_{\mathrm{H}-2, \mathrm{H}-1}=3.5 \mathrm{~Hz}, \mathrm{H}-2\right), 2.98-3.14\left(\mathrm{~m}, 8 \mathrm{H}, \mathrm{H}-2, \mathrm{H}-4^{\mathrm{D}, \mathrm{E}}\right), 3.25-3.88(\mathrm{~m}, 20$ $\left.\mathrm{H}, \mathrm{H}-3, \mathrm{H}-4^{\mathrm{A}, \mathrm{B}, \mathrm{C}, \mathrm{F}, \mathrm{G}}, \mathrm{H}-6 \mathrm{a}^{\mathrm{C}, \mathrm{F}, \mathrm{G}}, \mathrm{H}-6 \mathrm{~b}^{\mathrm{A}, \mathrm{B}, \mathrm{D}, \mathrm{E} \text { and } \mathrm{C} \text { or } \mathrm{F} \text { or } \mathrm{G}}\right), 3.22(\mathrm{~s}, 3 \mathrm{H}, \mathrm{OMe}), 3.24(\mathrm{~s}, 3 \mathrm{H}$, OMe), 3.25 (s, 3 H, OMe), 3.35 (s, 3 H, OMe), 3.43 (s, 3 H, OMe), 3.44 (s, 3 H, OMe), 3.46 (s, $3 \mathrm{H}, \mathrm{OMe}), 3.47$ (s, $3 \mathrm{H}, \mathrm{OMe}), 3.51(\mathrm{~s}, 3 \mathrm{H}, \mathrm{OMe}), 3.54$ (s, $6 \mathrm{H}, \mathrm{OMe}), 3.55$ (s, $6 \mathrm{H}$, OMe), 3.57 (s, 3 H, OMe), 3.59 (s, 3 H, OMe), 3.62 (s, 3 H, OMe), 3.73 (s, 3 H, OMe), 3.97$4.11\left(\mathrm{~m}, 3 \mathrm{H}, \mathrm{H}-5^{\mathrm{C}}\right.$ or F or G, $\left.\mathrm{H}-6 \mathrm{~b}^{\mathrm{C}, \mathrm{F} \text { or } \mathrm{C}, \mathrm{G} \text { or F,G }}\right), 4.20-4.33\left(\mathrm{~m}, 4 \mathrm{H}, \mathrm{H}-5^{\mathrm{A}, \mathrm{B} \text { and } \mathrm{F}, \mathrm{G} \text { or C,F or C,G }}\right)$, $4.36-4.41\left(\mathrm{~m}, 1 \mathrm{H}, \mathrm{H}-5^{\mathrm{E}}\right.$ or D $), 4.44-4.90\left(\mathrm{~m}, 1 \mathrm{H}, \mathrm{H}-5^{\mathrm{D} \text { or E}}\right), 4.81\left(\mathrm{~d}, 1 \mathrm{H},{ }^{3} J_{\mathrm{H}-1, \mathrm{H}-2}=3.6 \mathrm{~Hz}, \mathrm{H}-\right.$ 
1), $4.91\left(\mathrm{~d}, 1 \mathrm{H},{ }^{3} J_{\mathrm{H}-1, \mathrm{H}-2}=3.4 \mathrm{~Hz}, \mathrm{H}-1\right), 4.99\left(\mathrm{~d}, 1 \mathrm{H},{ }^{3} J_{\mathrm{H}-1, \mathrm{H}-2}=3.1 \mathrm{~Hz}, \mathrm{H}-1\right), 5.00(\mathrm{~d}, 1 \mathrm{H}$, $\left.{ }^{3} J_{\mathrm{H}-1, \mathrm{H}-2}=3.3 \mathrm{~Hz}, \mathrm{H}-1\right), 5.01\left(\mathrm{~d}, 1 \mathrm{H},{ }^{3} J_{\mathrm{H}-1, \mathrm{H}-2}=3.1 \mathrm{~Hz}, \mathrm{H}-1\right), 5.20\left(\mathrm{~d}, 1 \mathrm{H},{ }^{3} J_{\mathrm{H}-1, \mathrm{H}-2}=4.0 \mathrm{~Hz}\right.$, $\mathrm{H}-1), 5.40\left(\mathrm{~d}, 1 \mathrm{H},{ }^{3} J_{\mathrm{H}-1, \mathrm{H}-2}=4.4 \mathrm{~Hz}, \mathrm{H}-1\right), 7.36-7.42$ (m, $\left.6 \mathrm{H}, m-\mathrm{H}, p-\mathrm{H}\right), 7.95-8,01$ (m, $4 \mathrm{H}$, $o$-H) ppm; ${ }^{13} \mathrm{C}\left\{{ }^{1} \mathrm{H}\right\}$ NMR $\left(75.5 \mathrm{MHz}, \mathrm{CD}_{2} \mathrm{Cl}_{2}, 25^{\circ} \mathrm{C}\right): \delta$ (assignment by HMQC) $=22.86$, $25.06\left(\mathrm{C}-6^{\mathrm{A}, \mathrm{D}}\right.$ or B,E $), 31.51,32.00\left(\mathrm{C}-6^{\mathrm{B}, \mathrm{E}}\right.$ or A,D $), 54.74,55.93,55.88[\times 2], 56.50,56.56[\times 2]$, $56.74,57.64,58.09,58.37,58.45,58.86$ [×2], $59.27[\times 2], 59.45(\mathrm{OMe}), 61.71,62.31\left(\mathrm{C}-5^{\mathrm{A}, \mathrm{D}}\right.$ or $\left.{ }^{\mathrm{B}, \mathrm{E}}\right), 66.18,67.25\left(\mathrm{C}-5^{\mathrm{B}, \mathrm{E} \text { or A,D }}\right), 68.58,68.73,68.87\left(\mathrm{C}-5^{\mathrm{C}, \mathrm{F}, \mathrm{G}}\right), 69.07,69.84,70.03(\mathrm{C}-$ $\left.6^{\mathrm{C}, \mathrm{F}, \mathrm{G}}\right), 75.71,75.83[\times 2]\left(\mathrm{C}-4^{\mathrm{C}, \mathrm{F}, \mathrm{G}}\right), 77.25,77.45,78.64,79.08,79.16,79.33,79.39,79.47$ [×2], 79.74, 80.12, 80.45, 80.96 [×2] (C-2, C-3), $82.50[\times 2], 87.28[\times 2]\left(\mathrm{C}-4^{\mathrm{A}, \mathrm{B}, \mathrm{D}, \mathrm{E}}\right), 92.46$, 92.84, 94.98, 95.99, 96.35, 96.79, $96.89(\mathrm{C}-1), 125.92$ (virtual t, $\left.\right|^{3} J_{\mathrm{C}, \mathrm{P}}+{ }^{5} J_{\mathrm{C}, \mathrm{P}} \mid=9.8 \mathrm{~Hz}, m-\mathrm{C}$ ), 126.57 (virtual t, $\left.\right|^{3} J_{\mathrm{C}, \mathrm{P}}+{ }^{5} J_{\mathrm{C}, \mathrm{P}} \mid=9.5 \mathrm{~Hz}, m-\mathrm{C}$ ), $128.11,128.33(p-\mathrm{C}), 130.97$ (virtual t, $\left.\right|^{2} J_{\mathrm{C}, \mathrm{P}}+$ $\left.{ }^{4} J_{\mathrm{C}, \mathrm{P}}{ }^{\prime} \mid=12.7 \mathrm{~Hz}, o-\mathrm{C}\right), 131.37$ (virtual t, $\left.\left.\right|^{2} J_{\mathrm{C}, \mathrm{P}}+{ }^{4} J_{\mathrm{C}, \mathrm{P}}{ }^{\prime} \mid=12.7 \mathrm{~Hz}, o-\mathrm{C}\right), 132.87$ [ $\left.\times 2\right]$ (virtual t, $\left.\right|^{2} J_{\mathrm{C}, \mathrm{P}}+{ }^{4} J_{\mathrm{C}, \mathrm{P}} \mid=51.0 \mathrm{~Hz}$, ipso-C) ppm; ${ }^{31} \mathrm{P}\left\{{ }^{1} \mathrm{H}\right\} \mathrm{NMR}\left(121.5 \mathrm{MHz}, \mathrm{CDCl}_{3}, 25^{\circ} \mathrm{C}\right): \delta=15.5(\mathrm{br}$ s) ppm; ${ }^{31} \mathrm{P}\left\{{ }^{1} \mathrm{H}\right\}$ NMR $\left(121.5 \mathrm{MHz}, \mathrm{CD}_{2} \mathrm{Cl}_{2},-80^{\circ} \mathrm{C}\right)=21.7$ and $11.4\left(2 \mathrm{~d}, \mathrm{AB}\right.$ system, ${ }^{2} J_{\mathrm{P} 1, \mathrm{P} 2}$ $=564 \mathrm{~Hz}), 17.7$ and $5.6\left(2 \mathrm{~d}, \mathrm{AB}\right.$ system, $\left.{ }^{2} J_{\mathrm{P} 1, \mathrm{P} 2}=549 \mathrm{~Hz}\right) \mathrm{ppm}$; elemental analysis (\%) calcd for $\mathrm{C}_{71} \mathrm{H}_{110} \mathrm{Cl}_{2} \mathrm{O}_{31} \mathrm{P}_{2} \mathrm{Pd}$ (1698.89): C 50.20, $\mathrm{H}$ 6.53, found: $\mathrm{C} 50.12, \mathrm{H}$ 6.74; MS (ESI-TOF): $m / z(\%): 1737.46(19)[M+\mathrm{K}]^{+}, 1721.47(76)[M+\mathrm{Na}]^{+}, 1661.51(5)[M-\mathrm{Cl}]^{+}$.

trans-P,P'-Dichlorido- $\left\{6^{\mathrm{A}}, 6^{\mathrm{B}}, 6^{\mathrm{D}}, 6^{\mathrm{E}}\right.$-tetradeoxy- $6^{\mathrm{A}}, 6^{\mathrm{B}}: 6^{\mathrm{D}}, 6^{\mathrm{E}}$-bis $[(R)$-phenylphosphinidene $]-$ $2^{\mathrm{A}}, 2^{\mathrm{B}}, 2^{\mathrm{C}}, 2^{\mathrm{D}}, 2^{\mathrm{E}}, 2^{\mathrm{F}}, 2^{\mathrm{G}}, 3^{\mathrm{A}}, 3^{\mathrm{B}}, 3^{\mathrm{C}}, 3^{\mathrm{D}}, 3^{\mathrm{E}}, 3^{\mathrm{F}}, 3^{\mathrm{G}}, 6^{\mathrm{C}}, 6^{\mathrm{F}}, 6^{\mathrm{G}}$-heptadeca- $O$-methyl- $\beta$-cyclodextrin $\}$ pla tinum(II) (10)

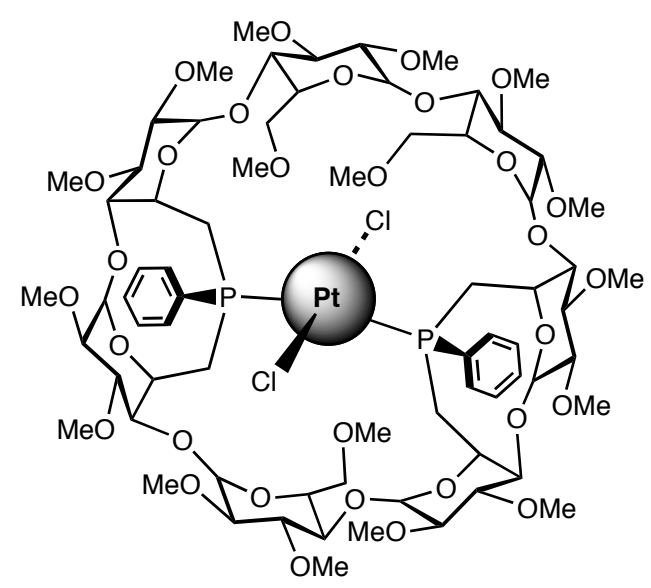

A solution of $\left[\mathrm{PtCl}_{2}(\mathrm{PhCN})_{2}\right](0.025 \mathrm{~g}, 0.053 \mathrm{mmol})$ in $\mathrm{CH}_{2} \mathrm{Cl}_{2}(5 \mathrm{~mL})$ was added dropwise to a solution of diphosphine $6(0.080 \mathrm{~g}, 0.053 \mathrm{mmol})$ in $\mathrm{CH}_{2} \mathrm{Cl}_{2}(10 \mathrm{~mL})$ within $30 \mathrm{~min}$ at room 
temperature. After $30 \mathrm{~min}$, the reaction mixture was evaporated to dryness affording analytically pure $10(0.089 \mathrm{~g}, 99 \%)$ as a pale yellow solid. $R_{\mathrm{f}}\left(\mathrm{SiO}_{2}, \mathrm{CH}_{2} \mathrm{Cl}_{2} / \mathrm{MeOH}, 92: 8, v / v\right)$ $=0.40$; m.p. dec.; ${ }^{1} \mathrm{H}$ NMR $\left(300.1 \mathrm{MHz}, \mathrm{CDCl}_{3}, 25^{\circ} \mathrm{C}\right): \delta($ assignment by COSY $)=2.12-2.21$ $\left(\mathrm{m}, 1 \mathrm{H}, \mathrm{H}-6 \mathrm{a}^{\mathrm{A}}\right.$ or D), $2.34\left(\mathrm{dt}, 1 \mathrm{H},{ }^{3} J_{\mathrm{H}-6 \mathrm{a}, \mathrm{P}}=15.5 \mathrm{~Hz},{ }^{2} J_{\mathrm{H}-6 \mathrm{a}, \mathrm{H}-6 \mathrm{~b}}=7.7 \mathrm{~Hz},{ }^{3} J_{\mathrm{H}-6 \mathrm{a}, \mathrm{H}-5}=7.7 \mathrm{~Hz}\right.$, $\left.\mathrm{H}-6 \mathrm{a}^{\mathrm{D} \text { or A }}\right), 2.68-2.76\left(2 \mathrm{H}, \mathrm{H}-6 \mathrm{a}^{\mathrm{B}, \mathrm{E}}\right), 2.82\left(\mathrm{dd}, 1 \mathrm{H},{ }^{3} J_{\mathrm{H}-2, \mathrm{H}-3}=9.2 \mathrm{~Hz},{ }^{3} J_{\mathrm{H}-2, \mathrm{H}-1}=3.1 \mathrm{~Hz}, \mathrm{H}-2\right)$, 3.05-3.75 (22 H, H-2, H-3, H-4 $\left.{ }^{\mathrm{A}, \mathrm{B}, \mathrm{D}, \mathrm{E}}, \mathrm{H}-6 \mathrm{a}^{\mathrm{C}, \mathrm{F}, \mathrm{G}}, \mathrm{H}-6 \mathrm{~b}^{\mathrm{B}, \mathrm{E}}\right), 3.27$ (s, $\left.3 \mathrm{H}, \mathrm{OMe}\right), 3.30$ (s, $3 \mathrm{H}$, OMe), 3.32 (s, 3 H, OMe), 3.38 (s, 3 H, OMe), 3.45 (s, 3 H, OMe) 3.48 (s, 3 H, OMe), 3.50 (s, $3 \mathrm{H}, \mathrm{OMe}), 3.53$ (s, $3 \mathrm{H}, \mathrm{OMe}), 3.57$ (s, $3 \mathrm{H}, \mathrm{OMe}), 3.59$ (s, $6 \mathrm{H}, \mathrm{OMe}), 3.62$ (s, $6 \mathrm{H}$, OMe), 3.63 (s, 3 H, OMe), 3.64 (s, 3 H, OMe), 3.72 (s, 3 H, OMe), 3.84 (s, 3 H, OMe), 3.86$3.95\left(6 \mathrm{H}, \mathrm{H}-4^{\mathrm{C}, \mathrm{F}, \mathrm{G}}, \mathrm{H}-6 \mathrm{~b}^{\mathrm{A}, \mathrm{D} \text { and } \mathrm{C} \text { or F or G }}\right), 4.02-4.11\left(4 \mathrm{H}, \mathrm{H}-5^{\mathrm{F}, \mathrm{G} \text { or C,F or C,G }}, \mathrm{H}-6 \mathrm{~b}^{\mathrm{F}, \mathrm{G} \text { or C,F or C,G }}\right)$, $4.25\left(\mathrm{~d}, 1 \mathrm{H},{ }^{3} J_{\mathrm{H}-5, \mathrm{H}-6 \mathrm{a}}=9.7 \mathrm{~Hz}, \mathrm{H}-5^{\mathrm{C} \text { or F or G }}\right), 4.33-4.39\left(3 \mathrm{H}, \mathrm{H}-5^{\mathrm{A} \text { or D and B,E }}\right), 4.55(\mathrm{~m}, 1 \mathrm{H}$, $\left.\mathrm{H}-5^{\text {D or A }}\right), 4.80\left(\mathrm{~d}, 1 \mathrm{H},{ }^{3} J_{\mathrm{H}-1, \mathrm{H}-2}=3.3 \mathrm{~Hz}, \mathrm{H}-1\right), 4.91\left(\mathrm{~d}, 1 \mathrm{H},{ }^{3} J_{\mathrm{H}-1, \mathrm{H}-2}=3.4 \mathrm{~Hz}, \mathrm{H}-1\right), 5.03(\mathrm{~d}$, $\left.1 \mathrm{H},{ }^{3} J_{\mathrm{H}-1, \mathrm{H}-2}=4.0 \mathrm{~Hz}, \mathrm{H}-1\right), 5.06\left(\mathrm{~d}, 2 \mathrm{H},{ }^{3} J_{\mathrm{H}-1, \mathrm{H}-2}=4.3 \mathrm{~Hz}, \mathrm{H}-1\right), 5.27\left(\mathrm{~d}, 1 \mathrm{H},{ }^{3} J_{\mathrm{H}-1, \mathrm{H}-2}=4.1\right.$ $\mathrm{Hz}, \mathrm{H}-1), 5.54\left(\mathrm{~d}, 1 \mathrm{H},{ }^{3} J_{\mathrm{H}-1, \mathrm{H}-2}=4.7 \mathrm{~Hz}, \mathrm{H}-1\right), 7.39-7.42(6 \mathrm{H}, m-\mathrm{H}, p-\mathrm{H}), 8.06-8.10(4 \mathrm{H}, o-$ H) ppm; ${ }^{13} \mathrm{C}\left\{{ }^{1} \mathrm{H}\right\} \mathrm{NMR}\left(75.5 \mathrm{MHz}, \mathrm{CDCl}_{3}, 25^{\circ} \mathrm{C}\right): \delta$ (assignment by HMQC) $=22.80,26.50$ $\left(\mathrm{C}-6^{\mathrm{A}, \mathrm{D} \text { or B,E }}\right), 32.00,33.00$ (C-6 ${ }^{\mathrm{B}, \mathrm{E}}$ or A,D $), 56.73,57.74,57.91,58.15,58.79,58.84,58.93$, $59.02,59.78,60.42,60.62,60.96,61.27,61.40,61.54,61.72,61.89(\mathrm{OMe}), 63.54,64.29$ (C$\left.5^{\mathrm{B}, \mathrm{E} \text { or A,D }}\right), 67.97,69.10\left(\mathrm{C}-5^{\mathrm{A}, \mathrm{D} \text { or B,E}}\right), 70.37,70.45,70.69\left(\mathrm{C}-5^{\mathrm{C}, \mathrm{F}, \mathrm{G}}\right), 71.02,71.89,72.05(\mathrm{C}-$ $\left.6^{\mathrm{C}, \mathrm{F}, \mathrm{G}}\right), 77.90[\times 2], 78.07\left(\mathrm{C}-4^{\mathrm{C}, \mathrm{F}, \mathrm{G}}\right), 79.02[\times 2], 79.70[\times 2], 80.54,81.13[\times 2], 81.37,81.61$, $82.11,82.43,82.95,83.05,83.44(\mathrm{C}-2, \mathrm{C}-3), 84.50[\times 2]\left(\mathrm{C}-4^{\mathrm{A}, \mathrm{D}}\right.$ or B,E $), 89.39[\times 2]\left(\mathrm{C}-4^{\mathrm{B}, \mathrm{E}}\right.$ or ${ }^{\mathrm{A}, \mathrm{D}}$ ), 94.64, 94.75, 96.97, 98.14, 98.26, 98.75, 99.25 (C-1), 127.95 (virtual t, $\left.\right|^{3} J_{\mathrm{C}, \mathrm{P}}+{ }^{5} J_{\mathrm{C}, \mathrm{P}}{ }^{\prime} \mid=$ $10.0 \mathrm{~Hz}, m-\mathrm{C}$ ), 128.52 (virtual t, $\left.\left|{ }^{3} J_{\mathrm{C}, \mathrm{P}}+{ }^{5} J_{\mathrm{C}, \mathrm{P}}{ }^{\prime}\right|=10.0 \mathrm{~Hz}, m-\mathrm{C}\right), 130.28[\times 2](p-\mathrm{C}), 133.25$ (virtual t, $\left.\right|^{2} J_{\mathrm{C}, \mathrm{P}}+{ }^{4} J_{\mathrm{C}, \mathrm{P}}{ }^{\prime} \mid=12.3 \mathrm{~Hz}, o-\mathrm{C}$ ), 133.69 (virtual t, $\left.\right|^{2} J_{\mathrm{C}, \mathrm{P}}+{ }^{4} J_{\mathrm{C}, \mathrm{P}} \mid=12.3 \mathrm{~Hz}, o-\mathrm{C}$ ), $135.57[\times 2]$ (virtual t, $\left|{ }^{1} J_{\mathrm{C}, \mathrm{P}}+{ }^{3} J_{\mathrm{C}, \mathrm{P}}\right|=52.0 \mathrm{~Hz}$, ipso-C) ppm; ${ }^{31} \mathrm{P}\left\{{ }^{1} \mathrm{H}\right\} \mathrm{NMR}(121.5 \mathrm{MHz}$, $\mathrm{CDCl}_{3}, 25^{\circ} \mathrm{C}$ ): $\delta=8.9$ (br s with br Pt satellites, $\left.{ }^{1} J_{\mathrm{P}, \mathrm{Pt}} \approx 2500 \mathrm{~Hz}\right) \mathrm{ppm} ;{ }^{31} \mathrm{P}\left\{{ }^{1} \mathrm{H}\right\} \mathrm{NMR}(121.5$ $\left.\mathrm{MHz}, \mathrm{CD}_{2} \mathrm{Cl}_{2},-80^{\circ} \mathrm{C}\right)=12.2$ and $1.0\left(2 \mathrm{~d}, \mathrm{AB}\right.$ system, $\left.{ }^{2} J_{\mathrm{P} 1, \mathrm{P} 2}=476 \mathrm{~Hz}\right), 17.8$ and $5.0(2 \mathrm{~d}$, $\mathrm{AB}$ system, $\left.{ }^{2} J_{\mathrm{P} 1, \mathrm{P} 2}=492 \mathrm{~Hz}\right) \mathrm{ppm}\left({ }^{1} J_{\mathrm{Pt}, \mathrm{P}}\right.$ poorly resolved $) ;{ }^{31} \mathrm{P}\left\{{ }^{1} \mathrm{H}\right\} \mathrm{NMR}(202.5 \mathrm{MHz}$, $\left.\mathrm{C}_{2} \mathrm{D}_{2} \mathrm{Cl}_{4}, 80^{\circ} \mathrm{C}\right)=7.35$ and $7.0\left(2 \mathrm{~d}\right.$ with Pt satellites, $\mathrm{ABX}$ system, ${ }^{1} J_{\mathrm{Pt}, \mathrm{P} 1}=2507 \mathrm{~Hz},{ }^{1} J_{\mathrm{Pt}, \mathrm{P} 2}=$ $\left.2481 \mathrm{~Hz},{ }^{2} J_{\mathrm{P} 1, \mathrm{P} 2}=496 \mathrm{~Hz}\right) \mathrm{ppm}$. elemental analysis $(\%)$ calcd for $\mathrm{C}_{71} \mathrm{H}_{110} \mathrm{Cl}_{2} \mathrm{O}_{31} \mathrm{P}_{2} \mathrm{Pt}$ (1785.55): C 47.71, H 6.20, found: C 47.91, H 6.31; MS (ESI-TOF): $m / z$ (\%): 1792.45 (100) $[M+\mathrm{Li}]^{+}$. 
trans- $P, P^{\prime}$-chlorido-carbonyl- $\left\{6^{\mathrm{A}}, 6^{\mathrm{B}}, 6^{\mathrm{D}}, 6^{\mathrm{E}}\right.$-tetradeoxy- $6^{\mathrm{A}}, 6^{\mathrm{B}}: 6^{\mathrm{D}}, 6^{\mathrm{E}}-\mathrm{bis}[(R)-$

phenylphosphinidene $]-2^{\mathrm{A}}, 2^{\mathrm{B}}, 2^{\mathrm{C}}, 2^{\mathrm{D}}, 2^{\mathrm{E}}, 2^{\mathrm{F}}, 2^{\mathrm{G}}, 3^{\mathrm{A}}, 3^{\mathrm{B}}, 3^{\mathrm{C}}, 3^{\mathrm{D}}, 3^{\mathrm{E}}, 3^{\mathrm{F}}, 3^{\mathrm{G}}, 6^{\mathrm{C}}, 6^{\mathrm{F}}, 6^{\mathrm{G}}$-heptadeca- $O$ -

methyl- $\beta$-cyclodextrin\}rhodium(I) (11)

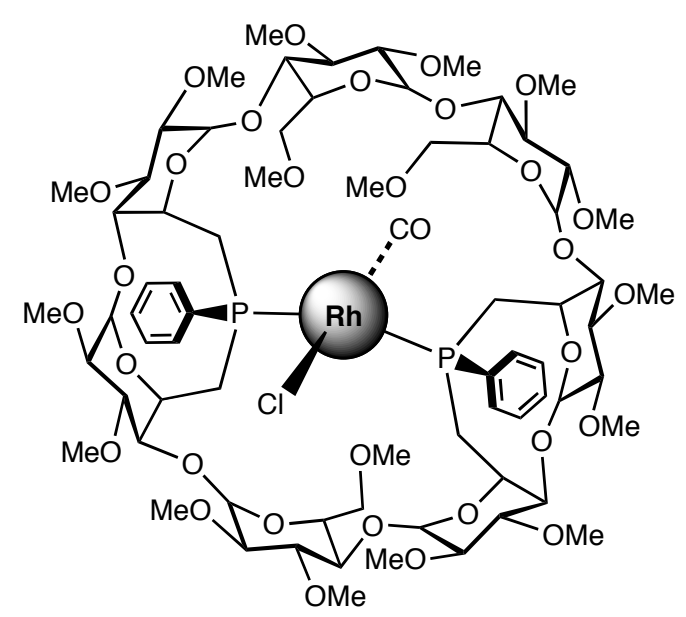

A solution of $\left[\mathrm{RhCl}(\mathrm{CO})_{2}\right]_{2}(0.010 \mathrm{~g}, 0.026 \mathrm{mmol})$ in $\mathrm{CH}_{2} \mathrm{Cl}_{2}(5 \mathrm{~mL})$ was added dropwise to a solution of diphosphine $6(0.080 \mathrm{~g}, 0.053 \mathrm{mmol})$ in $\mathrm{CH}_{2} \mathrm{Cl}_{2}(10 \mathrm{~mL})$ within $30 \mathrm{~min}$ at room temperature. After stirring for $30 \mathrm{~min}$, the reaction mixture was evaporated to dryness affording analytically pure $10(0.089 \mathrm{~g}, 99 \%)$ as an orange-yellow solid. $R_{\mathrm{f}}\left(\mathrm{SiO}_{2}\right.$, $\left.\mathrm{CH}_{2} \mathrm{Cl}_{2} / \mathrm{MeOH}, 92: 8, v / v\right)=0.40$; m.p. dec.; IR (KBr) $v / \mathrm{cm}^{-1}: 1970 ;{ }^{1} \mathrm{H}$ NMR $(300.1 \mathrm{MHz}$, $\left.\mathrm{CDCl}_{3}, 25^{\circ} \mathrm{C}\right): \delta$ (assignment by COSY $)=2.25-2.38\left(2 \mathrm{H}, \mathrm{H}-6 \mathrm{a}^{\mathrm{A}, \mathrm{D} \text { or B,E}}\right), 2.55-2.65(2 \mathrm{H}, \mathrm{H}-$ $\left.6 \mathrm{a}^{\mathrm{B}, \mathrm{E} \text { or A,D }}\right), 2.78\left(\mathrm{~d}, 1 \mathrm{H},{ }^{3} J_{\mathrm{H}-2, \mathrm{H}-3}=9.0 \mathrm{~Hz}, \mathrm{H}-2\right), 3.05-4.05\left(32 \mathrm{H}, \mathrm{H}-2, \mathrm{H}-3, \mathrm{H}-4, \mathrm{H}-5^{\mathrm{C}, \mathrm{F} \text { or C,G }}\right.$

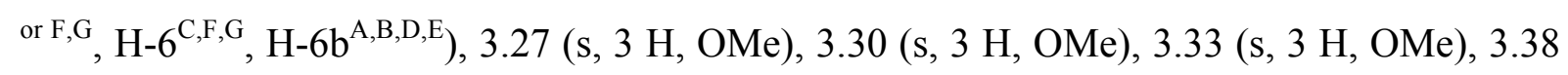
(s, $3 \mathrm{H}, \mathrm{OMe}), 3.46(\mathrm{~s}, 3 \mathrm{H}, \mathrm{OMe}) 3.48(\mathrm{~s}, 3 \mathrm{H}, \mathrm{OMe}), 3.50(\mathrm{~s}, 3 \mathrm{H}, \mathrm{OMe}), 3.53$ (s, $3 \mathrm{H}$, OMe), 3.57 (s, 3 H, OMe), 3.59 (s, 6 H, OMe), 3.60 (s, 3 H, OMe), 3.62 (s, 3 H, OMe), 3.64 $(\mathrm{s}, 6 \mathrm{H}, \mathrm{OMe}), 3.71(\mathrm{~s}, 3 \mathrm{H}, \mathrm{OMe}), 3.81(\mathrm{~s}, 3 \mathrm{H}, \mathrm{OMe}), 4.20\left(\mathrm{~d}, 1 \mathrm{H},{ }^{3} J_{\mathrm{H}-5, \mathrm{H}-6 \mathrm{a}}=9.4 \mathrm{~Hz}, \mathrm{H}-5^{\mathrm{G}}\right.$ or F or C $), 4.38-4.53\left(4 \mathrm{H}, \mathrm{H}-5^{\mathrm{A}, \mathrm{B}, \mathrm{D}, \mathrm{E}}\right), 4.83\left(\mathrm{~d}, 1 \mathrm{H},{ }^{3} J_{\mathrm{H}-1, \mathrm{H}-2}=3.4 \mathrm{~Hz}, \mathrm{H}-1\right), 4.91\left(\mathrm{~d}, 1 \mathrm{H},{ }^{3} J_{\mathrm{H}-1, \mathrm{H}-}\right.$ $\left.{ }_{2}=3.1 \mathrm{~Hz}, \mathrm{H}-1\right), 5.06\left(\mathrm{~d}, 1 \mathrm{H},{ }^{3} J_{\mathrm{H}-1, \mathrm{H}-2}=4.1 \mathrm{~Hz}, \mathrm{H}-1\right), 5.07\left(\mathrm{~d}, 1 \mathrm{H},{ }^{3} J_{\mathrm{H}-1, \mathrm{H}-2}=4.5 \mathrm{~Hz}, \mathrm{H}-1\right)$, $5.11\left(\mathrm{~d}, 1 \mathrm{H},{ }^{3} J_{\mathrm{H}-1, \mathrm{H}-2}=2.9 \mathrm{~Hz}, \mathrm{H}-1\right), 5.31\left(\mathrm{~d}, 1 \mathrm{H},{ }^{3} J_{\mathrm{H}-1, \mathrm{H}-2}=4.1 \mathrm{~Hz}, \mathrm{H}-1\right), 5.48\left(\mathrm{~d}, 1 \mathrm{H},{ }^{3} J_{\mathrm{H}-}\right.$ 1,H-2 $=4.5 \mathrm{~Hz}, \mathrm{H}-1), 7.35-7.39(6 \mathrm{H}, m-\mathrm{H}, p-\mathrm{H}), 7.99-8.07(4 \mathrm{H}, o-\mathrm{H}) \mathrm{ppm} ;{ }^{13} \mathrm{C}\left\{{ }^{1} \mathrm{H}\right\} \mathrm{NMR}$ $\left(75.5 \mathrm{MHz}, \mathrm{CDCl}_{3}, 25^{\circ} \mathrm{C}\right): \delta$ (assignment by HMQC) $=28.40[\times 2]\left(\right.$ virtual $\mathrm{t},\left|{ }^{1} J_{\mathrm{C}, \mathrm{P}}+{ }^{3} J_{\mathrm{C}, \mathrm{P}}{ }^{\prime}\right|=$ $\left.9.9 \mathrm{~Hz}, \mathrm{C}-6^{\mathrm{A}, \mathrm{D} \text { or B,E }}\right), 30.19[\times 2]$ (virtual t, $\left|{ }^{1} J_{\mathrm{C}, \mathrm{P}}+{ }^{3} J_{\mathrm{C}, \mathrm{P}}\right|=11.4 \mathrm{~Hz}, \mathrm{C}-6^{\mathrm{B}, \mathrm{E} \text { or A,D }}$ ), 56.75, 57.89 [×2], 58.10, 58.72, 58.82, 58.92, 50.00, 59.44, 60.31, 60.57, 60.93 [×2], 61.06, 61.18, 61.69, $61.72(\mathrm{OMe}), 64.10,64.79\left(\mathrm{C}-5^{\mathrm{A}, \mathrm{D} \text { or B,E }}\right), 68.60,69.63\left(\mathrm{C}-5^{\mathrm{B}, \mathrm{E}}\right.$ or A,D$), 69.92,70.41,70.90(\mathrm{C}-$ $\left.5^{\mathrm{C}, \mathrm{F}, \mathrm{G}}\right), 71.08,71.69,72.30\left(\mathrm{C}-6^{\mathrm{C}, \mathrm{F}, \mathrm{G}}\right), 77.21[\times 2], 77.64\left(\mathrm{C}-4^{\mathrm{C}, \mathrm{F}, \mathrm{G}}\right), 79.57,79.82,80.34,80.97$, 
$81.24,81.36[\times 3], 82.38,82.53,83.08[\times 2], 83.50,84.42,(\mathrm{C}-2, \mathrm{C}-3), 84.95[\times 2]\left(\mathrm{C}-4^{\mathrm{A}, \mathrm{D}}\right.$ or $\left.{ }^{\mathrm{B}, \mathrm{E}}\right), 89.51[\times 2]\left(\mathrm{C}-4^{\mathrm{B}, \mathrm{E} \text { or A,D}}\right), 94.36,94.57,97.01,98.02,98.34,98.47,99.08(\mathrm{C}-1), 127.68$ (virtual t, $\left|{ }^{3} J_{\mathrm{C}, \mathrm{P}}+{ }^{5} J_{\mathrm{C}, \mathrm{P}}\right|=4.5 \mathrm{~Hz}, m-\mathrm{C}$ ), 128.39 (virtual t, $\left|{ }^{3} J_{\mathrm{C}, \mathrm{P}}+{ }^{5} J_{\mathrm{C}, \mathrm{P}}\right|=4.5 \mathrm{~Hz}, m-\mathrm{C}$ ), 129.72, $129.82(p-\mathrm{C}), 133.21$ (virtual t, $\left.\right|^{2} J_{\mathrm{C}, \mathrm{P}}+{ }^{4} J_{\mathrm{C}, \mathrm{P}} \mid=6.3 \mathrm{~Hz}, o-\mathrm{C}$ ), 133.58 (virtual t, $\left.\right|^{2} J_{\mathrm{C}, \mathrm{P}}+$ $\left.{ }^{4} J_{\mathrm{C}, \mathrm{P}} \mid=5.8 \mathrm{~Hz}, o-\mathrm{C}\right), 137.97[\times 2]$ (virtual t, $\left|{ }^{1} J_{\mathrm{C}, \mathrm{P}}+{ }^{3} J_{\mathrm{C}, \mathrm{P}}\right|=22.5 \mathrm{~Hz}$, ipso-C) ppm; ${ }^{31} \mathrm{P}\left\{{ }^{1} \mathrm{H}\right\}$ $\operatorname{NMR}\left(121.5 \mathrm{MHz}, \mathrm{CDCl}_{3}, 25^{\circ} \mathrm{C}\right): \delta=18.7$ (br d, $\left.{ }^{1} J_{\mathrm{P}, \mathrm{Rh}} \approx 120 \mathrm{~Hz}\right) \mathrm{ppm} ;{ }^{31} \mathrm{P}\left\{{ }^{1} \mathrm{H}\right\} \mathrm{NMR}(162.0$ $\left.\mathrm{MHz}, \mathrm{CD}_{2} \mathrm{Cl}_{2},-80^{\circ} \mathrm{C}\right)=28.0$ and $9.4\left(2 \mathrm{dd}, \mathrm{ABX}\right.$ system, ${ }^{2} J_{\mathrm{P} 1, \mathrm{P} 2}=354 \mathrm{~Hz},{ }^{1} J_{\mathrm{P}, \mathrm{Rh}}=118$ and 118), 16.4 and 9.4 (2 dd, $\mathrm{ABX}$ system, ${ }^{2} J_{\mathrm{P} 1, \mathrm{P} 2}=354 \mathrm{~Hz},{ }^{1} J_{\mathrm{P}, \mathrm{Rh}}=118$ and 118) ppm; elemental analysis (\%) calcd for $\mathrm{C}_{72} \mathrm{H}_{110} \mathrm{ClO}_{32} \mathrm{P}_{2} \mathrm{Rh}$ (1687.93): C 51.23, H 6.57, found: C 51.15, H 6.44; MS (ESI-TOF): $m / z(\%): 1725.48(23)[M+\mathrm{K}]^{+}, 1709.51(58)[M+\mathrm{Na}]^{+}, 1652.51(16)[M-$ $\mathrm{Cl}]^{+}$.

\section{Reaction of $\left[\mathrm{PdCl}_{2}(\mathrm{PhCN})_{2}\right]$ with 9 and formation of 12.}

A solution of $\left[\mathrm{PdCl}_{2}(\mathrm{PhCN})_{2}\right](0.040 \mathrm{~g}, 0.106 \mathrm{mmol})$ in $\mathrm{CH}_{2} \mathrm{Cl}_{2}(5 \mathrm{~mL})$ was added to a solution of diphosphine $6(0.080 \mathrm{~g}, 0.053 \mathrm{mmol})$ in $\mathrm{CH}_{2} \mathrm{Cl}_{2}(10 \mathrm{~mL})$ at room temperature. After $30 \mathrm{~min}$, the reaction mixture was concentrated to $c a .2 \mathrm{~mL}$ and pentane $(50 \mathrm{~mL})$ was added. The suspension was then filtered over celite. Evaporation of pentane afforded a yellow powder $(0.099 \mathrm{~g}, 99 \%)$ of formula $\mathrm{C}_{71} \mathrm{H}_{110} \mathrm{Cl}_{4} \mathrm{O}_{31} \mathrm{P}_{2} \mathrm{Pd}_{2}$ (12'), the exact structure of which is not known. $R_{\mathrm{f}}\left(\mathrm{SiO}_{2}, \mathrm{CH}_{2} \mathrm{Cl}_{2} / \mathrm{MeOH}, 92: 8, v / v\right)=0.35$; m.p. dec.; ${ }^{1} \mathrm{H}$ NMR $(300.1 \mathrm{MHz}$, $\left.\mathrm{CDCl}_{3}, 25^{\circ} \mathrm{C}\right): \delta($ assignment by COSY $)=1.71\left(\mathrm{dt}, 1 \mathrm{H},{ }^{2} J_{\mathrm{H}-6 \mathrm{a}, \mathrm{P}}=13.9 \mathrm{~Hz},{ }^{2} J_{\mathrm{H}-6 \mathrm{a}, \mathrm{H}-6 \mathrm{~b}}={ }^{3} J_{\mathrm{H}-6 \mathrm{a}, \mathrm{H} 5}\right.$ $=12.1 \mathrm{~Hz}, \mathrm{H}-6 \mathrm{a}^{\mathrm{A}}$ or D) $2.16\left(\mathrm{dt}, 1 \mathrm{H},{ }^{2} J_{\mathrm{H}-6 \mathrm{a}, \mathrm{P}}=13.1 \mathrm{~Hz},{ }^{2} J_{\mathrm{H}-6 \mathrm{a}, \mathrm{H}-6 \mathrm{~b}}={ }^{3} J_{\mathrm{H}-6 \mathrm{a}, \mathrm{H}-5}=12.4 \mathrm{~Hz}, \mathrm{H}-6 \mathrm{a}^{\mathrm{D}}\right.$ or $\left.{ }^{\mathrm{A}}\right), 2.78-2.83\left(2 \mathrm{H}, \mathrm{H}-6^{\mathrm{B}}\right.$ or E$), 2.98-3.03\left(2 \mathrm{H}, \mathrm{H}-6^{\mathrm{E} \text { or B}}\right), 3.07-3.34\left(12 \mathrm{H}, \mathrm{H}-2, \mathrm{H}-4^{\mathrm{A}, \mathrm{B}, \mathrm{D}, \mathrm{E}}, \mathrm{H}-\right.$ $\left.6 \mathrm{~b}^{\mathrm{A} \text { or } \mathrm{D}}\right), 3.48-3.88\left(11 \mathrm{H}, \mathrm{H}-3, \mathrm{H}-4^{\mathrm{C}, \mathrm{F}, \mathrm{G}}, \mathrm{H}-6 \mathrm{a}^{\mathrm{C} \text { or F or G}}\right), 3.43(\mathrm{~s}, 3 \mathrm{H}, \mathrm{OMe}), 3.47(\mathrm{~s}, 3 \mathrm{H}$, OMe), 3.51 (s, $6 \mathrm{H}, \mathrm{OMe}$ ), 3.51 (s, $3 \mathrm{H}, \mathrm{OMe}$ ), 3.52 (s, $3 \mathrm{H}, \mathrm{OMe}), 3.53$ (s, $3 \mathrm{H}, \mathrm{OMe}), 3.54$ (s, $3 \mathrm{H}, \mathrm{OMe}$ ), 3.58 (s, $3 \mathrm{H}, \mathrm{OMe}), 3.62$ (s, $3 \mathrm{H}, \mathrm{OMe}), 3.65$ (s, $3 \mathrm{H}, \mathrm{OMe}), 3.66$ (s, $3 \mathrm{H}$, OMe), 3.67 (s, 3 H, OMe), 3.68 (s, 3 H, OMe), 3.70 (s, 3 H, OMe), 3.74 (s, 3 H, OMe), 3.77 $(\mathrm{s}, 3 \mathrm{H}, \mathrm{OMe}), 4.05\left(\mathrm{dd}, 1 \mathrm{H},{ }^{2} J_{\mathrm{H}-6 \mathrm{~b}, \mathrm{H}-6 \mathrm{a}}=14.7 \mathrm{~Hz},{ }^{3} J_{\mathrm{H}-6 \mathrm{~b}, \mathrm{H}-5}=9.2 \mathrm{~Hz}, \mathrm{H}-6 \mathrm{~b}^{\mathrm{D}}\right.$ or A $) 4.11-4.17(2$ $\left.\mathrm{H}, \mathrm{H}-5^{\mathrm{C} \text { or F or G }}, \mathrm{H}-6 \mathrm{~b}^{\mathrm{C} \text { or F or G}}\right), 4.27\left(\mathrm{~d}, 1 \mathrm{H},{ }^{2} J_{\mathrm{H}-6 \mathrm{~b}, \mathrm{H}-6 \mathrm{a}}=10.9 \mathrm{~Hz}, \mathrm{H}-6 \mathrm{a}^{\mathrm{F} \text { or G or C}}\right), 4.37(\mathrm{~d}, 1 \mathrm{H}$, $\left.{ }^{3} J_{\mathrm{H}-5, \mathrm{H}-6 \mathrm{a}}=10.0 \mathrm{~Hz}, \mathrm{H}-5^{\mathrm{F} \text { or G or C}}\right), 4.47-4.58\left(3 \mathrm{H}, \mathrm{H}-5^{\mathrm{A} \text { or D }}, \mathrm{H}-5^{\mathrm{G}}\right.$ or C or F, $\left.\mathrm{H}-6 \mathrm{a}^{\mathrm{G} \text { or C or F }}\right), 4.75$ $\left(\mathrm{dd}, 1 \mathrm{H},{ }^{2} J_{\mathrm{H}-6 \mathrm{~b}, \mathrm{H}-6 \mathrm{a}}=10.7 \mathrm{~Hz},{ }^{3} J_{\mathrm{H}-6 \mathrm{~b}, \mathrm{H}-5}=1.9 \mathrm{~Hz}, \mathrm{H}-6 \mathrm{~b}^{\mathrm{F} \text { or G or C}}\right), 4.82\left(\mathrm{~m}, 1 \mathrm{H}, \mathrm{H}-5^{\mathrm{D} \text { or A }}\right), 4.90$ $\left(\mathrm{d}, 1 \mathrm{H},{ }^{3} J_{\mathrm{H}-1, \mathrm{H}-2}=4.7 \mathrm{~Hz}, \mathrm{H}-1\right), 4.93\left(\mathrm{dd}, 1 \mathrm{H},{ }^{2} J_{\mathrm{H}-6 \mathrm{~b}, \mathrm{H}-6 \mathrm{a}}=11.1 \mathrm{~Hz},{ }^{3} J_{\mathrm{H}-6 \mathrm{~b}, \mathrm{H}-5}=1.8 \mathrm{~Hz}, \mathrm{H}-6 \mathrm{~b}^{\mathrm{G}}\right.$ or $\mathrm{C}$ or $\mathrm{F}), 4.99\left(\mathrm{~d}, 1 \mathrm{H},{ }^{3} J_{\mathrm{H}-1, \mathrm{H}-2}=4.4 \mathrm{~Hz}, \mathrm{H}-1\right), 5.00\left(\mathrm{~d}, 1 \mathrm{H},{ }^{3} J_{\mathrm{H}-1, \mathrm{H}-2}=4.1 \mathrm{~Hz}, \mathrm{H}-1\right), 5.06(\mathrm{~m}, 1$ $\left.\mathrm{H}, \mathrm{H}-5^{\text {B or E }}\right), 5.12\left(\mathrm{~d}, 1 \mathrm{H},{ }^{3} J_{\mathrm{H}-1, \mathrm{H}-2}=4.4 \mathrm{~Hz}, \mathrm{H}-1\right), 5.14\left(\mathrm{~d}, 1 \mathrm{H},{ }^{3} J_{\mathrm{H}-1, \mathrm{H}-2}=2.9 \mathrm{~Hz}, \mathrm{H}-1\right), 5.16$ $\left(\mathrm{d}, 1 \mathrm{H},{ }^{3} J_{\mathrm{H}-1, \mathrm{H}-2}=3.6 \mathrm{~Hz}, \mathrm{H}-1\right), 5.21\left(\mathrm{~m}, 1 \mathrm{H}, \mathrm{H}-5^{\mathrm{E} \text { or B }}\right), 5.28\left(\mathrm{~d}, 1 \mathrm{H},{ }^{3} J_{\mathrm{H}-1, \mathrm{H}-2}=2.9 \mathrm{~Hz}, \mathrm{H}-1\right)$, 
7.37-7.46 (6 H, $m-\mathrm{H}, p-\mathrm{H}), 7.52-7.57(2 \mathrm{H}, o-\mathrm{H}), 7.64-7.67(2 \mathrm{H}, o-\mathrm{H}) \mathrm{ppm} ;{ }^{13} \mathrm{C}\left\{{ }^{1} \mathrm{H}\right\} \mathrm{NMR}$ $\left(75.5 \mathrm{MHz}, \mathrm{CDCl}_{3}, 25^{\circ} \mathrm{C}\right): \delta$ (assignment by $\left.\mathrm{HMQC}\right)=31.54\left(\mathrm{~d}, J_{\mathrm{C}, \mathrm{P}}=31.8 \mathrm{~Hz}, \mathrm{C}-6^{\mathrm{D} \text { or A}}\right)$, $31.12\left(\mathrm{~d}, J_{\mathrm{C}, \mathrm{P}}=32.5 \mathrm{~Hz}, \mathrm{C}-6^{\mathrm{A} \text { or D }}\right), 35.48\left(\mathrm{~d}, J_{\mathrm{C}, \mathrm{P}}=29.7 \mathrm{~Hz}, \mathrm{C}-6^{\mathrm{E} \text { or B }}\right), 38.08\left(\mathrm{~d}, J_{\mathrm{C}, \mathrm{P}}=23.1 \mathrm{~Hz}\right.$, C-6 $\left.{ }^{\mathrm{B} \text { or } \mathrm{E}}\right), 57.40,57.83,58.01,58.62,58.66,58.72,59.32,59.36,59.68,59.84,61.31[\times 2]$, $61.55,61.71,61.82,61.86,61.92(\mathrm{OMe}), 64.35\left(\mathrm{C}-5^{\mathrm{D} \text { or A }}\right), 64.59\left(\mathrm{C}-5^{\mathrm{A} \text { or D }}\right), 68.67\left(\mathrm{C}-5^{\mathrm{E}}\right.$ or B $)$, $69.49\left(\mathrm{C}-5^{\mathrm{B} \text { or E}}\right), 71.16,71.45,71.70\left(\mathrm{C}-5^{\mathrm{C}, \mathrm{F}, \mathrm{G}}\right), 71.63,72.78[\times 2]\left(\mathrm{C}-6^{\mathrm{C}, \mathrm{F}, \mathrm{G}}\right), 79.98,80.26$, 80.49 [×2], 80.92, 81.31, 81.59 [×2], $82.04[\times 2], 82.18$ [×2], 82.73, 82.83, 82.96, 83.15 [×2] $\left(\mathrm{C}-2, \mathrm{C}-3, \mathrm{C}-4^{\mathrm{C}, \mathrm{F}, \mathrm{G}}\right), 84.11\left(\mathrm{~d},{ }^{3} J_{\mathrm{C}, \mathrm{P}}=11.4 \mathrm{~Hz}\right), 87.60\left(\mathrm{~d},{ }^{3} J_{\mathrm{C}, \mathrm{P}}=11.4 \mathrm{~Hz}\right), 89.64,89.83(\mathrm{C}-$ $\left.4^{\mathrm{A}, \mathrm{B}, \mathrm{D}, \mathrm{E}}\right), 97.55,98.14,98.26,98.85,99.79,100.72,102.07(\mathrm{C}-1), 128.55\left(\mathrm{~d},{ }^{3} J_{\mathrm{C}, \mathrm{P}}=11.6 \mathrm{~Hz}\right.$, $m-\mathrm{C}), 128.70\left(\mathrm{~d},{ }^{3} J_{\mathrm{C}, \mathrm{P}}=11.6 \mathrm{~Hz}, m-\mathrm{C}\right), 131.02,131.10(p-\mathrm{C}), 131.19,131.29(o-\mathrm{C}), 130.7-$ 131.94 [×2] (ipso-C) pmm; ${ }^{31} \mathrm{P}\left\{{ }^{1} \mathrm{H}\right\} \mathrm{NMR}\left(121.5 \mathrm{MHz} \mathrm{CDCl}_{3}, 25^{\circ} \mathrm{C}\right): \delta=22.4$ (br s), 27.3 (d, ${ }^{4} J_{\mathrm{P}, \mathrm{P}},=4.4 \mathrm{~Hz}$ ) ppm; elemental analysis (\%) calcd for $\mathrm{C}_{71} \mathrm{H}_{110} \mathrm{Cl}_{4} \mathrm{O}_{31} \mathrm{P}_{2} \mathrm{Pd}_{2}(1876.21)$ : C 45.45, H 5.91, found: C 45.39, H 6.09; MS (ESI-TOF): $m / z(\%): 1899.32(75)[M+\mathrm{Na}]^{+}, 1839.40$ (25) $[M-\mathrm{Cl}]^{+}$. Recristallisation of 12 ' in $\mathrm{CH}_{2} \mathrm{Cl}_{2}$-pentane (undistilled) gave 12 (vide infra, $\mathrm{X}$ ray analysis).

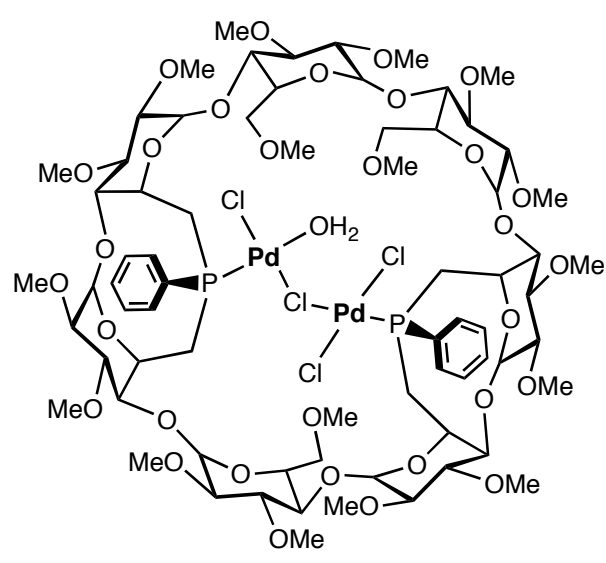

12

Dichlorido- $\left\{6^{\mathrm{A}}, 6^{\mathrm{B}}, 6^{\mathrm{D}}, 6^{\mathrm{E}}\right.$-tetradeoxy-6 ${ }^{\mathrm{A}}, 6^{\mathrm{B}}[(R)$-phenylphosphinidene $]-6^{\mathrm{D}}, 6^{\mathrm{E}}-[(S)$-phenyl oxophosphinidene $]-2^{\mathrm{A}}, 2^{\mathrm{B}}, 2^{\mathrm{C}}, 2^{\mathrm{D}}, 2^{\mathrm{E}}, 2^{\mathrm{F}}, 2^{\mathrm{G}}, 3^{\mathrm{A}}, 3^{\mathrm{B}}, 3^{\mathrm{C}}, 3^{\mathrm{D}}, 3^{\mathrm{E}}, 3^{\mathrm{F}}, 3^{\mathrm{G}}, 6^{\mathrm{C}}, 6^{\mathrm{F}}, 6^{\mathrm{G}}$-heptadeca- $O$ methyl- $\beta$-cyclodextrin $\}$ palladium(II) (13) and dichloro- $\left\{6^{\mathrm{A}}, 6^{\mathrm{B}}, 6^{\mathrm{D}}, 6^{\mathrm{E}}\right.$-tetradeoxy- $6^{\mathrm{A}}, 6^{\mathrm{B}}[(S)$ phenyloxophosphinidene $]-6^{\mathrm{D}}, 6^{\mathrm{E}}-[(R)$-phenylphosphinidene $]-2^{\mathrm{A}}, 2^{\mathrm{B}}, 2^{\mathrm{C}}, 2^{\mathrm{D}}, 2^{\mathrm{E}}, 2^{\mathrm{F}}, 2^{\mathrm{G}}, 3^{\mathrm{A}}, 3^{\mathrm{B}}, 3^{\mathrm{C}}$, $3^{\mathrm{D}}, 3^{\mathrm{E}}, 3^{\mathrm{F}}, 3^{\mathrm{G}}, 6^{\mathrm{C}}, 6^{\mathrm{F}}, 6^{\mathrm{G}}$-heptadeca- $\boldsymbol{O}$-methyl- $\beta$-cyclodextrin $\}$ palladium(II) (14) 


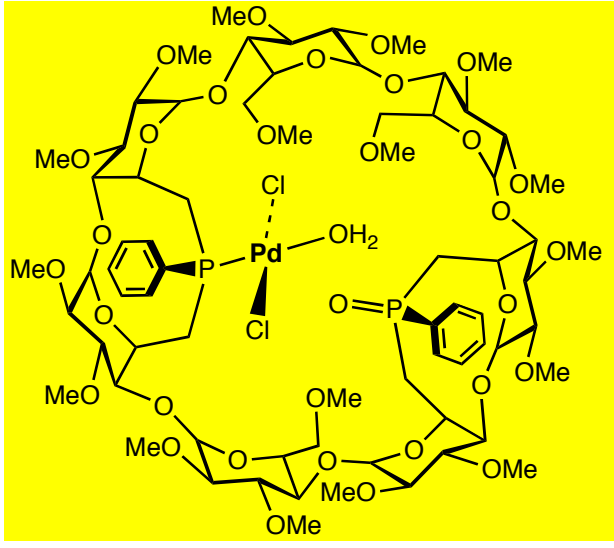

13

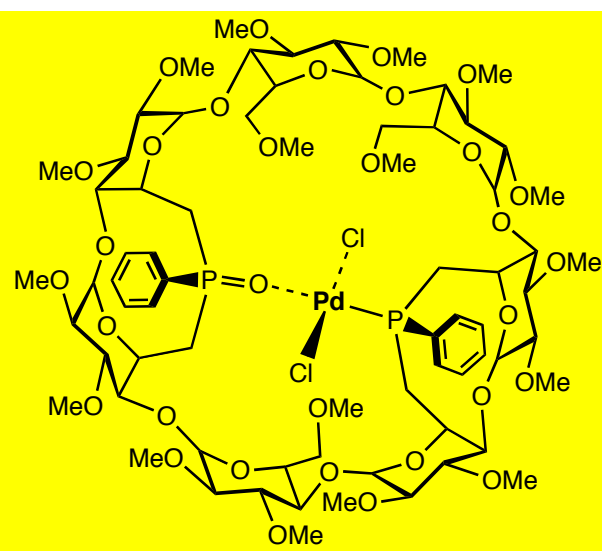

14

Air was bubbled through a solution of complex $9(0.080 \mathrm{~g}, 0.047 \mathrm{mmol})$ in undistilled $\mathrm{MeOH}$ for $30 \mathrm{~min}$. After refluxing the solution for $12 \mathrm{~h}$, the solvent was removed in vacuo and the crude product was purified by column chromatography* $\left(\mathrm{SiO}_{2}, \mathrm{CH}_{2} \mathrm{Cl}_{2} / \mathrm{MeOH}, 97: 3, v / v\right)$ to give compound $13(0.036 \mathrm{~g}, 45 \%)$ as a pale yellow solid. $R_{\mathrm{f}}\left(\mathrm{SiO}_{2}, \mathrm{CH}_{2} \mathrm{Cl}_{2} / \mathrm{MeOH}, 92: 8, v / v\right)$ $=0.35$; m.p. dec.; ${ }^{1} \mathrm{H}$ NMR $\left(600.1 \mathrm{MHz}, \mathrm{CDCl}_{3}, 25^{\circ} \mathrm{C}\right): \delta($ assignment by COSY $)=1.85(\mathrm{~m}, 1$ $\left.\mathrm{H}, \mathrm{H}-6 \mathrm{a}^{\mathrm{D} \text { or E}}\right), 1.91-1.96\left(2 \mathrm{H}, \mathrm{H}-6 \mathrm{a}^{\mathrm{A}}\right.$ or B and $\mathrm{E}$ or $\left.\mathrm{D}\right), 2.80\left(\mathrm{dd}, 1 \mathrm{H},{ }^{1} J_{\mathrm{H}-6 \mathrm{a}, \mathrm{P}}=18.6 \mathrm{~Hz},{ }^{2} J_{\mathrm{H}-6 \mathrm{a}, \mathrm{H}-6 \mathrm{~b}}=\right.$ $15.8 \mathrm{~Hz}, \mathrm{H}-6 \mathrm{~b}^{\mathrm{A}}$ or B$), 2.99-3.04\left(2 \mathrm{H}, \mathrm{H}-6^{\mathrm{B}}\right.$ or A $), 3.07-3.80\left(31 \mathrm{H}, \mathrm{H}-2, \mathrm{H}-3, \mathrm{H}-4, \mathrm{H}-5^{\mathrm{D}, \mathrm{E}}, \mathrm{H}-\right.$ $\left.6^{\mathrm{C}, \mathrm{F}, \mathrm{G}}, \mathrm{H}-6 \mathrm{~b}^{\mathrm{D}, \mathrm{E}}\right), 3.15$ (s, $\left.6 \mathrm{H}, \mathrm{OMe}\right), 3.35$ (s, $\left.3 \mathrm{H}, \mathrm{OMe}\right), 3,46$ (s, $\left.3 \mathrm{H}, \mathrm{OMe}\right), 3.50$ (s, $3 \mathrm{H}$, OMe), 3.51 (s, $3 \mathrm{H}, \mathrm{OMe}$ ), 3.52 (s, $3 \mathrm{H}, \mathrm{OMe}$ ), 3.53 (s, $3 \mathrm{H}, \mathrm{OMe}), 3.54$ (s, $6 \mathrm{H}, \mathrm{OMe}), 3.59$ (s, $3 \mathrm{H}, \mathrm{OMe}), 3.64$ (s, $6 \mathrm{H}, \mathrm{OMe}), 3.67$ (s, $3 \mathrm{H}, \mathrm{OMe}), 3.69$ (s, $3 \mathrm{H}, \mathrm{OMe}), 3.74$ (s, $3 \mathrm{H}$, OMe), 3.76 (s, $3 \mathrm{H}, \mathrm{OMe}), 3.96-4.02\left(2 \mathrm{H}, \mathrm{H}-5^{\mathrm{C}, \mathrm{F}}\right.$ or C,G or F,G $), 4.25\left(\mathrm{~d}, 1 \mathrm{H},{ }^{3} J_{\mathrm{H}-5, \mathrm{H}-6}=10.4 \mathrm{~Hz}\right.$, $\left.\mathrm{H}-5^{\mathrm{G} \text { or F or C}}\right), 4.54\left(\mathrm{~m}, 1 \mathrm{H}, \mathrm{H}-5^{\mathrm{A} \text { or B }}\right), 4.82\left(\mathrm{~m}, 1 \mathrm{H}, \mathrm{H}-5^{\mathrm{B} \text { or A }}\right), 4.94\left(\mathrm{~d}, 2 \mathrm{H},{ }^{3} J_{\mathrm{H}-1, \mathrm{H}-2}=2.7 \mathrm{~Hz}\right.$, $\mathrm{H}-1), 4.99\left(\mathrm{~d}, 1 \mathrm{H},{ }^{3} J_{\mathrm{H}-1, \mathrm{H}-2}=3.9 \mathrm{~Hz}, \mathrm{H}-1\right), 5.00\left(\mathrm{~d}, 1 \mathrm{H},{ }^{3} J_{\mathrm{H}-1, \mathrm{H}-2}=3.9 \mathrm{~Hz}, \mathrm{H}-1\right), 5.03$ (d, $1 \mathrm{H}$, $\left.{ }^{3} J_{\mathrm{H}-1, \mathrm{H}-2}=3.9 \mathrm{~Hz}, \mathrm{H}-1\right), 5.06(\mathrm{~m}, 1 \mathrm{H}, \mathrm{H}-1), 5.09(\mathrm{~m}, 1 \mathrm{H}, \mathrm{H}-1), 7.35\left(\mathrm{td}, 2 \mathrm{H},{ }^{3} J_{m-\mathrm{H}, o-\mathrm{H}}={ }^{3} J_{m-}\right.$ $\mathrm{H}, p-\mathrm{H}=7.9 \mathrm{~Hz},{ }^{4} J_{m-\mathrm{H}, \mathrm{P}}=0.5 \mathrm{~Hz}, m-\mathrm{H}$ of $\left.(\mathrm{O}) \mathrm{PPh}\right), 7.41\left(\mathrm{t}, 1 \mathrm{H},{ }^{3} J_{p-\mathrm{H}, m-\mathrm{H}}=7.9 \mathrm{~Hz}, p-\mathrm{H}\right.$ of (O)PPh), 7.57-7.59 (3 H, $m-\mathrm{H}$ and $p-\mathrm{H}$ of $\mathrm{PdPPh}), 7.86\left(\mathrm{dd}, 2 \mathrm{H},{ }^{3} J_{o-\mathrm{H}, \mathrm{P}}=11.8 \mathrm{~Hz},{ }^{3} J_{o-\mathrm{H}, m-\mathrm{H}}=\right.$ $7.9 \mathrm{~Hz}, o-\mathrm{H}$ of $(\mathrm{O}) \mathrm{PPh}), 8.22(\mathrm{~m}, 2 \mathrm{H}, o-\mathrm{H}$ of $\mathrm{PdPPh}) \mathrm{ppm} ;{ }^{13} \mathrm{C}\left\{{ }^{1} \mathrm{H}\right\} \mathrm{NMR}(75.5 \mathrm{MHz}$, $\left.\mathrm{CDCl}_{3}, 25^{\circ} \mathrm{C}\right): \delta$ (assignment by $\left.\mathrm{HMQC}\right)=57.31,57.87,58.07,58.54,58.78,58.83,58.91$, $59.12,59.32,59.55,61.26,61.38,61.72[\times 2], 61.83[\times 2], 62.09(\mathrm{OMe}), 62.98\left(\mathrm{C}-5^{\mathrm{A}}\right.$ or B $)$, $66.38\left(\mathrm{C}-5^{\mathrm{B} \text { or A}}\right), 70.46[\times 2]\left(\mathrm{C}-6^{\mathrm{C}, \mathrm{F} \text { or C,G or F,G }}\right), 70.66[\times 2]\left(\mathrm{C}-5^{\mathrm{C}, \mathrm{F} \text { or C,G or F,G }}\right), 71.50\left(\mathrm{C}-6^{\mathrm{G} \text { or F }}\right.$ or $\mathrm{C}), 71.68\left(\mathrm{C}-5^{\mathrm{G}}\right.$ or $\mathrm{F}$ or $\left.\mathrm{C}\right), 73.45[\times 2]\left(\mathrm{C}-5^{\mathrm{D}, \mathrm{E}}\right), 78.39[\times 2], 80.79,81.03[\times 2], 81.13,81.24$, $81.71,81.87,81.96,82.94$ [×2], 83.04 [×2], 83.24, 83.58, 83.68 (C-2, C-3, C-4 $\left.{ }^{\mathrm{C}, \mathrm{F}, \mathrm{G}}\right), 86.40$, $86.93\left(\mathrm{C}-4^{\mathrm{D}, \mathrm{E}}\right), 89.36[\times 2]\left(\mathrm{C}-4^{\mathrm{A}, \mathrm{B}}\right), 95.33,97.94,98.86,100.61,100.65,100.90,101.23(\mathrm{C}-$ 1), $128.16\left(\mathrm{~d},{ }^{3} J_{\mathrm{C}, \mathrm{P}}=11.7 \mathrm{~Hz}, m-\mathrm{C}\right.$ of $\left.(\mathrm{O}) \mathrm{PPh}\right), 128.87\left(\mathrm{~d},{ }^{3} J_{\mathrm{C}, \mathrm{P}}=11.4 \mathrm{~Hz}, m-\mathrm{C}\right.$ of PdPPh$)$, $130.25\left(\mathrm{~d},{ }^{2} J_{\mathrm{C}, \mathrm{P}}=10.2 \mathrm{~Hz}, o-\mathrm{C}\right.$ of $\left.\mathrm{PdPPh}\right), 130.74(p-\mathrm{C}$ of $(\mathrm{O}) \mathrm{PPh}), 132.11\left(\mathrm{~d},{ }^{2} J_{\mathrm{C}, \mathrm{P}}=9.7 \mathrm{~Hz}\right.$, 
$o-\mathrm{C}$ of $(\mathrm{O}) \mathrm{PPh}), 132.48(p-\mathrm{C}$ of $\mathrm{PdPPh}) \mathrm{ppm}\left(\mathrm{C}-6^{\mathrm{A}, \mathrm{B}, \mathrm{D}, \mathrm{E}}\right.$ and both ipso-C's could not be

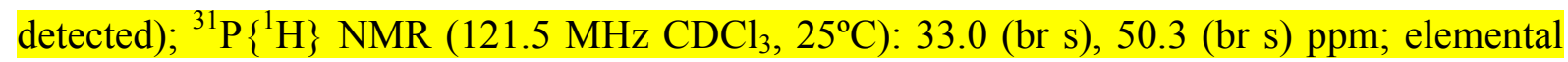
analysis (\%) calcd for $\mathrm{C}_{71} \mathrm{H}_{112} \mathrm{Cl}_{2} \mathrm{O}_{33} \mathrm{P}_{2} \mathrm{Pd}$ (1732.90): $\mathrm{C} 49.21, \mathrm{H}$ 6.51, found: $\mathrm{C} 49.39, \mathrm{H}$ 5.69; MS (ESI-TOF): $m / z$ (\%): 1737.52 (50) $\left[M+\mathrm{Li}^{+}, 1735.48(50)\left[M-\mathrm{H}_{2} \mathrm{O}+\mathrm{Na}\right]^{+}\right.$.* During the purification, complex $14(0.034 \mathrm{~g}, 43 \%)$ was also isolated. $R_{\mathrm{f}}\left(\mathrm{SiO}_{2}\right.$, $\left.\mathrm{CH}_{2} \mathrm{Cl}_{2} / \mathrm{MeOH}, 92: 8, v / v\right)=0.40$; m.p. dec.; ${ }^{1} \mathrm{H}$ NMR $\left(600.1 \mathrm{MHz}, \mathrm{CDCl}_{3}, 25^{\circ} \mathrm{C}\right): \delta$ $($ assignment by COSY $)=2.08\left(\mathrm{~m}, 1 \mathrm{H}, \mathrm{H}-6 \mathrm{a}^{\mathrm{A}}\right.$ or $\left.\mathrm{B}\right), 2.17\left(\mathrm{~m}, 1 \mathrm{H}, \mathrm{H}-6 \mathrm{a}^{\mathrm{D} \text { or E}}\right), 2.38(\mathrm{~m}, 1 \mathrm{H}, \mathrm{H}-$ $\left.6 \mathrm{a}^{\mathrm{E} \text { or D }}\right), 2.52\left(\mathrm{td}, 1 \mathrm{H},{ }^{2} J_{\mathrm{H}-6 \mathrm{a}, \mathrm{H}-6 \mathrm{~b}}={ }^{2} J_{\mathrm{H}-6 \mathrm{a}, \mathrm{P}}=14.4 \mathrm{~Hz},{ }^{2} J_{\mathrm{H}-6 \mathrm{a}, \mathrm{H}-5}=5.7 \mathrm{~Hz}, \mathrm{H}-6 \mathrm{a}^{\mathrm{B}}\right.$ or A $), 2.75(\mathrm{t}, 1$ $\mathrm{H},{ }^{2} J_{\mathrm{H}-6 \mathrm{~b}, \mathrm{H}-6 \mathrm{a}}={ }^{2} J_{\mathrm{H}-6 \mathrm{~b}, \mathrm{P}}=15.2 \mathrm{~Hz}, \mathrm{H}-6 \mathrm{~b}^{\mathrm{B}}$ or A $), 2.80\left(\mathrm{dd}, 1 \mathrm{H},{ }^{2} J_{\mathrm{H}-6 \mathrm{~b}, \mathrm{H}-6 \mathrm{ba}}=16.4 \mathrm{~Hz},{ }^{3} J_{\mathrm{H}-6 \mathrm{~b}, \mathrm{H}-5}=\right.$ $7.3 \mathrm{~Hz}, \mathrm{H}-6 \mathrm{~b}^{\mathrm{D}}$ or $\left.\mathrm{E}\right), 3.01-3.82\left(25 \mathrm{H}, \mathrm{H}-2, \mathrm{H}-3, \mathrm{H}-4, \mathrm{H}-6 \mathrm{a}^{\mathrm{C}, \mathrm{F}, \mathrm{G}}, \mathrm{H}-6 \mathrm{~b}^{\mathrm{E}}\right), 3.24$ (s, $\left.3 \mathrm{H}, \mathrm{OMe}\right)$, 3.31 (s, $3 \mathrm{H}, \mathrm{OMe}), 3,32$ (s, $3 \mathrm{H}, \mathrm{OMe}$ ), 3.46 (s, $3 \mathrm{H}, \mathrm{OMe}), 3.47$ (s, $3 \mathrm{H}, \mathrm{OMe}), 3.48$ (s, $3 \mathrm{H}$, OMe), 3.49 (s, $6 \mathrm{H}, \mathrm{OMe}), 3.59$ (s, $6 \mathrm{H}, \mathrm{OMe}), 3.60$ (s, $3 \mathrm{H}, \mathrm{OMe}), 3.62$ (s, $6 \mathrm{H}, \mathrm{OMe}), 3.64$ (s, $3 \mathrm{H}, \mathrm{OMe}), 3.65$ (s, $3 \mathrm{H}, \mathrm{OMe}), 3.74$ (s, $3 \mathrm{H}, \mathrm{OMe}), 3.85$ (s, $3 \mathrm{H}, \mathrm{OMe}), 3.95\left(\mathrm{dd}, 1 \mathrm{H},{ }^{2} J_{\mathrm{H}-}\right.$ $\left.6 \mathrm{~b}, \mathrm{H}-6 \mathrm{a}=11.5 \mathrm{~Hz},{ }^{3} J_{\mathrm{H}-6 \mathrm{~b}, \mathrm{H}-5}=1.5 \mathrm{~Hz}, \mathrm{H}-6 \mathrm{~b}^{\mathrm{C} \text { or F or G }}\right), 4.05\left(\mathrm{~d}, 1 \mathrm{H},{ }^{2} J_{\mathrm{H}-6 \mathrm{~b}, \mathrm{H}-6 \mathrm{a}}=11.7 \mathrm{~Hz},{ }^{3} J_{\mathrm{H}-6 \mathrm{~b}, \mathrm{H}-5}\right.$ $\left.=1.9 \mathrm{~Hz}, \mathrm{H}-6 \mathrm{~b}^{\mathrm{F} \text { or G or C}}\right), 4.11\left(\mathrm{~d}, 1 \mathrm{H},{ }^{2} J_{\mathrm{H}-6 \mathrm{~b}, \mathrm{H}-6 \mathrm{a}}=9.8 \mathrm{~Hz}, \mathrm{H}-6 \mathrm{~b}^{\mathrm{A} \text { or B }}\right), 4.14\left(\mathrm{~m}, 1 \mathrm{H}, \mathrm{H}-5^{\mathrm{C} \text { or F or }}\right.$ G), $4.15\left(\mathrm{dd}, 1 \mathrm{H},{ }^{2} J_{\mathrm{H}-6 \mathrm{~b}, \mathrm{H}-6 \mathrm{a}}=10.9 \mathrm{~Hz},{ }^{3} J_{\mathrm{H}-6 \mathrm{~b}, \mathrm{H}-5}=2.4 \mathrm{~Hz}, \mathrm{H}-6 \mathrm{~b}^{\mathrm{G} \text { or C or F}}\right), 4.48\left(\mathrm{~m}, 1 \mathrm{H}, \mathrm{H}-5^{\mathrm{A} \text { or }}\right.$ $\left.{ }^{\mathrm{B}}\right), 4.35\left(\mathrm{~m}, 1 \mathrm{H}, \mathrm{H}-5^{\mathrm{G} \text { or } \mathrm{C} \text { or } \mathrm{F}}\right), 4.42\left(2 \mathrm{H}, \mathrm{H}-5^{\mathrm{B}}\right.$ or A and $\mathrm{F}$ or $\mathrm{G}$ or $\left.\mathrm{C}\right), 4.49\left(2 \mathrm{H}, \mathrm{H}-5^{\mathrm{D}, \mathrm{E}}\right), 4.84(\mathrm{~d}, 1$ $\left.\mathrm{H},{ }^{3} J_{\mathrm{H}-1, \mathrm{H}-2}=3.3 \mathrm{~Hz}, \mathrm{H}-1\right), 4.97\left(\mathrm{~d}, 1 \mathrm{H},{ }^{3} J_{\mathrm{H}-1, \mathrm{H}-2}=4.3 \mathrm{~Hz}, \mathrm{H}-1\right), 4.99\left(\mathrm{~d}, 1 \mathrm{H},{ }^{3} J_{\mathrm{H}-1, \mathrm{H}-2}=4.3\right.$ $\mathrm{Hz}, \mathrm{H}-1), 5.06$ (d, $\left.1 \mathrm{H},{ }^{3} J_{\mathrm{H}-1, \mathrm{H}-2}=3.8 \mathrm{~Hz}, \mathrm{H}-1\right), 5.07$ (d, $\left.1 \mathrm{H},{ }^{3} J_{\mathrm{H}-1, \mathrm{H}-2}=4.5 \mathrm{~Hz}, \mathrm{H}-1\right), 5.23$ (d, 1 $\left.\mathrm{H},{ }^{3} J_{\mathrm{H}-1, \mathrm{H}-2}=4.2 \mathrm{~Hz}, \mathrm{H}-1\right), 5.26\left(\mathrm{~d}, 1 \mathrm{H},{ }^{3} J_{\mathrm{H}-1, \mathrm{H}-2}=4.3 \mathrm{~Hz}, \mathrm{H}-1\right), 7.42-7.45\left(\mathrm{td}, 2 \mathrm{H},{ }^{3} J_{m-\mathrm{H}, \mathrm{o}-\mathrm{H}}=\right.$ ${ }^{3} J_{m-\mathrm{H}, p-\mathrm{H}}=7.9 \mathrm{~Hz},{ }^{4} J_{m-\mathrm{H}, \mathrm{P}}=2.0 \mathrm{~Hz}, m-\mathrm{H}$ of $\left.(\mathrm{O}) \mathrm{PPh}\right), 7.47(\mathrm{~m}, 1 \mathrm{H}, p-\mathrm{H}$ of $(\mathrm{O}) \mathrm{PPh}), 7.58$ (td, 2 $\mathrm{H},{ }^{3} J_{m-\mathrm{H}, o-\mathrm{H}}={ }^{3} J_{m-\mathrm{H}, p-\mathrm{H}}=7.6 \mathrm{~Hz},{ }^{4} J_{m-\mathrm{H}, \mathrm{P}}=2.6 \mathrm{~Hz}, m-\mathrm{H}$ of PdPPh), $7.62(\mathrm{~m}, 1 \mathrm{H}, p-\mathrm{H}$ of PdPPh), 8.05-8.10 (4 H, o-H) ppm; ${ }^{13} \mathrm{C}\left\{{ }^{1} \mathrm{H}\right\}$ NMR $\left(150.9 \mathrm{MHz}, \mathrm{CDCl}_{3}, 25^{\circ} \mathrm{C}\right): \delta$ (assignment by $\mathrm{HMQC})=31.77\left(\mathrm{~d},{ }^{1} J_{\mathrm{C}, \mathrm{P}}=26.8 \mathrm{~Hz}, \mathrm{C}-6^{\mathrm{A}}\right.$ or B $), 32.12\left(\left(\mathrm{~d},{ }^{1} J_{\mathrm{C}, \mathrm{P}}=59.4 \mathrm{~Hz}, \mathrm{C}-6^{\mathrm{E}}\right.\right.$ or D$), 37.61(\mathrm{~d}$, ${ }^{1} J_{\mathrm{C}, \mathrm{P}}=31.9 \mathrm{~Hz}, \mathrm{C}-6^{\mathrm{B}}$ or A $), 42.14\left(\mathrm{~d},{ }^{1} J_{\mathrm{C}, \mathrm{P}}=72.4 \mathrm{~Hz}, \mathrm{C}-6^{\mathrm{D}}\right.$ or $\left.\mathrm{E}\right), 59.84,56.96,57.84,58.10$, $58.79,58.85[\times 2], 58.92,60.29,60.39,60.61,60.93,61.70,61.73[\times 2], 61.94[\times 2](\mathrm{OMe})$, $61.98\left(\mathrm{C}-5^{\mathrm{E} \text { or D}}\right), 63.21\left(\mathrm{~d},{ }^{2} J_{\mathrm{C}, \mathrm{P}}=4.0 \mathrm{~Hz}, \mathrm{C}-5^{\mathrm{A} \text { or B }}\right), 66.06\left(\mathrm{C}-5^{\mathrm{D} \text { or E}}\right), 68.27\left(\mathrm{~d},{ }^{2} J_{\mathrm{C}, \mathrm{P}}=3.5 \mathrm{~Hz}\right.$, $\left.\mathrm{C}-5^{\mathrm{B} \text { or } \mathrm{A}}\right), 70.42[\times 2], 70.09\left(\mathrm{C}-5^{\mathrm{C}, \mathrm{F}, \mathrm{G}}\right), 71.20,71.57,72.22\left(\mathrm{C}-6^{\mathrm{C}, \mathrm{F}, \mathrm{G}}\right), 77.29,80.27,80.36$, $80.47,80.54,81.00,81.14,81.17,81.68,81.75,81.84,81.90,82.06,82.08,82.43,82.82$, $82.93,83.45,83.81,84.26\left(\mathrm{C}-2, \mathrm{C}-3, \mathrm{C}-4^{\mathrm{A}}\right.$ or B and C,D,E,F,G$), 89.31\left(\mathrm{~d},{ }^{3} J_{\mathrm{C}, \mathrm{P}}=12.8 \mathrm{~Hz}, \mathrm{C}-4^{\mathrm{B}}\right.$ or A $)$, 94.20, 97.42, 98.11, 98.90, 99.49, 99.64, 99.78 (C-1), 128.66 (d, $\left.{ }^{3} J_{\mathrm{C}, \mathrm{P}}=5.9 \mathrm{~Hz}, m-\mathrm{C}\right), 128.73$ $\left(\mathrm{d},{ }^{3} J_{\mathrm{C}, \mathrm{P}}=6.2 \mathrm{~Hz}, m-\mathrm{C}\right), 130.11\left(\mathrm{~d},{ }^{2} J_{\mathrm{C}, \mathrm{P}}=9.1 \mathrm{~Hz}, o-\mathrm{C}\right.$ of $\left.(\mathrm{O}) \mathrm{PPh}\right), 131.03(p-\mathrm{C}$ of $(\mathrm{O}) \mathrm{PPh})$, $132.13(p-\mathrm{C}$ of $\mathrm{PdPPh}), 132.74\left(\mathrm{~d},{ }^{2} J_{\mathrm{C}, \mathrm{P}}=9.1 \mathrm{~Hz}, o-\mathrm{C}\right.$ of $\left.\mathrm{PdPPh}\right), 133.72\left(\mathrm{~d},{ }^{1} J_{\mathrm{C}, \mathrm{P}}=6.7 \mathrm{~Hz}\right.$, ipso-C of (O)PPh), $134.24\left(\mathrm{~d},{ }^{1} J_{\mathrm{C}, \mathrm{P}}=6.7 \mathrm{~Hz}\right.$, ipso-C of PdPPh) ppm; ${ }^{31} \mathrm{P}\left\{{ }^{1} \mathrm{H}\right\} \mathrm{NMR}(121.5$ 
$\left.\mathrm{MHz} \mathrm{CDCl}_{3}, 25^{\circ} \mathrm{C}\right): 33.8\left(\mathrm{~d},{ }^{2} J_{\mathrm{P}, \mathrm{P}}=4.4 \mathrm{~Hz}\right), 54.5\left(\mathrm{~d},{ }^{2} J_{\mathrm{P}, \mathrm{P}}=4.4 \mathrm{~Hz}\right) \mathrm{ppm}$; elemental analysis (\%) calcd for $\mathrm{C}_{71} \mathrm{H}_{110} \mathrm{Cl}_{2} \mathrm{O}_{32} \mathrm{P}_{2} \mathrm{Pd}$ (1714.89): $\mathrm{C} 49.73, \mathrm{H} \mathrm{6.47}$, found: $\mathrm{C} 49.60, \mathrm{H}$ 6.55; MS (ESI-TOF): $m / z(\%): 1753.47(30)[M+\mathrm{K}]^{+}, 1737.49(70)[M+\mathrm{Na}]^{+}$.

Crystal structure of 7•0.5 $\mathbf{C H}_{\mathbf{2}} \mathbf{C l}_{\mathbf{2}} \bullet \mathbf{0 . 5} \mathbf{C}_{\mathbf{5}} \mathbf{H}_{\mathbf{1 2}}: M \mathrm{r}=2163.56$, orthorhombic, $P 2_{1} 2_{1} 2_{1}, a=$ $15.1479(3), b=15.6542(3), c=36.0599(9) \AA, V=8550.8(3) \AA^{3}, Z=4, D_{\mathrm{X}}=1.268 \mathrm{mg} \mathrm{m}^{-3}$, $\lambda(\mathrm{MoK} \alpha)=0.71073 \AA, \mu=0.163 \mathrm{~mm}^{-1}, F(000)=3488, T=150(1) \mathrm{K}$. Data were collected on an Oxford Diffraction Xcalibur Saphir 3 diffractometer (graphite MoK $\alpha$ radiation, $\lambda=$ $0.71073 \AA$ ). The structure was solved with SIR-9 ${ }^{[3]}$ which revealed the non hydrogen atoms of the molecule. After anisotropic refinement, many hydrogen atoms were found with a Fourier Difference. The whole structure was refined with SHELXL97 ${ }^{[4]}$ by the full-matrix least-square techniques (use of $F$ square magnitude; $\mathrm{x}, \mathrm{y}, \mathrm{z}, \beta_{\mathrm{ij}}$ for $\mathrm{Pd}, \mathrm{P}, \mathrm{Cl}, \mathrm{C}$ and $\mathrm{O}$ atoms, $\mathrm{x}, \mathrm{y}, \mathrm{z}$ in riding mode for $\mathrm{H}$ atoms; 988 variables and 18635 observations with $I>2.0 \sigma(I)$; calc $w=1 /\left[\sigma^{2}\left(F \mathrm{o}^{2}\right)+(0.0985 P)^{2}+0.0000 P\right]$ where $P=\left(F \mathrm{o}^{2}+2 F \mathrm{c}^{2}\right) / 3 . R=0.071, R_{\mathrm{W}}=$ 0.190 and $S_{\mathrm{W}}=0.791, \Delta \rho<0.645 \mathrm{e}^{-3}$. The alerts level A in the checkcif are mainly due to disordered $\mathrm{MeO}$ groups and solvents molecules. CCDC 756653 contains the supplementary crystallographic data for this paper. Crystallographic data can be obtained free of charge at www.ccdc.cam.ac.uk/conts/retrieving.html [or from the Cambridge Crystallographic Data Centre, 12 Union Road, Cambridge CB 11EZ, UK; ax: (internat.) 44-1223/336-033; E-mail: deposit@ccdc.cam.ac.uk].

Crystal structure of $\mathbf{1 2} \cdot \mathbf{0 . 5} \mathbf{C}_{\mathbf{5}} \mathbf{H}_{\mathbf{1 2}}: M r=1930.22$, monoclinic, $P 2_{1} 2_{1} 2_{1}, a=13.6733(3), b=$ 24.1680(5), $c=14.1445(3) \AA, \beta=109.022(2)^{\circ}, V=4418.9(2) \AA^{3}, Z=2, D_{\mathrm{X}}=1.451 \mathrm{mg} \mathrm{m}^{-3}$, $\lambda(\mathrm{MoK} \alpha)=0.71073 \AA, \mu=0.643 \mathrm{~mm}^{-1}, F(000)=2010, T=130(1) \mathrm{K}$. Data were collected on an Oxford Diffraction Xcalibur Saphir 3 diffractometer (graphite MoK $\alpha$ radiation, $\lambda=$ $0.71073 \AA$ ). The structure was solved with SIR-97 ${ }^{[3]}$ which revealed the non hydrogen atoms of the molecule. After anisotropic refinement, many hydrogen atoms were found with a Fourier Difference. The whole structure was refined with SHELXL97 ${ }^{[4]}$ by the full-matrix least-square techniques (use of $F$ square magnitude; $\mathrm{x}, \mathrm{y}, \mathrm{z}, \beta_{\mathrm{ij}}$ for $\mathrm{Pd}, \mathrm{P}, \mathrm{Cl}, \mathrm{C}$ and $\mathrm{O}$ atoms, $\mathrm{x}, \mathrm{y}, \mathrm{z}$ in riding mode for $\mathrm{H}$ atoms; 1010 variables and 19198 observations with $I>2.0 \sigma(I)$; calc $w=1 /\left[\sigma^{2}\left(F_{\mathrm{O}}^{2}\right)+(0.0815 P)^{2}+0.0000 P\right]$ where $P=\left(F_{\mathrm{O}}^{2}+2 F \mathrm{c}^{2}\right) / 3 . R=0.057, R_{\mathrm{W}}=$ 0.143 and $S_{\mathrm{W}}=0.851, \Delta \rho<3.11 \mathrm{e}^{-3}$. CCDC 758028 contains the supplementary 
crystallographic data for this paper. Crystallographic data can be obtained free of charge at www.ccdc.cam.ac.uk/conts/retrieving.html [or from the Cambridge Crystallographic Data Centre, 12 Union Road, Cambridge CB 11EZ, UK; ax: (internat.) 44-1223/336-033; E-mail: deposit@ccdc.cam.ac.uk].

Crystal structure of $\mathbf{1 3} \cdot \mathbf{C}_{\mathbf{5}} \mathbf{H}_{\mathbf{1 2}}: M \mathrm{r}=1804.99$, orthorhombic, $P 2{ }_{1}{ }_{2}{ }_{2}{ }_{1}, a=17.6384(7), b=$ 19.4659(5), $c=27.2535(8) \AA, V=9357.4(5) \AA^{3}, Z=4, D_{\mathrm{X}}=1.281 \mathrm{mg} \mathrm{m}^{-3}, \lambda(\mathrm{MoK} \alpha)=$ $0.71073 \AA, \mu=0.366 \mathrm{~mm}^{-1}, F(000)=3816, T=100(1) \mathrm{K}$. Data were collected on an Oxford Diffraction Xcalibur Saphir 3 diffractometer (graphite MoK $\alpha$ radiation, $\lambda=0.71073 \AA$ ). The structure was solved with SIR-97 ${ }^{[3]}$ which revealed the non hydrogen atoms of the molecule. After anisotropic refinement, many hydrogen atoms were found with a Fourier Difference. The whole structure was refined with SHELXL97 ${ }^{[4]}$ by the full-matrix least-square techniques (use of $F$ square magnitude; $\mathrm{x}, \mathrm{y}, \mathrm{z}, \beta_{\mathrm{ij}}$ for $\mathrm{Pd}, \mathrm{P}, \mathrm{Cl}, \mathrm{C}$ and $\mathrm{O}$ atoms, $\mathrm{x}, \mathrm{y}, \mathrm{z}$ in riding mode for $\mathrm{H}$ atoms; 1002 variables and 20360 observations with $I>2.0 \sigma(I)$; calc $w=1 /\left[\sigma^{2}\left(F_{\mathrm{o}}{ }^{2}\right)+\right.$ $\left.(0.0600 P)^{2}+0.0000 P\right]$ where $P=\left(F \mathrm{o}^{2}+2 F \mathrm{c}^{2}\right) / 3 . R=0.055, R_{\mathrm{W}}=0.131$ and $S_{\mathrm{W}}=0.770, \Delta \rho$ $<1.608 \mathrm{e}^{-3}$. CCDC 770728 contains the supplementary crystallographic data for this paper. Crystallographic data can be obtained free of charge at www.ccdc.cam.ac.uk/conts/retrieving.html [or from the Cambridge Crystallographic Data Centre, 12 Union Road, Cambridge CB 11EZ, UK; ax: (internat.) 44-1223/336-033; E-mail: deposit@ccdc.cam.ac.uk].

\section{References}

[1] D. Armspach, L. Poorters, D. Matt, B. Benmerad, F. Balegroune, L. Toupet, Org. Biomol. Chem., 2005, 3, 2588-2592.

[2] Hartley, F.R. The Chemistry of Platinum and Palladium; Wiley, New York, 1973.

[3] SIR97, an integrated package of computer programs for the solution and refinement of crystal structures using single crystal data: A. Altomare, M. C. Burla, M. Camalli, G. Cascarano, C. Giacovazzo, A. Guagliardi, A. G. G. Moliterni, G. Polidori, R. Spagna, J. Appl. Crystallogr. 1998, 31, $74-77$.

[4] G. M. Sheldrick, SHELXL-97, Program for Crystal Structure Refinement, University of Göttingen, Göttingen (Germany), 1997. 

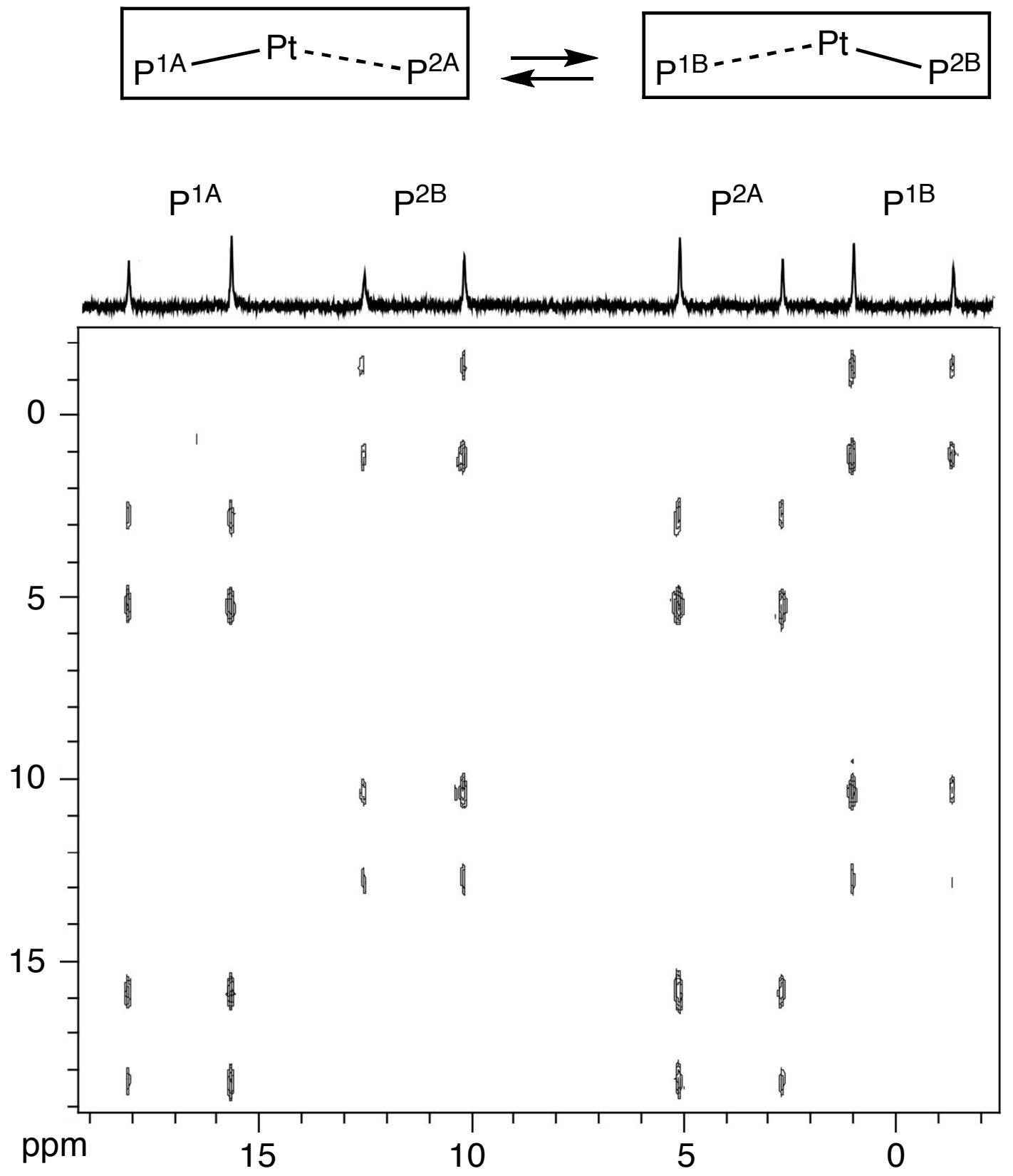

Figure SI-1. ${ }^{31} \mathrm{P}\left\{{ }^{1} \mathrm{H}\right\}$ NMR spectrum and ${ }^{31} \mathrm{P}\left\{{ }^{1} \mathrm{H}\right\}-{ }^{31} \mathrm{P}\left\{{ }^{1} \mathrm{H}\right\}$ COSY $2 \mathrm{D}$ NMR spectrum of complex 10 at $-80^{\circ} \mathrm{C}$ recorded in $\mathrm{CD}_{2} \mathrm{Cl}_{2}$ at $202.5 \mathrm{MHz}$ 

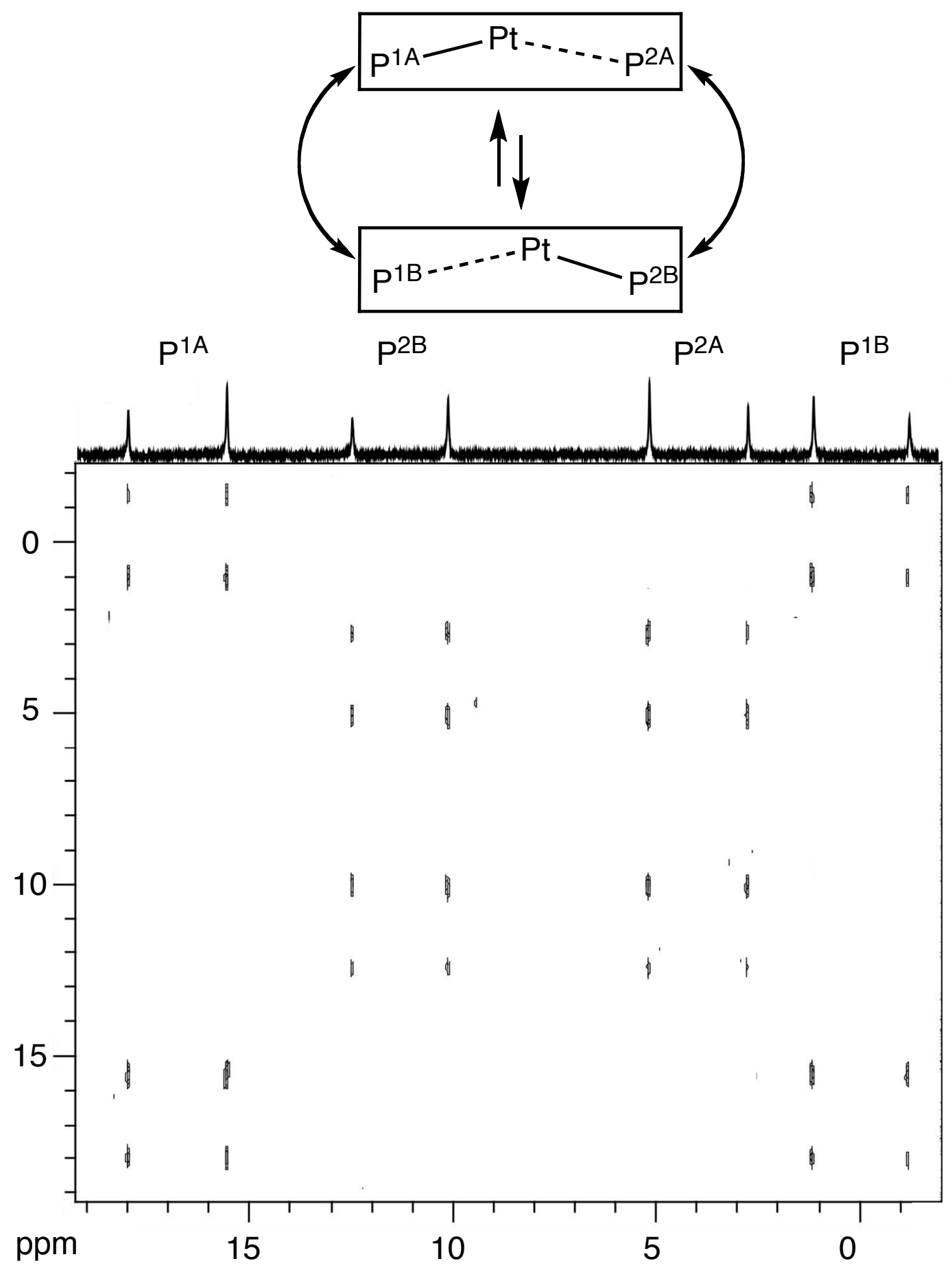

Figure SI-2. ${ }^{31} \mathrm{P}\left\{{ }^{1} \mathrm{H}\right\}$ NMR spectrum and off resonance ${ }^{31} \mathrm{P}\left\{{ }^{1} \mathrm{H}\right\}-{ }^{31} \mathrm{P}\left\{{ }^{1} \mathrm{H}\right\}$ ROESY NMR spectrum of complex 10 at $-60^{\circ} \mathrm{C}$ recorded in $\mathrm{CD}_{2} \mathrm{Cl}_{2}$ at $202.5 \mathrm{MHz}$. 

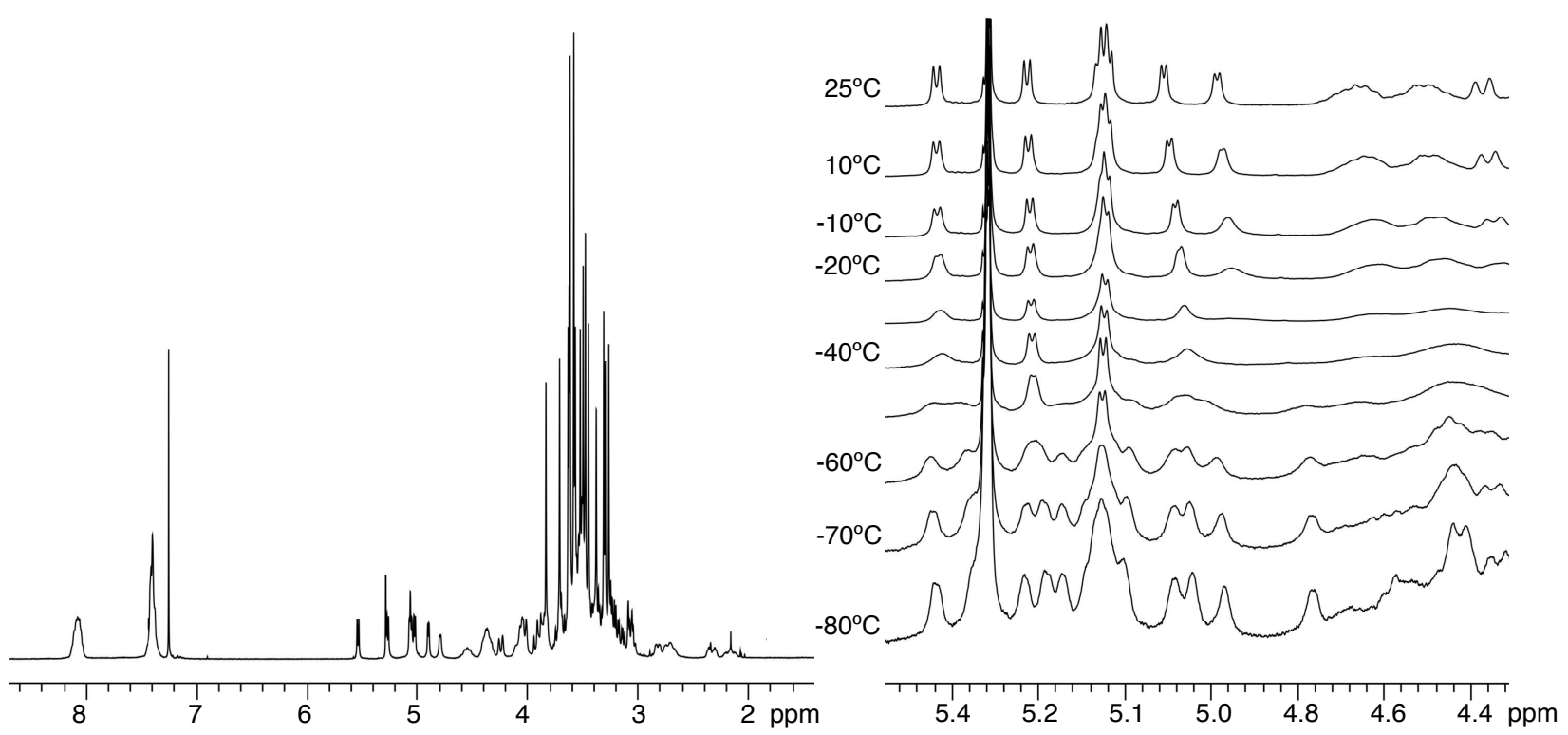

Figure SI-3. ${ }^{1} \mathrm{H}$ NMR spectrum recorded in $\mathrm{CDCl}_{3}$ at $25^{\circ} \mathrm{C}$ at $300.1 \mathrm{MHz}$ (left) and ${ }^{1} \mathrm{H} \mathrm{NMR}$ spectra showing the anomeric protons zone in the range $-80 / 25^{\circ} \mathrm{C}$ recorded in $\mathrm{CD}_{2} \mathrm{Cl}_{2}$ at 500.1 MHz (left) of complex $\mathbf{1 0}$.
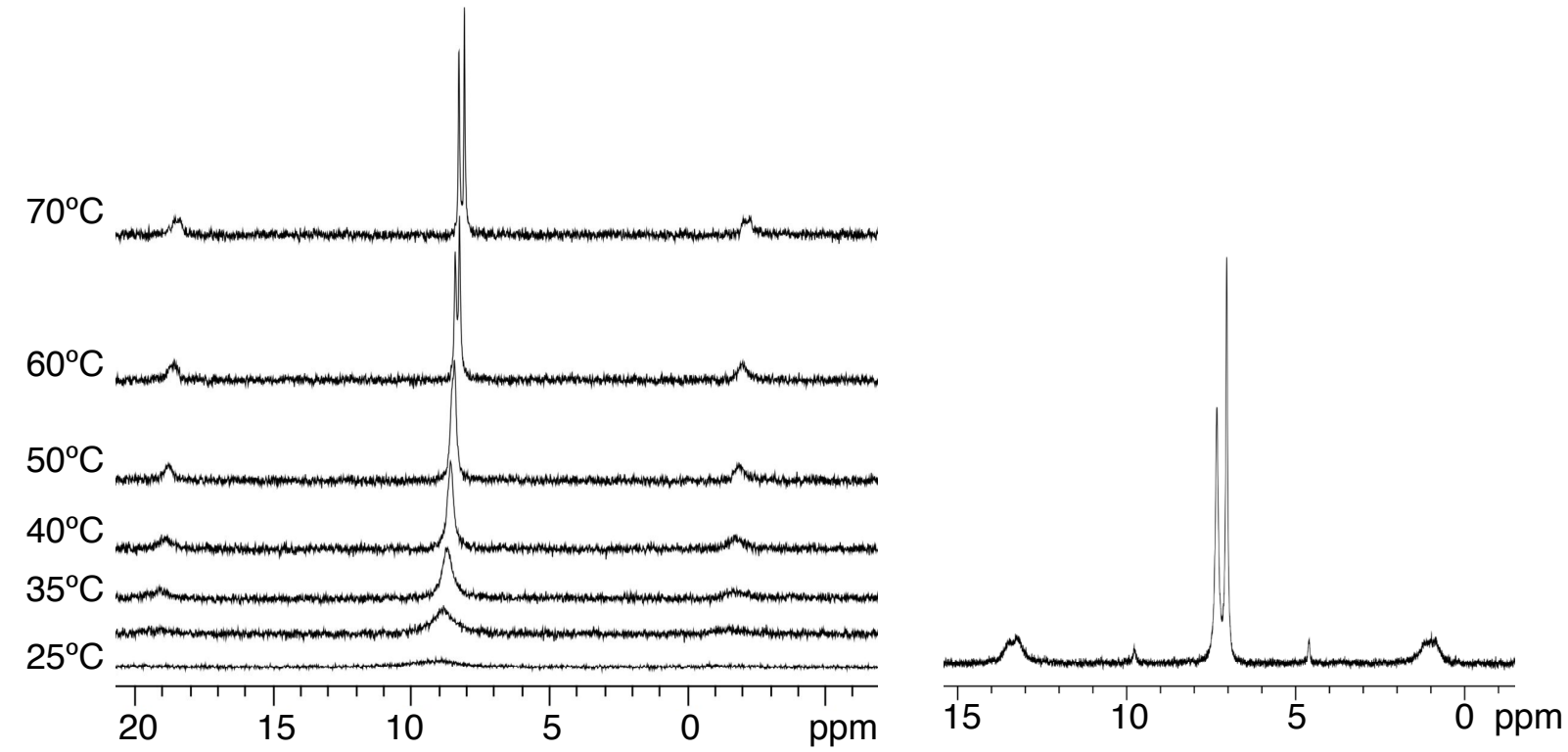

Figure SI-4. ${ }^{31} \mathrm{P}\left\{{ }^{1} \mathrm{H}\right\}$ NMR spectra in the range $25^{\circ} \mathrm{C}-70^{\circ} \mathrm{C}$ at $121.5 \mathrm{MHz}$ (left) and ${ }^{31} \mathrm{P}\left\{{ }^{1} \mathrm{H}\right\}$ NMR spectrum at $80^{\circ} \mathrm{C}$ at $202.5 \mathrm{MHz}$ (right) recorded in $\mathrm{C}_{2} \mathrm{D}_{2} \mathrm{Cl}_{4}$ of complex 10 . 